\title{
The Interactive Role of Emotional Intelligence, Attachment Style, and Resilience in the Prediction of Time Perception in Doctoral Students
}

\author{
Patricia Jean Precin \\ Touro College, New York, USA
}

\begin{abstract}
The perception of time (the use of temporal categories to conceptualize experiences) affects human behavior. Students' time perspective predicts academic outcomes: those with future orientations tend to have better academic outcomes than those with past or present, according to Zimbardo and Boyd's psychology of time model, and may contribute to the $80 \%$ attrition rate of emergent online distance learning programs. However, their model is limited because of the exclusion of certain variables that may influence the development and maintenance of time perspective. Zimbardo and Boyd's model claims that time perspective continually develops and changes but does not explain which variables or aspects of structure and discipline affect time perspective or its plasticity. This nonexperimental quantitative survey study was designed to ascertain whether emotional intelligence, attachment style, and resilience predicted time perspective, since each of these variables has been shown to influence behavior and relate to time perspective. Participants were 93 distance learning doctoral candidates from universities with an online format. Data gathered online from the Zimbardo Time Perception Inventory, Resilience Scale, Psychosocial Maturity Inventory, Revised Adult Attachment Scale, and a demographic questionnaire were analyzed using step-wise logistic regression analyses. Future time perspective was predicted by resilience, independent attachment style, dissertation phase, marital status (married), and gender (male). Present-hedonistic time perspective was predicted by low emotional intelligence, and dissertation phase. Present-fatalistic time perspective was predicted by low emotional intelligence. Past-positive time perspective was predicted by low anxiety attachment scores. Past-negative time perspective was predicted by an anxiety attachment style and high emotional intelligence. Results advanced Zimbaro and Boyd's theory of time perspective by identifying these variables as predictors of time perspective and how they worked together to influence time perspective. The presence of predictors indicated that previous research was incomplete in its explanation of the relationship between time perspectives and human behaviors, such as academic achievement, because of the contribution of emotional intelligence, resilience, and attachment style to time perspective. Results also indicated the need to include these variables in prediction models of attrition rates of online doctoral programs.
\end{abstract}

Keywords: online academic outcomes, distance learning, attrition, all but dissertation, time perspective

Patricia Jean Precin, Ph.D., Psya.D., OTR/L, LP, NCPsyA, FAOTA, licensed practicing psychoanalyst, Assistant Professor of Occupational Therapy, Touro College. 


\section{Chapter 1: Introduction}

Human behavior is explained by many theories, such as behavioral stimulus response (Jessup \& O'Doherty, 2011), cognitive developmental (Pascual-Leone \& Johnson, 2011), psychosocial (Lohr, 2011), self-determination (Silva et al., 2010), psychoanalytic (Schneider, 2010), social learning (Pratt et al., 2010; Wentzel, Battle, Russell, \& Looney, 2010), sociocultural (Swain, Kinnear, \& Steinman, 2011), and ecological systems (Janssen, Holahan, Lee, \& Ostrom, 2010). One very interesting question that has been a focus in the scientific literature (Hamilton, Kives, Micevski, \& Grace, 2003; Keough, Zimbardo, \& Boyd, 1999; Milfont, Andrade, Belo, \& Pessoa, 2008; Zimbardo \& Boyd, 1999, 2008) is how the perception of time (the use of temporal categories or perspectives to conceptualize experiences; Zimbardo \& Boyd, 1999; 2008) may affect human behavior, and in particular, academic achievement.

The phrase "perception of time" usually refers to a broad definition of how time is experienced, which can include objective, subjective, inner-subjective, and multidimensional aspects and has roots of origin in philosophy and science. Many models of time are included in this encompassing definition. However, in this study, the perception of time will take a narrower view and be defined as the use of temporal categories or time perspectives to conceptualize experiences as per Zimbardo and Boyd (1999) and their followers. Time perspective and time perception will be used in this study interchangeably and to refer to the latter definition.

Zimbardo and Boyd (1999; 2008) developed a multi-dimensional model of time perspective that emphasizes the subjective experience of time but also includes objective clock time (Wittmann, 2009) and the inter-subjective experience of time (how being in the presence of others influence the experience of time; Chambon, Droit-Volet, \& Niedenthal, 2008). Time perspective is a learned process by which individuals unconsciously sort personal experiences into temporal perspectives (past, present, future) in order to create meaningful, coherent, and organized events (Zimbardo \& Boyd, 1999; 2008). A balanced time perspective is one without biases towards any particular time perspective (Stolarski, Bitner, \& Zimbardo, 2011) and has been related to success and happiness in life (Zimbardo \& Boyd, 1999; 2008). According to Zimbardo and Boyd's time perspective theory, the present should consist of empirically centered, concrete representations built from psychological, abstract constructions of the past and future expectations. Yet, individuals may over-develop one particular temporal perspective such as the past, present, or future (temporal bias) and have difficulty switching between them when necessary (Zimbardo \& Boyd, 1999; 2008). Individuals with a predominantly past time orientation tend to see the world through their past, which can be viewed as negative (past-negative) or positive (past-positive) (Zimbardo \& Boyd, 1999; 2008). Those with predominantly present time orientations are focused in the present (Zimbardo, 2007). Present-fatalists tend to view the present in a negative way and believe that nothing can be done to improve the future (Zimbardo \& Boyd, 2008). Present-hedonists live for the moment so they tend to seek pleasure and avoid pain (Zimbardo \& Boyd, 1999; 2008). Future time oriented individuals conceptualize the world in terms of their future and often delay gratification in the service of long-term goals (de Bilde, Vansteenkiste, \& Lens, 2011; Zimbardo \& Boyd, 2008).

Most studies on time perception and human behavior (Harber, Zimbardo, \& Boyd, 2003; Horstmanshof \& Zimitat, 2007; Zimbardo \& Boyd, 1999, 2008) including academic achievement (e.g., grade point average, number of hours spent studying, timely completion of assignments and goals) over the last 30 years have been either correlations or investigations of how time perception predicted behavior. Academic achievement has 
been shown to have a positive correlation with a bias for future time orientation and a negative correlation with certain past and present orientations (Boyd \& Zimbardo, 2005; Harber et al., 2003; Zimbardo \& Boyd, 1999). These relationships were linear in that they examined correlations between time perspective and academic achievement instead of considering a multiple variables or models of prediction. Research that has examined how time perspective predicted specific behaviors (academic achievement) may be considered unidirectional in nature because it has omitted the study of predictors of time perception and hence, their possible influence on human behaviors such as academic achievement. Zimbardo and Boyd (1999; 2008) mentioned that education, social class, culture, religion, family modeling (e.g., attachment style, resilience), and other learned factors may determine time perspective biases. Early delayed gratification experiments with children (Zimbardo \& Boyd, 1999) and longitudinal follow-up reports (Zimbardo \& Boyd, 2008) indicated that the predominant type of time perspective an individual acquired in early development lasted into adulthood unless certain learning situations in life or conscious efforts altered the perspective. This is because time perspectives are used to store, encode, and recall experiences; imagine outcomes; set goals; shape expectations; make decisions; feel; and be. In this way, individuals' lives are shaped are by their perception of time, but Zimbardo and Boyd did not provide scientific evidence on what factors shape time perception.

However, variables such as emotional intelligence, psychological resilience, and attachment style have all been related to time perspective (Fortunato \& Furey, 2010; Laghi, D’Alessio, Pallini, \& Baiocco, 2009; Monahan, Steinberg, Cauffman, \& Mulvey, 2010). Emotional intelligence is the ability to identify and regulate emotions and to use emotional awareness of self and others to problem solve and engage in relationships (Côté, DeCelles, McCarthy, Van Kleef, \& Hideg, 2011). Resilience is the process by which an individual interacts (copes) with the environment that includes factors that promote well-being and include risk (Zautra, Hall, \& Murray, 2010). Attachment style is the characteristic manner in which an individual bonds with others (Levy, Ellison, Scott, \& Bernecker, 2011). Age, gender, and income have already been identified as predictors of future time perspective. Future-orientation in older students positively correlated with higher levels of academic performance in terms of dedication to studies, deeper approaches to learning, and applying course material when compared to younger students (Horstmanshof \& Zimitat, 2007). Gender was significantly associated with future-negative time perspective: females had significantly less negative thoughts about the future than males (Peetsma, Schuitema, \& van der Veen, 2012). Income was positively correlated with future orientation (Padawer, Jacobs-Lawson, Hershey, \& Thomas, 2007).

Chapter 1 introduces a nonexperimental quantitative survey study investigating the predicative and interactive role of emotional intelligence, attachment style, and resilience on time perception in doctoral distance learners during a structured (coursework phase) versus a less structured (dissertation phase) of doctoral distance learning. The guiding theoretical pursuit of this research is to ascertain whether emotional intelligence, attachment style, and resilience operate differently to predict time perception in a structured (coursework) versus the less structured (dissertation) phase of doctoral distance learning. If emotional intelligence, attachment style, and/or resilience predict time perspective either individually or in combination, than Zimbardo and Boyd's theory of time perspective could be expanded to include predictors or prediction models of time perspective and not be limited to studies that examined time perception as a predictor of human behavior. The results would indicate that time perspective does not act alone in predicting human behaviors such as academic success, as has been noted in the last 30 years of literature, but that other factors may be shaping the development and sustainability of time perspective. 
The emphasis of this study is not on how emotional intelligence, attachment style, and resilience predict attrition rate directly. Instead, each of these variables has shown significant relationships with time perspective. Mood affected how individuals viewed the future (Klapproth, 2011) and time perspective played a role in structuring emotional experiences (Stolarski et al., 2011). Present-fatalistic time perspective predicted a positive relationship with an avoidant attachment style (Luyckx, Lens, Smits, \& Goossens, 2010). Resilience showed a negative correlation with a past time perspective (Fortunato \& Furey, 2010). Furthermore, there is evidence that emotional intelligence, resilience, and attachment style may work together to influence time perspective. Time perspective mediated the relationship between attachment and resilience (Aronowitz \& Morrison-Beedy, 2004). A strong future orientation mediated the relationship between strong positive social attachments and low levels of aggression (Kruger, Reischl, \& Zimmerman, 2008). Therefore, the predicative nature of emotional intelligence, resilience, and attachment style on time perspective is the focus of this study.

However, since online distance learning doctoral programs have up to an $80 \%$ attrition rate during the dissertation phase of their programs (Nichols \& Levy, 2009), the results of the differences in time perspective and influence of emotional intelligence, attachment style, and resilience on the prediction of time perspective in the two different phases (level of structure) may be integral in the prediction of academic success in online distance learning programs. Literature has shown that certain interventions can help students develop a more future oriented time perspective, and therefore, perform better in school (Ferrari, Nota, \& Soresi, 2012; Horstmanshof \& Zimitat, 2007). Online coursework curriculum could be tailored in such a way as to promote the development of a future time perspective before students entered their dissertation phase in order to prevent attrition from online distance learning doctoral programs.

Chapter 1 begins by presenting the significance of time perception in academia, then introduces four different perspectives on time perception, including Zimbardo and Boyd's (2008) multidimensional model, which will be used in this study. The research problem is described in terms of a limitation of Zimbardo and Boyd's time perspective model, which excludes certain variables such as emotional intelligence, psychological resilience, and attachment style that may influence the development and maintenance of time perspective and, hence, academic achievement. Zimbardo and Boyd's model claims that time perspective continually develops and changes but does not explain which aspects of structure and discipline affect time perspective or its plasticity. Most of the literature describing the relationship of time perspective on academic achievement was performed on traditionally taught college or high school students, not doctoral students enrolled in online distance learning programs. This research problem addresses this gap in the literature. The purpose and design are summarized, followed by the presentation of a theoretical framework for the investigation of predictors of time perception based on Zimbardo and Boyd's model of time perspective. Research questions and their hypotheses are listed, followed by an overview of the nature, methodology, significance, and key operational terms of this study.

\section{Background}

The application of time perception to academia is of particular interest because distance learning programs are becoming more prevalent (Allen \& Seaman, 2011) but have a higher attrition rate than brick and mortar schools (Cockran, 2009; Council of Graduate Schools, 2008) and time perspective has been shown to predict success and failure in academia (Boyd \& Zimbardo, 2005; Harber et al., 2003; Zimbardo \& Boyd, 1999; 
Horstmanshof \& Zimitat, 2007). The growth rate of online learners from 2009 to 2010 was $10 \%$ compared to a $1 \%$ growth rate in classical higher education during the same time frame according to The Sloan Foundation's report (Allen \& Seaman, 2011). However, the attrition rate for all but dissertation distance learners is $70 \%-80 \%$ (Flood, 2002; Nichols \& Levy, 2009), which is higher than the 50\% reported for traditional learners in brick and mortar institutions (Cockran, 2009; Council of Graduate Schools, 2008).

The high attrition rate for distance learners has been attributed in part to poor time management skills (Barbour \& Reeves, 2009; Cockran, 2009; Long, Dubois, \& Faley, 2009; Street, 2010). Time management skills are predicated upon the perception of time (Zimbardo \& Boyd, 1999; 2008). Future oriented students tended to set more long-term academic goals and were more able to delay immediate gratification in order to achieve them than students with a present or past time perspective (Zimbardo \& Boyd, 1999; 2008). College students with a present-hedonistic time perspective tended to be tardy, have poor attendance, sign up for class assignments late, drop out of classes, and have poor grades as compared to their past and future oriented college peers because they chose to satisfy their present desires and were not able to delay immediate gratification (Zimbardo \& Boyd, 1999; 2008).

Not only are time management skills predicated upon time perspective, but time perspective can be influenced by internal states elicited by external stimuli (Levin \& Zakay, 1989). Drive states, moods, and emotions (Droit-Volet \& Meck, 2007; Noulhiane, Mella, Samson, Ragot, \& Pouthas, 2007) have influenced temporal judgments (Taatgen, van Rijn, \& Anderson, 2007) during information processing. The ability to identify and manage emotions, which is part of emotional intelligence, may have an influence on the ability to stave off impulsive desires (giving in to impulsive desires being characteristic of the present-hedonistic time perspective), like going swimming after becoming frustrated with work on a dissertation in order to keep working (the ability to keep working being characteristic of the future time perspective). In this way, emotional intelligence may influence time perspective, which influences the ability to manage time, and therefore, academic outcomes.

In addition, individual characteristics may moderate the impact of internal states (Block, 1990). Two such individual characteristics are psychological resilience and attachment style. A psychologically resilient student may possess the necessary coping skills to manage stressful situations regarding schoolwork, instead of becoming emotionally overwhelmed. In this way, resiliency may influence emotional intelligence and/or resiliency may be acting in consort with emotional intelligence to influence time perspective, which influences the ability to manage time, and therefore, academic outcomes.

Attachment style may also influence and/or work together with emotional intelligence and resilience to influence time perspective. Correlations were noted between attachment style and time perspective (Laghi et al., 2009; Luycks et al., 2010). Future time perspective mediated the relationship between attachment and resilience (Aronowitz \& Morrison-Beedy, 2004). These results may be applied to students. A student with a secure attachment style has the able to form close, trusting bonds with others without experiencing excessive anxiety (Levy et al., 2011). Since there is less anxiety at baseline, securely attached students may be better able to regulate their emotions and may be equipped to handle the pressures associated with completing a dissertation through distance online learning where in-person social contact with professors and mentors does not take place. This type of distance relationship requires trust and consistency in order to develop a working relationship with faculty. The ability to creatively explore educational pursuits is also necessary for completing a dissertation. All of these attributes are characteristic of individuals with secure attachment styles (Berlin, Cassidy, \& Appleyard, 
2008; Bodie et al., 2011). This is in marked contrast to those with an avoidant attachment style, who rarely explore their environments, are emotionally distant, and unconsciously believe their needs will not be met; those with ambivalent or resistant attachment styles who tend to be insecure, angry, and anxious and do not rely on others to meet their needs; and individuals with disorganized attachment styles who are usually angry, passive, non-responsive, depressed, and have few coping strategies to meet their needs (Berlin et al., 2008; Bodie et al., 2011). In this manner, attachment style may influence emotional intelligence and/or be acting in consort with emotional intelligence and/or resilience to influence time perspective, which influences the ability to manage time, and therefore, academic outcomes.

The findings above indicate that time perception may not be acting alone to predict academic achievement in college students as past literature using Zimbardo and Boyd's model of time perception (Zimbardo \& Boyd, 1999; 2008) has proposed. Instead, emotional intelligence, attachment style, and resilience may individually or in combination act together to influence time perspective, which is related to time management, hence attrition in online distance learners.

Therefore, a prediction model is needed in order to ascertain whether emotional intelligence, attachment style, and resilience operate differently to predict time perception in a structured (coursework) versus the less structured (dissertation) phase of doctoral distance learning. A prediction model may address how these variables interact with one another to establish, alter, and sustain time perspective throughout the length of a doctoral distance online learning program. The model may explain questions that Zimbardo and Boyd's model of time perception could not previously account for. One such question is, why does the structure of school help some individuals succeed while it hinders others? Specifically, why does school help discipline some students (e.g., by helping them move from a present-hedonistic to a more future orientation) but not all students?

This current study examined whether or not emotional intelligence, attachment style, and resilience act individually or through an interactive model to predict time perspective in doctoral distance online learners. It also examined how the prediction model may differ given different levels of structure: coursework phase (higher level of structure) and dissertation phase (less structure) of the program.

Zimbardo and Boyd's (2008) model of time perspective was used for this study to explore the role of time perception in terms of five cross-culturally sensitive (Milfont, Andrade, Bela, \& Pessoa, 2008) time perspectives (i.e., past-negative, past-positive, present-fatalistic, present-hedonistic, and future, which is defined in the Definition of Key Terms). These time perspectives are cross-culturally sensitive in that they define constructs for which validity has been established for different cultures and in multiple countries (Brazil (Milfont et al., 2008), Spain (Díaz-Morales, 2006), Italy (D’Alessio, Guarino, de Pascalis, \& Zimbardo, 2003), Mexico (Corral-Verdugo, Fraijo-Sing, \& Pinheiro, 2006), France (Apostolidis \& Fieulaine, 2004), and others). Zimbardo and Boyd (2008) theorized that an individual's time perspective develops early in life through the influence of many factors (e.g., culture, personal experiences, religion, social models, geography, climate, school, family, peers, work) that last into adulthood unless certain learning situations or conscious efforts alter the perspective, such as resilience, emotional maturity, and attachment style, which are not addressed in the theory. Age, income, and gender also predicted time perspective (Padawer et al., 2007).

\section{Statement of the Problem}

Zimbardo and Boyd's (1999; 2008) linear unidirectional application of time perspective to academic achievement is limited because of the exclusion of emotional intelligence, resilience, and attachment style that 
may influence the development and maintenance of time perspective and hence, academic achievement. Zimbardo and Boyd's (2008) model of time perspective over the past 30 years has focused mostly on the identification of correlations between time perspective and different human behaviors or studies that identified different human behaviors that were predicted by time perception. Their model does not investigate predictors of time perspective, although it states that certain environmental, cultural, geographic, and internal processes can help shape time perspective. Their model does not state how these variables may contribute to the development of time perspective. Their model reported strong positive correlations between future time orientation and academic success (Zimbardo \& Boyd, 1999), but it does not address the question of why the structure of school helps some students succeed while it hinders others. Certain students can learn to thrive under the structure of an academic setting (move from a past-negative or present-hedonistic time perspective bias to a future orientation) and others cannot. It does not address the phenomena of flexibility of time perspective that some individuals possess and others do not, remaining fixed in a particular bias instead. Without the consideration of the contribution of emotional intelligence, resilience, and attachment style to the development and maintenance of time perspective, studies that have reported that time perspective predicted academic success may be incomplete. Flexibility between time perspectives cannot be addressed. The potential for interventions to develop future time perspectives in students entering the dissertation phase of their programs may not be considered. And, finally, predictions of success or failure in online distance learning doctoral programs cannot be accurately determined.

In addition, Zimbaro and Boyd's theory claims that time perception continually develops and changes but does not explain which aspects of structure and discipline affect time perspective or its plasticity. Students choose online distance learning programs because they offer a flexible schedule (Parsad \& Lewis, 2008). The freedom to study at convenient times sounds desirable, yet more students drop out of online distance learning programs than do those enrolled in classical programs (Cockran, 2009) due to an inability to manage time or anticipate the amount of discipline necessary to complete assignments without the structure of deadlines (Cockran, 2009; Street, 2010). Other reasons related to time may exist that make it difficult for students to complete online distance doctoral programs. Students may not be able to balance work, leisure, school work, responsibilities in other roles, and sleep, necessary for mental and physical health (Christiansen \& Matuska, 2006). Another may be related to how students experience their daily activities. If most of their activities during a day lead to feelings of accomplishment, joy, and satisfaction, then students may be more motivated and able to continue in the program. If they feel anxious, angry, depressed, or bad about themselves during their daily experiences, they may have difficulty continuing their educational goals. Certain students require a high degree of structure in order to be productive in academic pursuits, while others feel trapped and stifled with too much structure and too many rules. Coursework tends to be more structured than the dissertation phase, so some students may do better during the coursework phase versus the dissertation phase or vice versa. Other factors such as emotional intelligence, resilience, and attachment styles may be influencing time perspective.

Given these gaps in Zimbardo and Boyd's theory of time perspective (1999; 2008), resilience, emotional maturity, and attachment style were examined for their influence on time perception because of their stated relationships to behavior and time perception in the literature. Resilience, emotional maturity, and attachment style affected individual choices (Beauvais, Brady, O'Shea, \& Griffin, 2011; De La Rosa, Dillon, Rojas, Schwartz, \& Duan, 2010; Yoo, Slack, \& Holl, 2010) and correlated with time perspective (Fortunato \& Furey, 2010; Laghi et al., 2009; Monahan et al., 2010). Time perception was a mediating variable for the relationship 
between: (1) resilience and academic outcomes (Kruger et al., 2008); (2) belief in a just world and resilience (Xie, Liu, \& Gan, 2010); and (3) attachment and resilience (Kruger et al., 2008). Age, gender, and income have predicted future time perspective (Padawer et al., 2007).

The manner in which resilience, emotional maturity, and attachment style may predict time perception is unknown. Results advance Zimbaro and Boyd's theory of time perspective by identifying predictors and prediction models of time perspective. The presence of predictors indicate that previous research was incomplete in its explanation of the relationship between time perspectives and human behaviors, such as academic achievement, because of the contribution of emotional intelligence, resilience, and attachment style to time perspective. Results also indicate the need to include these variables in prediction models of attrition rates of online doctoral programs. The guiding theoretical pursuit of this research was to ascertain whether emotional intelligence, attachment style, and resilience operate differently to predict time perception in a structured (coursework) versus the less structured (dissertation) phase of doctoral distance learning.

\section{Purpose of the Study}

The purpose of this nonexperimental quantitative survey study was two-fold. First, it was used to identify what predictive role emotional intelligence, attachment style, and resilience (predictor variables) had on time perception (criterion variable) in doctoral distance learners. Second, the study was used to ascertain how emotional intelligence, attachment style, and resilience (predictor variables) interacted to predict time perception (criterion variable). Participants were 93 distance learning doctoral candidates from universities with an online format, 51 participants were in the coursework phase, 38 participants were in the dissertation phase of their program (level of structured time as the predictor variable) and four did not indicate which phase of the program they were in. The needed sample size was determined by G*Power 3.1.5 (Faul, Erdfelder, Buchner, \& Lang, 2009) using the $F$-test for linear multiple regression: fixed model, $R$-squared deviation zero with all input parameters: alpha $=0.05$, power $=0.80$, effect size $=0.15$, and number of predictors $=3$. Time perception was measured by the Zimbardo Time Perception Inventory (ZTPI) (Zimbardo \& Boyd, 1999; see Appendix A). Resilience will be measured by the Resilience Scale (RS) (Wagnild \& Young, 2009; see Appendix B). Emotional intelligence was measured by the Psychosocial Maturity Inventory (PMI) (Greenberger, Josselson, Knerr, \& Knerr, 1974; see Appendix C). Attachment style was measured by Collins and Read's (1990) Revised Adult Attachment Scale (RAAS; see Appendix D). Demographic data was collected using the Demographic Questionnaire (see Appendix E) developed by this researcher. Multiple linear regression analysis was conducted to evaluate how well emotional intelligence, resilience, and attachment style predicted time perspective in each phase of learning. Multiple linear regression analysis was performed to evaluate how attachment style, resilience, and emotional intelligence interacted to predict time perspective in each phase of learning. The results provided clarification to Zimbardo and Boyd's model of time (1999; 2008) by uncovering the predictive role of attachment style, emotional maturity, and resilience on time perception during a structured phase (coursework phase) and the less structured phase (dissertation phase) of doctoral distance learning.

\section{Theoretical Framework}

Zimbardo and Boyd's psychology of time model $(1999 ; 2008)$ is one of the models that have been used to examine time attitudes as they relate to academic success in traditionally taught high school and college students. To date, of the literature reviewed, nothing has been published on its use with distance learners or 
doctoral students, yet there is the potential for a direct application. In this section, Zimbardo and Boyd's psychology of time model and its development is explained.

Zimbardo and Boyd's psychology of time model. According to Zimbardo and Boyd (1999; 2008), time perception is the learned process by which individuals unconsciously sort personal experiences into temporal categories or time zones in order to create meaningful, coherent, and organized events. Zimbardo and Boyd (1999) developed the Zimbardo Time Perspective Inventory, to measure individuals' orientations to the past, present, and future. These time perspectives were found to include past-negative, past-positive, present-fatalistic, present-hedonistic, and future categories as determined by exploratory and confirmatory factor analyses (Zimbardo \& Boyd, 1999), verified cross-culturally (Milfont et al., 2008), and supported in recent literature (Worrel \& Mello, 2007). Individuals with a predominantly past time orientation tend to see the world through their past, which can be viewed as either negative (past-negative) or positive (past-positive) (Harber et al., 2003; Zimbardo \& Boyd, 1999, 2008). Those with predominantly present time orientations are focused in the present. Present-fatalists tend to view the present in a negative way and believe that nothing can be done to improve the future (Harber et al., 2003; Zimbardo \& Boyd, 1999, 2008). Present hedonists live for the moment so they tend to seek pleasure and avoid pain. Future time oriented individuals think of the world in terms of their future and often delay gratification in the service of long-term goals (de Bilde et al., 2011; de Volder \& Lens, 1982; Harber et al., 2003; Zimbardo \& Boyd, 1999, 2008). Early experiments involving children and delayed gratification (Zimbardo \& Boyd, 1999) and follow-up longitudinal reports (Zimbardo \& Boyd, 2008) indicated that the predominant type of time perspective an individual acquired in early development lasted into adulthood unless certain learning situations in life or conscious efforts altered the perspective. Zimbardo and Boyd (1999; 2008) identified interventions that they found clinically useful to alter maladaptive time perspectives to promote happier more functional lives, as past-negative and present-fatalistic perceptions were associated with depression and a lower level of functioning.

Zimbardo and Boyd's psychology of time (2008) explains a subjective view of how people's attitudes towards time or time perspective affect their behavior and therefore, impact their lives. Over the last 30 years, research has shown that each of Zimbardo and Boyd's time perspectives has been associated with a variety of human behaviors because time perspectives have been reported to influence judgments, actions, and decision making (Nuttin \& Lens, 1985; Zimbardo \& Boyd, 1999). A past-negative perspective correlated positively with aggression, depression, and anxiety (Zimbardo \& Boyd, 1999); pathological gambling and psychiatric conditions (Hodgins \& Engel, 2002); and exercise (Hamilton et al., 2003). Negative correlations were reported for past-negative and learners' level of satisfaction with their learning institutions (Milfont et al., 2008). Past-positive orientation positively correlated with self-esteem (Zimbardo \& Boyd, 1999) and health, religiosity, and nutrition (Hamilton et al., 2003) while negatively with anxiety, aggression, and depression (Zimbardo \& Boyd, 1999). Present-hedonistic orientation showed a positive association with social relations and health (Hamilton et al., 2003); drug and alcohol misuse (Keough et al., 1999); need for high stimulation and novel experiences and weak ego strength (Zimbardo \& Boyd, 1999); and dangerous driving (Zimbardo, Keough, \& Boyd, 1997). Associations in the negative direction were reported between present-hedonistic and water conservation (Corral-Verdugo et al., 2006) and spirituality, consistency, and use of a watch to track time (Zimbardo \& Boyd, 1999). Present-fatalistic predicted aggression, depression, and anxiety (Zimbardo \& Boyd, 1999 ) and was positively associated with exercise (Hamilton et al., 2003). Present-fatalistic orientation was negatively associated with learners' satisfaction with their institute of study (Horstmanshof \& Zimitat, 2007); 
importance of future consequences (Zimbardo \& Boyd, 1999); and behavior indicative of concern for the environment (Corral-Verdugo et al., 2006). Future orientation correlated positively with positive affects, coping skills, resilience, well-being, and perceived control over life situations (Wills, Sandy, \& Yaeger, 2001); and behavior that attempted to protect the environment (Corral-Verdugo et al., 2006). Negative correlations were reported for future orientation and depression, anxiety, and the need for high stimulation (Zimbardo \& Boyd, 1999); dangerous driving (Zimbardo et al., 1997); age (Hamilon et al., 2003); and substance misuse (Keough et al., 1999).

Academic achievement has been studied extensively as outlined below and is most pertinent to the currently proposed dissertation. Future oriented time perspective was consistently related to positive academic outcomes across many studies. Zimbardo, Boyd, and co-workers found that people who were the most academically successful tended to have a future orientation, allowing them to see ahead, delay gratification, set goals, and manage their time, whereas present oriented individuals had the highest college attrition rate (Boyd \& Zimbardo, 2005; de Volder \& Lens, 1982). Individuals who scored high in future orientation were more punctual for class, apt to complete homework before due dates, inclined to begin assignments on time, and skilled in time management than their classmates (Harber et al., 2003). The same study reported significant gender differences; future oriented females began their assignments before their male counterparts. Future oriented college students also tended to utilize successful learning strategies such as reproductive and deep-level cognitive processing more often than students with other time orientations (Horstmanshof \& Zimitat, 2007). In addition, persistence (Peetsma, 1994; Zaleski, 1987), level of engagement (Peetsma, 1994), satisfaction (Zaleski, 1987), number of hours spent in school work (Peetsma, 1994) and grade point average (Zimbardo \& Boyd, 1999) were related to future orientation among college students. Future orientation was also associated with academic achievement in high school learners (Anderman, Anderman, \& Griesinger, 1999; de Volder \& Lense, 1982; Groth, 1973; Honora, 2002; Shell \& Husman, 2001). Conversely, there was a negative correlation between past-negative and students' report of satisfaction with their educational institutions (Horstmanshof \& Zimitat, 2007). Students oriented to the present were late signing up for, initiating, and completing assignments, so they tended to receive more incompletes and poorer grades, were assigned to special education classes more often, and dropped out of school more frequently than their counterparts (Harber et al., 2003). The poorest grades were characteristic of fatalists then hedonists.

In addition to predicting specific human behaviors, Zimbardo and Boyd's time perspectives have been examined in combination to determine an overall time profile of successful and unsuccessful individuals in the United States. Success in work, social relations, quality of life, and happiness were associated with a time perspective profile of: high in past-positive, moderately high in future, moderately high in present-hedonistic, low in past-negative, and low in present-fatalistic (Boniwell \& Zimbardo, 2003, 2004; Holman \& Silver, 1998; Zimbardo \& Boyd, 2008) and the flexibility to switch between different time perspectives as needed (Boyd \& Zimbardo, 2005). Individuals scoring high in present-fatalist and past-negative time perspectives tended to have poor social skills and negative affects (Boniwell \& Zimbardo, 2003, 2004; Holman \& Silver, 1998; Holman \& Zimbardo, 2009).

Development of Zimbardo and Boyd's psychology of time model. The study of the importance of time has been an ongoing controversy. William James, the father of psychology, devoted a chapter on time perception in The Principles of Psychology (1950/1890); yet later behaviorists deemphasized the importance of consciousness and research on time (Skinner, 1987; Watson, 1913). Albert Einstein showed that time was 
relative (1931). Social psychologists believed that time was a social construct, and, therefore, also relative in nature (Moscovici, 1984). However, an exhaustive discourse on the concept of time is not necessary because the development of Zimbardo and Boyd's psychology of time theory $(1999 ; 2008)$ can be traced back to the seminal German philosopher, Immanuel Kant (1724-1804) and his followers.

Kant thought that the conception of time was innate and that it affected the way individuals viewed the world (1965/1781). Kant's idea of time was expanded by existential psychologists and philosophers (Heidegger, 1927; Husserl, 1964). Kurt Lewin, a Gestalt psychologist who started social psychology, was influenced by Kant's thinking about time. Rejecting the narrow focus of the behavioral psychologists with regards to time and embracing, instead, an existential view, Lewin developed the life space model, which purported that the past and future not only affected the way individuals viewed the world but influenced present behavior (1951). He believed time perspective was a function of all of an individual's views of his or her past and future as experienced in the present moment. This holistic view of time went against the Western experience of and belief in time as linear. It more closely resembled Eastern philosophy and the Zen nature of time as circular (Orenstein, 1975). Nuttin was a follower of Lewin's time-filled space model and expanded it to include the idea that the past and future affected present behavior on a cognitive level (1964). However, Nuttin deemphasized the effect of the past on present behavior in favor of the idea that motivational space resided in the future. Cognition was also linked with time in the self-efficacy theory of Bandura (1997), which stated that behavioral self-regulation was influenced by experiences from the past, thoughts about future options, and judgments that occurred in the present moment. Shortly after, Carstensen, Isaacowitz, and Charles (1999) suggested that time perception directly influenced goal setting and goal attainment with cognition, motivation, and emotion also playing a role.

Zimbardo and Boyd's work began in the early 1970s because they were curious about their personal relationship to time and its effect on their behavior. They induced a present time perspective in college students who were future time oriented and reported significant alterations in their behavior, feelings, and thoughts (Zimbardo, Marshall, \& Maslach, 1971). Shortly after, they were influenced by Darley and Batson's Good Samaritan study on helping behavior and time press (1973). This study showed that seminary students at Princeton who should be predisposed to want to help individuals in need, under the pressure of time, put their needs before the needs of another who required medical attention. Yet, their classmates, not under a time constraint, took the necessary actions to help the victim in distress before meeting their need to present a speech for a grade. This difference in behavior was said to be caused by the manipulation of time and suggested that time perspective alters behavior. Zimbardo and Boyd then became interested in furthering the exploration of the relationship of time to behavior but found a lack of empirical tools to assess this controversial and complicated topic. The tools that did exist measured time perspective on one-dimension only (i.e., sensation-seeking (present time orientation) (Zuckerman, 1994; Zuckerman, Eysenck, \& Eysenck, 1978) and future orientation (Strathman, Gleicher, Boninger, \& Edwards, 1994; Zaleski, 1996)). After 30 years of their own research, Zimbardo and Boyd believed that time perception was multidimensional and published a valid and reliable measurement tool to quantitatively assess the subjective multidimensionality of time perception, the Zimbardo Time Perspective Inventory (Zimbardo \& Boyd, 1999; see Appendix A). The five factors of time perception of the Zimbardo Time Perspective Inventory: past-negative, past-positive, present-fatalistic, present-hedonistic, and future orientations are orthogonal in that a subject could score high or low on all five (versus obtaining a percentage on each that would total 100 percent). 
This instrument integrated past time perspective research, provided a means to further examine time perspective's relationship to behavior in a standardized manner, and led to the maturation of Zimbardo and Boyd's psychology of time theory. The theory incorporated: Einstein's theory of relativity, social psychologists' belief that time was a social construct, Kant's theory that time affected one's view of the world and was an innate ability (but one, according to Zimbardo and Boyd that could be changed), the Lewinian expansion of Kant's theory that the perception of time directly influenced behavior, and the addition of cognition (Nuttin) and self-efficacy (Bandura) to the construct of time perception and the resultant effect on behavior.

Given the influence of these theoretical views and their own and other current research, Zimbardo and Boyd's current psychology of time theory includes the following concepts as summarized in The Time Paradox, a culmination of Zimbardo and Boyd's research (Zimbardo \& Boyd, 2008). Environmental aspects (e.g., the pace of life) are internalized, become part of the culture, and exert an often unconscious effect on people's feelings, thoughts, and behaviors. Time perspectives are learned and subjective. The more awareness an individual has about his or her particular perspective of time, the more able he or she will be to adjust it for the better. Individuals partition ongoing events into temporal-cognitive frames that render meaning, coherence, and order to experiences. These frames can be repetitive, unique, cyclical, or non-recurring linear events (Hall, 1983). Time perspectives are used to store, encode, and recall experiences; imagine outcomes; set goals; shape expectations; make decisions; feel, and be. Hence, the way individuals live is shaped by their perception of time. Optimally, the present consists of empirically centered, concrete representations built from psychological, abstract constructions of the past and future expectations. Yet, individuals may over-develop one particular time perspective such as the past, present, or future (temporal bias) and have difficulty switching between them when necessary. Education, social class, culture, religion, family modeling, and other learned factors may determine this overreliance. If habitual, a dispositional style can arise that is predicative of many behaviors as noted above. This is in contrast to a balanced time orientation that can change according to circumstance.

Zimbardo and Boyd's (1999; 2008) psychology of time model began by examining correlations and associations between different time perspectives and behaviors in the areas of health and risk taking among college students in the United States. It predicted success, happiness, depression, anxiety, conscientiousness, creativity, energy, friendliness, exercise, impulse control, ego control, temper, self-esteem, shyness, reward-dependence, gambling, emotional stability, and other behavioral characteristics (Boyd \& Zimbardo, 2005; Zimbardo \& Boyd, 2008). The theory advanced cross-culturally to 32 nations to date (Brazil (Milfont et al., 2008), Spain (Díaz-Morales, 2006), Italy (D’Alessio et al., 2003), Mexico (Corral-Verdugo et al., 2006), France (Apostolidis \& Fieulaine, 2004), and others) and cross-socioeconomically (Lamm, Schmitdt, \& Trommsdorff, 1976).

Resent research using Zimbardo and Boyd's model of time perception included time perspective profiles of juvenile delinquents (Oyserman \& Markus, 1990a; 1990b) inmates (Haney \& Zimbardo, 2009), politicians and businessmen (Zimbardo, 2007), and individuals diagnosed with posttraumatic stress disorder and other psychiatric disturbances (Zimbardo \& Boyd, 2008). Since Zimbardo and Boyd were also clinicians, they developed and are developing therapeutic interventions to switch maladaptive time perspectives to more adaptive ones depending on the individual's needs in context (Zimbardo \& Boyd, 2008). Documentation of these therapeutic outcomes is needed.

Future directions for the Zimbardo and Boyd psychology of time model include examining each time perspective's developmental history and how each are strengthened, weakened, learned, and maintained over 
time. Research investigating the difference between individuals with balanced time perspectives and those with biases may be helpful. And, an exploration of the use of time perspective profiles in the analysis of group behavior such as war (and the climate of war) and technological changes in addition to categorical analysis used by the Zimbardo Time Perspective Inventory (Zimbardo \& Boyd, 1999). Specific to this study is the identification of predictors of time perception.

\section{Research Questions}

The following research questions were used to evaluate the purpose of this study. The research questions were used to examine the strength of prediction of attachment style, resilience, and emotional intelligence on time perception. The questions were also used to examine how attachment style, resilience, and emotional intelligence interact to predict time perception in doctoral distant learners in the coursework and dissertation phase of their programs. Corresponding null hypotheses and alternative hypotheses are given.

Q1: Does resilience predict time perspective in coursework and dissertation phases of a doctoral distance learning program?

Q2: Does attachment style predict time perspective in coursework and dissertation phases of a doctoral distance learning program?

Q3: Does emotional intelligence predict time perspective in coursework and dissertation phases of a doctoral distance learning program?

Q4: How will resilience, attachment style, and emotional intelligence interact to predict time perspective during the coursework and dissertation phases of a doctoral distance learning program?

\section{Hypotheses}

$\mathrm{H} 1_{0}$ : Resilience will not significantly predict time perspective during the coursework and dissertation phases of a doctoral distance learning program.

H1a: Resilience will significantly predict time perspective during the coursework and dissertation phases of a doctoral distance learning program.

$\mathrm{H} 2_{0}$ : Attachment style will not significantly predict time perspective during the coursework and dissertation phases of a doctoral distance learning program.

$\mathrm{H} 2 \mathrm{a}$ : Attachment style will significantly predict time perspective during the coursework and dissertation phases of a doctoral distance learning program.

$\mathrm{H}_{0}$ : Emotional intelligence will not significantly predict time perspective during the coursework and dissertation phases of a doctoral distance learning program.

H3a: Emotional intelligence will significantly predict time perspective during the coursework and dissertation phases of a doctoral distance learning program.

$\mathrm{H}_{0}$ : Resilience, attachment style, and emotional intelligence will not significantly interact to predict time perspective during the coursework and dissertation phases of a doctoral distance learning program.

H4a: Resilience, attachment style, and emotional intelligence will significantly interact to predict time perspective during the coursework and dissertation phases of a doctoral distance learning program.

\section{Nature of the Study}

In this nonexperimental quantitative study, a cross-sectional survey design was used to identify what predicative role emotional intelligence, attachment style, and resilience have on time perception in doctoral distance learners. The study was used to ascertain how emotional intelligence, attachment style, and resilience 
interacted to predict time perception. The design of this study was based on others in the literature that used a nonexperiemental, quantitative, cross-sectional, online survey design to identify relationships between time perspective and human behavior (Harbor et al., 2003; Horstmanshof \& Zimitat, 2007; Milfont et al., 2008; Zimbardo, 2007; Zimbardo \& Boyd, 1999) and time perspective predictors of human behavior (Barber, Munz, Bagsby, \& Grawitch, 2009; Dunkel \& Weber, 2010; Xie et al., 2010; Zimbardo \& Boyd, 1999). Time perspective was evaluated through the use of a multidimensional time perspective inventory that measures individuals' orientation to the past, present, and future. This inventory, the ZTPI, has been used throughout the literature in seminal studies that examined the relationship between time perception and various human behaviors (Apostolidis \& Fieulaine, 2004; Corral-Verdugo et al., 2006; D’Alessio et al., 2003; Díaz-Morales, 2006; Harbor et al., 2003; Horstmanshof \& Zimitat, 2007; Milfont et al., 2008; Zimbardo \& Boyd, 1999). In terms of the past dimension of time perspective, the following constructs were evaluated:

(1) Past-positive, as measured by the ZTPI (Zimbardo \& Boyd, 1999; see Appendix A); and

(2) Past-negative, as measured by the ZTPI (Zimbardo \& Boyd, 1999; see Appendix A).

In terms of the present dimension of time perspective, the following constructs were evaluated:

(1) Present-fatalistic, as measured by the ZTPI (Zimbardo \& Boyd, 1999; see Appendix A); and

(2) Present-hedonistic, as measured by the ZTPI (Zimbardo \& Boyd, 1999; see Appendix A).

In terms of the future dimension of time perspective, the following constructs were evaluated:

Future, as measured by the ZTPI (Zimbardo \& Boyd, 1999; see Appendix A).

Emotional intelligence markers were used to indicate the ability to identify and regulate emotions, ability to use emotional awareness of self and others to problem solve and engage in relationships, and sense of personal responsibility. The following constructs of emotional intelligence were measured using the PMI (Greenberger, Josselson, Knerr, \& Knerr, 1974; see Appendix C):

(1) self-reliance;

(2) work orientation; and

(3) identity.

Attachment style markers were used to indicate the manner in which participants bonded with others. The following constructs of attachment style were measured using the RAAS (Collins \& Read, 1990; see Appendix D):

(1) depend (the ability to depend on others);

(2) anxiety (the fear of abandonment); and

(3) close (the ability to become and stay close with others).

Resilience markers were used to indicate the manner in which participants interacted (coped) with their environment. The following constructs of resilience were measured using the RS (Wagnild \& Young, 2009; see Appendix B):

(1) meaningful life;

(2) perseverance;

(3) self reliance;

(4) equanimity; and

(5) existential aloneness.

Gender, income, and age were measured on the basis of demographic information provided by participants using a demographic survey constructed for the purpose of this study (see Appendix E). The relationship between time perspective and gender (Harbor et al., 2003; Peetsma et al., 2010), income (Zimbardo \& Boyd, 
1999), and age (Ferrari et al., 2012; Horstmanshof \& Zimitat, 2007) has been examined in the literature using demographic surveys.

The use of the Internet afforded the opportunity to recruit participants of diverse ethnicity from locations throughout the United States into a nonprobability, purposive sample. Inclusion criteria included distance learners currently enrolled in an online doctoral program in the United States. A total of 93 distance learners were used. Fifty-one participants were in the coursework phase, 38 participants were in the dissertation phase of their programs, and four did not indicate which program they were in.

Participants completed self-report questionnaires for quantitatively measuring time perception, emotional intelligence, attachment style, and resilience. Level of imposed structure in the doctoral program (coursework phase-high level of structure; dissertation phase-low level of structure), emotional intelligence, attachment style, and resilience constituted the predictor variables. Demographics gathered included age, gender, and income. Time perception was the criterion variable.

Predictor variables. To obtain values for the predictor variables, participants were asked to complete a survey containing the following three published, standardized, reliable, and validated instruments:

The Psychosocial Maturity Inventory (Greenberger et al., 1974; see Appendix C). The PMI is a 30-item reverse-scored scale, which evaluates personal responsibility and has 0.89 in reliability and an adequate fit to baseline data $(\mathrm{NFI}=0.82, \mathrm{CFI}=0.87, \mathrm{RMSEA}=0.04)$. A revision of the PMI was used to measure emotional intelligence. Eighteen of the 30 items were selected for use with this study. The eighteen scale items maintain their reliability and validity when used without the other items (Greenberger et al., 1974).

The Revised Adult Attachment Scale (Collins \& Read, 1990; see Appendix D). The RAAS was used to measure participants' attachment style quantitatively: Dependent, Anxious, and Close. The RAAS has internal consistency of 0.75 for Depend, 0.72 for Anxiety, and 0.69 for Close items and test-retest reliability of $0.71,0.52$, and 0.68 respectively as measured by Cronbach's alpha coefficient and reported by Collins and Read. The RAAS has been validated and used to study the relationship between attachment and trust, self-esteem, love, child-parent relationships, and social self-perception.

The Resilience Scale (Wagnild \& Young, 2009; see Appendix B). The RS, a 14-item assessment, was used to measure resilience in five characteristics of resilience: existential aloneness, self-reliance, perseverance, purposeful life, and equanimity; the scale also yields a total score. The RS was modified from the original 50-item Resilience Scale and has high internal consistency (Nishi, Uehara, Kondo, \& Matsuoka, 2010), a test-retest reliability of 0.91 (Wagnild \& Young, 2009), and low concurrent validity (Nishi et al., 2010).

A question regarding participants' current phase of their doctoral program (coursework versus dissertation) was answered on the demographic survey (see Appendix E) to ascertain the level of structure.

Demographics. To identify the demographics of age, income, educational level, school, marital status, ethnicity, learning environment, and gender, participants completed the rest of the demographic survey constructed for this study (see Appendix E).

Criterion variable. The criterion variable of time perception was measured by asking participants to complete the following published, standardized, reliable, and validated instrument:

The Zimbardo Time Perspective Inventory (Zimbardo \& Boyd, 1999; Appendix A). The ZTPI was used to quantitatively measure the type of perspective each participant had about time on five different time perspectives: past-negative, past-positive, present-fatalistic, present-hedonistic, and future. Test-retest reliability coefficients have ranged from 0.70 to 0.80 in college psychology majors over a four-week interval and 
structural validity was established using exploratory and confirmatory factor analyses (Zimbardo \& Boyd, 1999).

Analyses. SPSS Statistics Gradpack version 19.0 (2011) statistical software was used for all data analysis. A correlation matrix was used to examine whether the quantitative variables correlated. A multiple linear regression analysis across all participants was performed using the Enter Method. First, tests of the difference between regression coefficients were performed (Faul et al., 2009) and if they were significantly different, reliabilities were estimated for the different categories of gender and slopes dis-attenuated to avoid bias (Schielzeth, 2010). Un-standardized regression coefficients (Schielzeth, 2010) were then used to measure the effects of each predictor variable: attachment style, emotional intelligence, and resilience on time perception. A covariance matrix, collinearity diagnostics, and part and partial correlations were performed to determine whether multicollinearity (Licht, 1995) bias introduced large standard errors in the prediction model. Two violations of multiple linear regression occurred so step-wise logistic regression analysis was used instead.

\section{Significance of the Study}

This study has both theoretical significance and clinical application. Its most important contribution was to fill a gap in Zimbardo and Boyd's (1999; 2008) model of time that purports that time perspective is related to and in some cases predicts certain human behaviors. The past 30 years of research has been aimed at establishing this linear approach and ascertaining which behaviors result from which time perspective. The current study expanded their model by identifying other variables that may be responsible for establishing, altering, and sustaining time perspective throughout the life span, thereby explaining questions that the model could not previously account for. One such question in the area of academia is, why does the structure of school help some individuals succeed while it hinders others? More specifically, why does school help discipline some students (e.g., by helping them move from a present-hedonistic to a more future orientation) but not all students? This current study provides additional insight into the phenomenon of flexibility of time perspective that some individuals seem to possess while others do not. Instead, the latter remain fixed in a particular bias. On a broader scale, this study opens the door for other researchers to investigate these and other variables that may be responsible for influencing time perception, which in turn may influence many other human behaviors.

The identification of time perception predictors and their relationship to doctoral distance learners in the coursework versus dissertation phase of learning is of clinical interest because of the high attrition rate that occurs during the latter and the potential to change maladaptive time perspectives into those that better support the ability to complete an online dissertation. Many students (over 6.1 million in the fall of 2010 (Allen \& Seaman, 2011)) have pursued distance learning. The growth rate of online learners from 2009 to 2010 was $10 \%$ compared to a $1 \%$ growth rate in classical, higher education during the same time frame according to the same report. Many of these students chose distance learning doctoral programs over traditional classroom instruction because they could complete their coursework and dissertation during time that was convenient for them instead of attending class in a set place and during a particular time (Kvavik \& Caruso, 2005). Asynchronous distance learning programs afforded students the leeway to continue to participate in various life roles that did require strict adherence to a set schedule, such as work or child care, while engaging in school work during free time (Aragon \& Johnson, 2013). A flexible schedule was one of the top four most common factors influencing distance learning program enrollment as reported by the U.S. Department of Education, Institute of Education Sciences, National Center for Educational Statistics (NCES) (Parsad \& Lewis, 2008). Even though the ability to 
study whenever a student desires sounds convenient, more students fail to complete distance learning programs than traditional classroom education (Cockran, 2009) due to an inability to manage time or to anticipate the amount of discipline necessary to turn in assignments without an imposed deadline (Cockran, 2009; Street, 2010).

There may be additional reasons related to time that make it difficult for students to complete distance learning doctoral programs. Students may be unable to maintain a balance of time in the areas of work, leisure, responsibilities, school work, and sleep, which has been found to be important for maintaining stress reduction, psychological need satisfaction, and role balance necessary for physical and mental health (Christiansen \& Matuska, 2006). Another may be related to how students experience their daily activities. If a majority of their daily time is spent in activities that cause them to feel anxious, depressed, angry, or bad about themselves, they may experience difficulty continuing the program. If the number of hours in a typical day is predominantly filled with activities that result in students feeling positive affects, they may be more motivated to achieve their educational goals. Some students may require more structured time. Coursework tends to be more structured than the research involved in a dissertation. Therefore, some students may perform better during the coursework phase of a doctorate than the dissertation phase or vice versa if they perform more efficiently given unstructured time. Therefore, other factors such as emotional intelligence, resilience, and attachment styles may be influencing time perspective.

\section{Definitions}

Asynchronous distance learning programs. Asynchronous distance learning programs are academic programs in which students complete coursework online on their own time instead of with other classmates at the same time as in synchronous distance learning or classical instruction (Zandieh \& Habibi, 2011). Technologies for asynchronous distance learning include but are not limited to videoconferencing, chat, audiocassette, videocassette, mp3s, telephone, message board forums, voicemail, fax, and print materials.

Attachment style. Attachment style is the characteristic manner in which an individual bonds with others (Levy et al., 2011). Attachment serves as a survival mechanism and is determined by early childhood experiences with the primary caretaker.

Distance learning. Distance learning (also referred to as "distance education", "online learning", "electronic learning", "remote learning", or "e-learning") is an educational method that allows students to learn in a place different from the teacher (Moore \& Kearsely, 2012). Methods of instruction may include computer technologies, videos, correspondence through discussion groups, CD-ROMs (National Center for Education Statistics, U.S. Department of Education, 2007). There are three main types of distance learning: asynchronous, synchronous, and mixed or hybrid.

Emotional intelligence. Emotional intelligence is the ability to identify and regulate emotions and to use emotional awareness of self and others to problem solve and engage in relationships (Côté et al., 2011).

Future-orientation. Future-orientation is a category of time perspective that describes individuals who possess the ability to delay gratification in order to achieve goals and objectives, meet deadlines, and plan for the future (Zimbardo \& Boyd, 2008).

Past-negative. Past-negative is a category of time perspective that describes individuals who tend to dwell on past failures, insults, and regrets and have difficulty moving past missed opportunities (Zimbardo \& Boyd, 2008). 
Past-positive. Past-positive is a category of time perspective that describes individuals who tend to perseverate on positive events that occurred in the past, follow tradition, and previously established family values (Zimbardo \& Boyd, 2008).

Present-fatalistic. Present-fatalistic is a category of time perspective that describes individuals who have difficulty taking an active role in life because they believe in determinism and have an external locus of control (Zimbardo \& Boyd, 2008).

Present-hedonistic. Present-hedonistic is a category of time perspective that describes impulsive individuals, have short-term gratification, and strive to satisfy their current needs. These characteristics lend themselves to pleasure seeking, risk taking, and sensation seeking (Zimbardo \& Boyd, 2008).

Resilience. Resilience is the process by which an individual interacts (copes) with the environment that includes factors that promote well-being and include risk (Zautra, Hall, \& Murray, 2010).

Time perspective. Time perspective is the subjective psychological process by which people make sense of personal experiences by putting them into different temporal categories, or time zones. It is one aspect of time that contrasts with objective and inter-subjective views of time (Zimbardo \& Boyd, 2008).

\section{Summary}

The perception of time (the use of temporal categories to conceptualize experiences) affects human behavior (Zimbardo \& Boyd, 2008). The application of time perception to academia is of interest because distance learning programs are becoming prevalent; however, the attrition rate for all but dissertation distance learners has been reported to be as high as $80 \%$ (Flood, 2002). Students with future time perspective orientations tend to have better academic outcomes than those with past or present according to Zimbardo and Boyd's $(1999 ; 2008)$ psychology of time model. However, this model may be limited because of the exclusion of certain variables that may influence the development and maintenance of time perspective and hence, academic achievement. Their model claims that time perspective continually develops and changes but does not explain which aspects of structure and discipline affect time perspective or its plasticity.

This nonexperimental quantitative survey ascertained whether emotional intelligence, attachment style, and resilience operate differently to predict time perception in a structured (coursework) versus the less structured (dissertation) phase of doctoral distance learning, since each of these variables have been shown to influence behavior and relate to time perspective (Fortunato \& Furey, 2010; Laghi et al., 2009; Monahan et al., 2010; Padawer et al., 2007). The results provide clarification to and expand upon Zimbardo and Boyd's theory of time and address the high attrition rate of online doctoral programs. 


\section{Chapter 2: Literature Review}

The purpose of this nonexperimental quantitative survey study was two-fold. First, it was used to identify what predictive role emotional intelligence, attachment style, and resilience (predictor variables) had on time perception (criterion variable) in doctoral distance learners. Second, the study was used to ascertain how emotional intelligence, attachment style, and resilience (predictor variables) interacted to predict time perception (criterion variable). The results provide clarification to Zimbardo and Boyd's model of time (1999; 2008) by uncovering the predictive role of attachment style, emotional maturity, and resilience on time perception during a structured phase (coursework phase) and the less structured phase (dissertation phase) of doctoral distance learning. The literature review discusses each of these key topics/variables in sequential order: Distance Learning, Time Perception, Emotional Intelligence, Academic Achievement, Attachment Style, and Resilience.

\section{Documentation}

This review of the literature was based on literature searches performed using the following search terms individually and in combination: attachment style, distance learning, emotional intelligence, psychological resilience, and time perspective. The following databases were searched for appropriate publications: Annual Reviews, Education Research Complete, PsycARTICLES, PsycBOOKS, PsycINFO, EdIT Digital Library, ERIC, ETS Test Link, Gale Academic OneFile, JAMA: Journal of the American Medical Association, MEDLINE, Mental Measurements Yearbook with Tests in Print, Pro Quest, PsychiatryOnline, Psychological Reports, Psyc TESTS, ScienceDirect, Taylor \& Francis Online, Teachers College Record, Wilson OmniFile Full Text, and WorldCat. In order to report the strength of correlation results consistently between different articles throughout the literature review, Green and Salkind's (2011) delineation of strength between two variables was used. Green and Salkind stated that Pearson Correlation Coefficients of 0.10, 0.30, and 0.50 are interpreted as small (weak), medium (moderate), and large (strong), respectively, regardless of direction of the relationship. Green and Salkind's delineations were used in this study because they are conventionally used by behavioral scientists.

\section{Distance Learning}

A review of literature on distance learning is necessary in order to understand its prominence in today's educational system and the public domain. Since participants of this study were online distance learners, it is important also to understand the nature and structure of some of the current systems and how they originated. The literature review on distance learning, therefore, includes a history of distance learning and a subsection on current online distance learning.

History of distance learning. Distance learning did not originate with the advent of the Internet. Instead, it has a long-seeded history that dates back hundreds of years. In order to establish its time and place of origin, a definition of distance learning must be given. According to Moore and Kearsley (2012), distance learning is the teaching and acquisition of knowledge that occurs in different locations by learning institutions through the use of technology. Letters, radio, television, phone teleconferences, and computers are technologies that have developed over time and have been instrumental in communicating education across distances (Bowers, Freyman, McLellan, Paxton, \& Spiegel, 2013). Although there is slight controversy, the first instance of distance learning was reported by Bowers et al. (2013) to be an advertisement listed in The Boston Gazette on March 20, 1728, by a teacher living in the city for instructions given by mail to country-folk to acquire the skill 
of short-hand. In the 1800 s, teacher Isaac Pitman taught Bible studies via mail in Great Britain (Power \& Gould-Morven, 2011). In 1858, the University of London took Pitman's idea and began to offer correspondence courses that students could complete at home, as noted by Bowers et al. (2013). Women's educational needs were addressed by the development of the Society to Encourage Study at Home. This program, taught by educated women offered women 20 different courses that could be completed at their own pace at home, according to Bowers et al. (2013). John Vincent developed what would become the first long-term degree offering distance learning four year program in New York in 1878 in accordance with the Chappaqua Literary and Scientific Circle, as stated by Bowers et al. (2013). As brick and mortar institutions gained popularity, they began to offer correspondence courses but most of them focused on a particular skill. It was not until the invention of the radio in the early twentieth century, according to Bowers et al. (2013), that federally issued radio education licenses were issued (the first being 1921) and the development of aural distance education followed. Educational credit for completing a radio course was first offered in 1925 by the University of Iowa, which later offered television education in the form of telecourses, according to Power and Gould-Morven (2011).

A major milestone in the development of distance learning was the advent of the British Open University in 1969 with its new evaluation system, technologically advanced method of instruction, and long-term degree programs that proved that students could receive quality instruction without being present in a classroom (Bowers et al., 2013). Distance learning became global in the 1970s with the invention of teleconferencing. Countries such as Pakistan, Sri Lanka, Japan, South Africa, West Germany, and Canada opened distance learning programs (Moore \& Kearsely, 2012). Other countries, such as Turkey (Anadolu University with over 500,000 distance learners in 2005) and Hong Kong (Open University of Hong Kong with over 100,000 distance learners in 2013), followed suit, according to Bowers et al. (2013).

Another major contributor to the advancement of distance learning was the World Wide Web, which allowed faculty to teach students globally with a click of a button and utilized advanced portal systems to disseminate and store course information throughout a virtual university (Bowers et al., 2013). Until this major invention contributed to education, distance learning was an adjunct to classical learning, but with the use of the Internet, online distance learning began to compete with classical learning and has been growing at a faster rate since 2002 (Allen \& Seaman, 2011).

Current online distance learning. Online distance learning courses are quickly replacing courses taught through classical learning throughout higher educational institutions in the US (Allen \& Seaman, 2011). Sixty-five percent of universities in America reported online distance learning to be an important focus of their strategic plans, according to Allen and Seaman (2011). A widely utilized novel occurrence in online distance learning is the initiation of the Massive Open Online Course (MOOC), which began several years ago to offer free online courses to the public. Many university professors have uploaded videos of their lectures and multiple choice exams creating packaged online courses (Bowers et al., 2013). Massachusetts Institute of Technology and Harvard University offer MOOCs through a program called "edX" (Lewin, 2012). MOOCs are also offered through the Coursera Program established to offer free, high quality, online courses to individuals that would otherwise not be able to receive the education because of financial, geographic, and time restraints (Severance, 2012). Universities that currently offer Coursera Programs include Harvard University, University of Pennsylvania, University of Michigan-Ann Arbor, and Princeton University (Selingo, 2012), but Coursear is also being implemented internationally. The Kahn Academy was developed in 2009 to enhance classical 
learning by offering videotaped lecture tutorials to students online ahead of time so that face-to-face class time instead of professor-led lectures, could be used to stimulate class discussion (Bowers et al., 2013). Currently, there are more than 3,000 videos available for viewing with over six million online distance learners per month in the Kahn Academy, according to Bowers et al. (2013). Over 10,000 students may be enrolled in the same MOOC at the same time, so academic integrity is a problem (Belkin, 2013). Despite this problem, which universities and individuals are working to solve, MOOCs, according to Pappano (2012), have become established forms of higher education.

While individuals and institutes of higher education are working hard to improve the quality of education that MOOCs provide and use the best technology available to present their courses, MOOCs do not culminate in degreed programs. Instead, MOOCs are stand alone courses and can be taking at the discretion of the student in any order. There are, however, many online distance learning degreed programs offered by accredited higher educational institutions. There are over 307,000 online distance learning students enrolled in the University of Phoenix ("University of Phoenix online", 2012). At the time of this study, there were at least 25 different online distance learning programs that offered Ph.D. degrees. Like the MOOCs, online distance degree programs are growing in quality and quantity, but their dropout rate is higher than that for classical learners (Cockran, 2009; Nichols \& Levy, 2009) and may, in part, be due to poor time management skills (Barbour \& Reeves, 2009; Cockran, 2009; Long et al., 2009; Street, 2010), which may be predicated upon the perception of time.

\section{Time Perspective}

The theoretical considerations of time perspective include a summary of the behavioral research on time, Zimbardo and Boyd's perception of time model (1999; 2008), and a balanced time perspective. Behavioral research on time includes objective, subjective, inter-subjective, and interactive or multidimensional viewpoints. Zimbardo and Boyd's perception of time model includes descriptions of its theoretical origin, temporal categories, and possible temporal biases. A theory of balanced time perspective and research using a balanced time perspective model is discussed as are the relationships between age and gender on time perspective. Additional models of future time perspective are included.

Behavioral research on time. Behavioral research on time can be categorized into four different views: objective, subjective, inter-subjective, and interactive. The objective view examines measurable clock time and includes internal clock models such as the scalar expectancy theory, which purports that an internal pacemaker in the brain sends rapid repetitive pulses to an accumulator via a switch that opens and closes in reaction to a stimulus; the more pulses, the greater the duration of the stimulus (Wittmann, 2009). However, different variables such as arousal, memory, and attention can change the pulse rate or affect the switch in such a way as to alter the subjective experience of time. Arousal-mediated temporal overestimation occurred when the level of physiological activation increased resulting in an acceleration of the internal clock caused by an increase of accumulated pulses over the same unit of objective time (Ortega \& Lopez, 2008). For example, arousing faces showing anger and fear resulted in an overestimation of their duration compared to neutral faces (Droit-Volet \& Gill, 2009), and fear (Campbell \& Bryant, 2007) and disturbing sounds (Noulhiane et al., 2007) slowed the pace of time causing an overestimation of temporal intervals. Attention-induced underestimation occurred when subjects were distracted from the experience of time, which may have resulted in a temporary switch opening or failure to close causing pulses to be lost and the duration to be judged as shorter (Buhusi \& Meck, 2009). As far as memory is concerned, the amount of energy necessary to process an event was proportional to 
its perceived duration; repeated exposure led to predictability, which shortened subjective duration and vice versa (Eagleman \& Pariyadath, 2009). Consequently, the greater the difference between a stimulus and its background, the greater the distance effect (Matthews, Stewart, \& Wearden, 2011).

According to Lee and Ji (2013), time perspective is subjective and, therefore, different for every individual. Their study examined whether or not there was a correlation between the way people perceived past and future time and if that prompted ego-moving perspective of time, where people saw themselves as moving from past to future while time stood still, or time-moving perspective of time, where people saw themselves as standing still while time moved them from future to past. The authors concluded that a positive past and a negative future time perspective prompted a time-moving perspective of time while a negative past and positive future perspective prompted an ego-moving perspective of time.

The inter-subjective view explores the effect that other people have on an individual's experience of time. For example, age may play a role in creating a temporal bias, such as in a study with college students who judged their exposure to elderly faces as shorter than their judgment of youthful faces even though the duration was the same for each exposure (e.g., Chambon et al., 2008). The findings suggested that college students held the perception that older people move slower, thus their experience was influenced by their subjective perceptions.

The interactive view proposed by Ballard and Sibold (2006) integrated the objective, subjective, and inter-subjective perspectives of time to explore how environmental occurrences (e.g., work culture, norms, personality, perception of time, social interactions) effect time perspective. For example, their findings indicated that greater job satisfaction was characterized by individuals who were more punctual and categorized their work as future-oriented; whereas, individuals who were flexible experienced work delays, and viewed their time as future-oriented reported greater communication loads. In contrast, individuals who were linear in job related tasks and were strongly future-oriented experienced the most satisfaction with communication among departments. Given the evidence above, it appeared that time is not a mental construct, but involves active, ongoing derivations of estimates from multiple internal and external sources of information (Wittmann, 2009; Wittmann \& Wassenhove, 2009).

Zimbardo and Boyd's perception of time. Zimbardo and Boyd (2008) believed that time perception was multidimensional. Their psychology of time theory incorporated five theories. They were: social psychologists' belief that time was a social construct (Moscovici, 1984), Kant's theory (1781/1965) that time affected the individual's view of the world, Lewin's theory (1951) that the perception of time directly influenced behavior, cognition (Nuttin, 1964), and self-efficacy (Bandura, 1977).

According to Zimbardo and Boyd (1999), time perception is the learned process by which individuals unconsciously sort personal experiences into temporal categories or time zones in order to create meaningful, coherent, and organized events. Zimbardo and Boyd developed the ZTPI (1999; see Appendix A) to measure individuals' orientations to the past, present, and future. These time perspectives were found to include past-negative, past-positive, present-fatalistic, present-hedonistic and future categories (Zimbardo \& Boyd, 1999) and have been described by quantitative and qualitative (case studies) research utilizing undergraduate students by Zimbardo and Boyd $(1999 ; 2008)$ as summarized below.

Individuals with a predominantly past time orientation tended to see the world through their past, which can be viewed as negative (past-negative) or positive (past-positive). Students with a past-negative orientation were characterized as negative, pessimistic, and having an unfavorable attitude towards their past. Individuals 
with a past-negative orientation tended to have poor object relations. They had significantly fewer close friends than their peers. They reported having sex less frequently; all of the $60 \%$ of the participants in the study who reported not having sex scored high in past-negative. And, they tended to favor experiences over people. Past-negative had a strong positive correlation with depression, unhappiness, anxiety, aggression, and low self-esteem. Overall, people with a bias in past-negative time perspective had difficulty enjoying life in the moment as compared to participants with other time perspective biases.

Individuals with a past-positive orientation focused on positive memories of experiences from the past. They tended to be introverted and shy yet reported good object relations. When compared to individuals with other time perspectives, past-positive individuals tended to be more spiritual, involved in relationships, and married. They approached life in a safe manner, not taking many risks. They had less sex and fewer sexual partners than people in most of the other time perspective biases. They also drank less alcohol. Past-positive orientation showed a moderate positive correlation with self-esteem. Students with a past-positive orientation had a healthy, happy outlook on life as contrasted by those with high scores on present-fatalistic and past-negative orientations.

Those with predominantly present time orientations were focused in the present (Zimbardo, 2007). Present-fatalists tended to view the present in a negative way, felt dissatisfied with life, and believed that nothing could be done to improve their current situations or the future (Zimbardo \& Boyd, 2008) even though they had a substantial degree of self-efficacy and even though their current situation was often viewed by others as fairly good. Students with a present-fatalistic time perspective believed that they needed to resign to fate because the future is predestined and out of their control. This factor showed a strong positive correlation with anxiety, depression, consideration for future consequences, and aggression. Students with present-fatalistic time perspectives were much more likely to have clocks on their desks and had much lower grade point averages. They wished to live shorter lives and did not want more time to spend with friends. They tended to be promiscuous and not practice safe sex. Present-fatalistic time perspective has been considered the as the absence of a defined time perspective because it lacks the excitement of the hedonistic-present, goal focus of the future, and bitterness or nostalgia of the present orientations.

Present-hedonists lived for the moment with little regard for the future, so they tended to seek pleasure, excitement, and enjoyment while avoiding pain. They tended to have unclear goals for the future, be atheist or agnostic, consumed more alcohol, have divorced parents, not wear wristwatches, and communicated with their families more frequently. They had a lot of energy and often engaged in multiple sports and activities, more so than individuals in other time perspective categories. Present-hedonistic time perspective showed strong positive correlations with novelty seeking, lack of ego control, sensation seeking, and a lack of preference for consistency.

Future time oriented individuals thought of the world in terms of their future and often delay gratification in the service of long-term goals (de Bilde et al., 2011; Zimbardo \& Boyd, 2008). These individuals tended to get and achieve long-term goals, be ambitious, and be very organized (Zimbardo \& Boyd, 1999). While they admitted feeling the pressures of time, they were willing to forgo present satisfaction for long-term goal attainment in order to advance their careers, according to Zimbardo and Boyd (1999). Zimbardo and Boyd's (1999) research also revealed the following information about students with future time perspectives. When compared to their peers, future oriented students were more efficient, better at planning, made to-do lists, utilized a daily calendar, wore wristwatches, balanced their checkbooks, had more structure and order in their 
daily lives, and had the most defined goals. Because they tended to have higher standards and experienced pressure from time limits, future oriented students tended to experience more stress than students with other time orientations. They reported having little free time because they filled their time with goal directed activities. Future oriented students' stressful lifestyle paid off in that they had the highest grade point averages and fewer incompletes courses than their classmates. Even though future oriented students felt that it would be healthy to make friends, their hectic overachieving behaviors precluded most of them from making and keeping friends. They also desired to have fewer children, live longer, and eat healthier. Future time perspective correlated with consideration of future consequences (strong, positive), conscientiousness (strong, positive), preference for consistency (moderate, positive), number of hours spent studying per week (weak, positive), sensation seeking (moderate, negative), novelty seeking (moderate, negative), depression (weak, negative), and anxiety (weak, negative). There was no significant correlation found between future time perspective and aggression.

Another assessment tool that identifies time perspective is the S-ZTPI (Carelli, Wiberg, \& Wiberg, 2011), which took into account the possibility of not only a future, but future-positive and future-negative constructs. Carelli et al. (2011) identified that the ZTPI's future time perspective was focused on and limited to thinking positively about the future. As past and present time orientations are divided into positive and negative subscales, Carelli et al. (2011) reasoned that the future construct could have a negative aspect, as well. Carelli et al. (2011) considered hope and anticipation of success as thinking positively about the future and fear, anxiety, concerns, worrying about the future, or expecting less than ideal results, as thinking negatively about the future. Carelli et al. (2011) administered the S-ZTPI to 419 people between the ages of 18 and 80 in a moderate-sized town in Sweden. Carelli et al. (2011) used a procedure known as backtranslation to verify accuracy of the translation of the ZTPI into Swedish. Backtranslation involves professionally translating the original material into another language and then back to the original language, by a second professional translator. Two future items (numbers 9 and 18) from the original ZTPI were combined with eight original items to comprise the new future-negative scale in the S-ZTPI; the other 11 future items from the original ZTPI made up the future-positive scale of the S-ZTPI. To ensure validity of the S-ZTPI, participants were asked to fill out additional surveys, which determined their self-reported impulsivity (via the BIS-11 scale) and decision-making styles (via the GDMS instrument). The S-ZTPI (Carelli et al., 2011) was shown to have a strong correlation $(r=0.96)$ between the future scale of the ZTPI and the future-positive scale of the S-ZTPI, but a weak correlation $(r=0.12)$ between the future scale of the ZTPI and the future-negative scale of the S-ZTPI. Expected correlations were found to exist in this study between impulsiveness and decision-making styles and time perception; individuals who were present-oriented tended to be more impulsive and individuals who were negatively-oriented tended to be avoidant and dependent decision makers. Future-negative time perspective seemed to constitute another aspect of time perspective, as measured by the S-ZTPI, which was shown in this study to be a standardized, reliable, and valid measure of time perspective.

Whether measured by the ZTPI or the S-ZTPI, the predominant type of time perspective an individual acquired in early development lasted into adulthood unless certain learning situations in life or conscious efforts altered the perspective as demonstrated by early delayed gratification experiments with children (Zimbardo \& Boyd, 1999) and longitudinal follow-up reports (Zimbardo \& Boyd, 2008). This is because time perspectives are used to store, encode, and recall experiences; imagine outcomes; set goals; shape expectations; make decisions; feel; and be. In this way, individuals' lives are shaped are by their perception of time. 
Optimally, the present consists of empirically centered, concrete representations built from psychological, abstract constructions of the past and future expectations. Yet, individuals may over-develop one particular time perspective such as the past, present, or future (temporal bias) and have difficulty switching between them when necessary. Education, social class, culture, religion, family modeling (e.g., attachment style, resilience), and other learned factors may determine this overreliance. Historical and cultural experiences were found to have shaped future time perspective in Polish students (Stolarski et al., 2011). If habitual, a dispositional style can arise that is predictive of many behaviors as noted above. This is in contrast to a balanced time perspective that can change according to circumstance.

\section{Balanced Time Perspective}

A balanced time perspective is one without biases towards any particular time perspective (Stolarski et al., 2011). It is one in which attitudes towards the present, future, and past are interconnected so that flexibility among all three can be achieved under different circumstances that may require different perspectives (Boniwell, Osin, Linley, \& Ivanchenko, 2010). The possession of a balanced time perspective is considered ideal because it implies choice and control over one's perception of time, allowing individuals to better adapt to changing situations (Zhang, Howell, \& Stolarski, 2013). The art of balancing time perspective involves the mental ability to consciously switch time perspectives based on personal resources, the context of a situation, and features of a task (Zimbardo \& Boyd, 1999). A time perspective bias infers that only one particular time perspective is available to an individual regardless of the setting and task (Zimbardo \& Boyd, 1999) because the other time perspectives are underdeveloped and, therefore, not accessible for use (Zimbardo \& Boyd, 2008). A time perspective bias is not adaptive in different circumstances. A balanced time perspective, however, allows an individual to feel grounded in a sense of identity, tradition, and culture from contributions of a past positive; a future orientation allows for a sense of excitement looking forward to achieving future goals; and daily nurturing through a sense of playfulness and sensuality can be achieved through contributions of the hedonistic-present, as explained by Zimbardo and Boyd (1999). Possessing different time perspectives and a fluidity between them is not enough, according to Zimbardo and Boyd (1999). Fully actualized individuals working with their full potential must also possess or develop harmony among time perspectives so that they all work in consort (Zimbardo \& Boyd, 1999).

According to the balanced time perspective model proposed by Shirai, Nakamura, and Katsuma (2012), an individual should be able to live a full life in the present while also striving for future goals. Therefore, a student who has a balanced time perspective would have the ability to delay gratification in order to study for an upcoming exam yet be able to enjoy a party afterwards. The same student may use negative past academic experiences to improve future academic circumstances while still being able to reminisce about past-positive and pleasurable memories to feel better in the present. However, Zimbardo and Boyd's research has shown that students' academic success was related to a future time perspective bias and not a balanced time perspective (1999); instead, a balanced time perspective was related to success in adult life in general but not academically (Zimbardo \& Boyd, 2008).

The Balanced Time Perspective Scale (Webster, 2011) was created and found to be a valid and reliable measure that determined the balance between past-positive and future time perspectives within an individual. This scale measures the extent to which a time perspective profile deviates from balance through the deviation of a balanced time perspective coefficient (DBTP). The results of Webster's study using the Balanced Time 
Perspective Scale found that people who frequently engaged in positive thoughts about both the past and the future were the happiest, felt best about life, and had high self-esteem. A balanced time perspective was found to be the most health promoting, yet the ZTPI, which measures time perspective, is void of a present scale that measures mindfulness, which has been related to happiness and life satisfaction (Vowinckel, 2012). Vowinckel (2012) found that when calculating balanced time perspective using the DBTP and replacing the ZTPI future scale with Webster's (2011) future scale, a new set of time perspective subscales emerged, which resulted in higher correlations of life satisfaction and mindfulness with balanced time perspective.

A study by Gao (2011) was conducted to determine if there was a correlation between having a balanced time perspective and life satisfaction in the young adult Taiwanese population. It was found that those with strong past-negative and present-fatalistic perspectives had a strong negative association with life satisfaction and those with strong past-positive, future, and present-hedonistic qualities had a strong positive relationship with life satisfaction. Overall, it was concluded that those with a balanced time perspective exhibited higher life satisfaction than those without a balanced time perspective. Additionally, a study conducted by Cretu (2012) was able to confirm a strong positive relationship between future and past-positive time perspectives with a happier lifestyle and higher self-esteem. A balanced time perspective predicted general well-being (Zhang et al., 2013). The construct of a balanced time perspective is a complex one that requires further examination.

\section{Future Time Perspective Models}

Literature reviewed thus far has shown that the construct of Zimbardo and Boyd's (1999) future time perspective has been associated with, and in certain studies predicted, multiple measure of academic success and general life accomplishments. The importance of this construct has resulted in its further examination by multiple researchers. As a result, there have been several models of the construct of future time perspective put forth by different theorists. The first was a result of a limitation of the ZTPI. The ZTPI has two different subscales for its past time perspective (past-negative, which measures negative aspects of past experiences and past-positive, which measures positive aspects of past experiences). It also has two subscales for its present time orientation (present-hedonistic, which measures pleasurable aspects of the present and present-fatalistic, which measured negative attitudes towards the present). Yet, the future construct has only one subscale, which includes items that relate to a mostly positive outlook on the future. Although its reliability and validity has been firmly established over the past 30 years, Carelli et al. (2011) believed that its future dimension should be better defined and expanded. To this end, Carelli et al. (2011) developed the S-ZTPI, which included future-negative and future-positive dimensions because they believed that the future could be viewed as negative as well as positive. The future-negative dimension included items that addressed worrying about the future and anticipating negative events. Even Zimbardo and Boyd (1999) who suggested that additional factor analysis with different non college populations should be performed on the future dimension. The S-ZTPI was found to be a reliable and valid instrument for measuring time perspective (Carelli et al., 2011). Specifically, confirmatory factor analysis showed support for the ZPTI's five-factor solution and Carelli et al.'s (2011) six-factor solution that included the independent factor of future-negative.

In addition to the six-factor model of time perspective proposed by Carelli et al. (2011) that included two future dimensions, a three-component future orientation model was proposed by Seginer (2009). The three components were motivation, cognition, and behavior and their interactions with respect to future attitudes were examined and later applied to the impact of parenting on the development of the future time perspective 
(Seginer \& Shoyer, 2012). Lens, Paixao, Herrera, and Grobler's (2012) model of future time perspective was similar to Seginer's in that it focused on a cognitive-motivational construct of future time perspective with relationship to behavior. Lens et al. (2012) believed that future time perspective is related to and developed through goal setting. Goals are characterized by their content, specifically, what the individual is trying to achieve and by their depth, which is measured by the amount of time each goal extends into the future (e.g., short versus long-term goals). Differences in the depth and content of the goals had an effect on motivation according to Lens et al. (2012). Although there have been different models for the future time perspective construct, literature has included most of them in a theory called the future time perspective theory.

Research has supported the future time perspective theory. Relationships between delayed gratification and development of students' attitudes towards career and school, and importance of learning were noted (Peetsma et al., 2012). Graduate students who had a close connection between their present activities and imagined futures (connectedness) tended to value (instrumentality) their school work and developed complex knowledge structures necessary for life-long learning (Hilpert, Husman, Stump, Kim, Chung, \& Duggan, 2012).

\section{Age and Time Perspective}

Horstmanshof and Zimitat (2007) found that future-orientation in older students positively correlated with higher levels of academic performance in terms of dedication to studies, deeper approaches to learning, and applying course material. Older students studied for larger amounts of time, applied themselves intellectually, were diligent and participated more in the learning community. This finding was attributed to the possibility that older students managed to balance multiple responsibilities better than their younger peers. However, age and future time perspective showed a negative correlation in young adolescents, ages 11 and 14 in a study completed in Northern Italy by Ferrari, Nota, and Soresi (2010). Results were explained by the belief that as a young adolescent matures into an older adolescent, exploring self and the world becomes the focus and therefore the focus on the future decreases.

\section{Gender and Time Perspective}

Gender was significantly associated with future-negative time perspective (Peetsma et al., 2012). Females had significantly less negative thoughts about the future than males. This might be the reason that more females complete school and achieve educational success, according to Peetsma et al. (2012). Ferrari et al. (2010) found the same results with young Northern Italian adolescents; however, as the female adolescents matured into older adolescents, their levels of future time perspective decreased as a result of social pressure.

Females tended to complete academic assignments more promptly than males, and gender compounded with time perspective had an even greater correlation with assignment completion times (Harber et al., 2003). Specifically, future-oriented females completed assignments more quickly than both future- and present-oriented males. Even present-oriented females occasionally completed assignments before future- or present-oriented males in this study. However, differences in gender and time perspective did not significantly correlate with tardiness. Although females tended to be less tardy with assignment completion, they were not more consistent than males when completing longitudinal work, over an extended period of time, as per Harber et al. (2003). The trend of females being more prompt than males, but not as consistent with long-term assignments, was not due to emotional variables, which were ruled out as possible factors influencing student tardiness in academic performance in this study. Emotions were ruled out as contaminating factors because they influence how time is perceived, so emotional intelligence may be a factor that affects time orientation as well. 


\section{Emotional Intelligence}

Emotional intelligence, the ability to identify and regulate emotions and to use emotional awareness of self and others to problem-solve and engage in relationships (Côté et al., 2011), should be related to time perspective. Emotions experienced during a current event should have an effect on the memory of that event. Memories of past experiences effect emotions experienced in the present and the present state of mind influences a projected future.

There is support for emotional intelligence playing a role in time perception. In particular, Zimbardo and Boyd's (1999) study found significant moderate to strong correlations between time perspectives and a variety of emotional experience: (1) past-negative time perspective had a moderate positive association with aggression and depression, a moderate negative association with emotional stability, impulse control, and level of happiness, and a strong positive association with trait anxiety; (2) past-positive time perspective had a positive mild association with happiness; (3) present-hedonistic time perspective had a strong positive association with ego control; and (4) future time perspective had a positive moderate association with aspects of self-control and delayed gratification (e.g., consideration of future consequences, preference for consistency) and a mild negative association with novelty seeking (Zimbardo \& Boyd, 1999).

Worrell and Mello (2007) found somewhat similar correlations between time perspectives and emotional intelligence in that: (1) past-negative time perspective had a moderate positive association with perceived stress and a moderate negative association with hope, self-esteem, and optimism; (2) past-positive time perspective had a mild negative association with perceived stress and a moderate positive association with hope, optimism, and self-esteem; (3) present-positive time perspective had a strong negative association with perceived stress, a strong positive association with hope, a moderate positive association with optimism and self-esteem, and a mild positive association with the belief that the future will work out; (4) present-negative time perspective had a very strong positive association with perceived stress, a strong negative association with optimism, and a moderate negative association with hope and self-esteem; (5) future-positive time perspective had a moderate negative association with perceived stress and a moderate positive association with hope, optimism, the belief that the future will work out, and self-esteem; and (6) future-negative time perspective had a strong negative association with self-esteem, a moderate positive association with perceived stress, and a moderate negative association with hope, optimism, and the belief that the future will work out.

A study conducted by Klapproth (2011) examined the different roles that emotion can play in determining time perspective. Klapproth found that the mood of the participants affected how they viewed the future. It was concluded that those in a positive mood placed more value on the future than those in a negative mood. Hicks, Davis, Trent, and King (2011) conducted a study to determine if positive affect would relate more strongly to meaning of life when time is perceived as limited. Four separate studies were conducted. The results of the first study showed that positive affect was significantly correlated to meaning in life for older participants than younger participants. The results of the next two studies showed that positive affect was strongly correlated to meaning in life for those who believed to have limited time left when compared to those who viewed their time as unlimited. The results of the last study showed that positive affect was strongly correlated to meaning in life for those who believed the opportunities to pursue their goals were limited when compared to those who viewed their opportunities as unlimited. Overall, Hicks et al. (2011) concluded that positive affect is strongly related to meaning in life, especially as future time becomes more limited. 
Time perspective was thought to play a role in structuring emotional experiences and influencing personality, according to Stolarski et al. (2011). Time perspective influenced anticipation and recall of mood: past-negative orientation was related to anticipating negative moods while a past-positive orientation was associated with the anticipation of energy and recall of energetic states (Stolarski et al., 2011). Future time orientation predicted energetic arousal, but only in the absence of present-hedonism while balanced time perspective was associated with positive affective states in the same study. Present-hedonistic and past-negative time orientations were the strongest predictors of current emotional states, according to Stolarski et al. (2011).

Direct correlations between emotional intelligence and the five factors of time perspective were documented (Stolarski et al., 2011). Specifically, future, present-hedonistic, and past-positive orientations showed a weak positive correlation to emotional intelligence. A past-negative orientation showed a strong negative correlation to emotional intelligence. A present-fatalistic orientation showed a moderate negative correlation to emotional intelligence. Finally, results demonstrated a strong positive correlation between a balanced time perspective and emotional intelligence. Interestingly, the combination of time perspectives, time perspective balance, and emotional intelligence did not predict the ability to delay gratification in this study.

Kairys (2010) examined the correlation between time perspective and personality traits in two adult age groups. Five-hundred and forty-five participants were divided into two age groups: under 35 and over 35 . Extroversion and future time perspective correlated only in the younger group. Extroversion and past-positive time perspectives correlated only in the younger group. Openness to experience correlated with hedonistic time perspective in both age groups; however, the correlation was stronger in the younger group than in the senior group. Conscientiousness and future time perspective correlated in both age groups; however, the correlation was much stronger in the younger respondent group than in the senior group. Conscientiousness and past-negative time perspective correlated only in the younger respondent group. The outcome of this research supports that time perspective is a stable phenomenon and is constructed similarly to personality traits.

In contrast to Kairys' study (2010), Zhang and Howell (2011) reported that extroversion was moderately and positively correlated with both future and past-present time perspectives in adults. Life satisfaction showed a strong positive correlation with individuals who were extroverted and scored high on past-positive and present-hedonistic time perspective dimensions of the ZTPI while individuals less satisfied with life tended to score high on neuroticism and past-negative time perspective, according to Zhang and Howell (2011). Their results also showed that time perspective was a partial mediator in the relationship between life satisfaction and personality. Time perspective also accounted for $13.7 \%$ of the variance while personality traits accounted for $4.4 \%$ in a linear regression model that attempted to explain life satisfaction.

Barber, Munz, Bagsby, and Grawitch (2009) examined how individuals' self-control influenced the correlation of academic achievement and time perspective using the self-regulatory framework. Results showed that future time perspective was positively associated with college grade point average and self-control, whereas the relationship between present-hedonistic time perspective and self control was not statistically significant. Results also supported Zimbardo and Boyd's finding that future-oriented students were more likely to have higher grade point averages than present-oriented students who were not as successful academically.

Coudin and Lima (2011) analyzed data (43,000 responses from individuals in 23 countries) in order to explore how subjective well-being impacted motivationally consistent goals. In order to assess the level of development of each country, the researchers also used the Human Development Index in the year the data was 
collected (2006). Questions relating to future time perspective offered graded response choices on either an 11or 5-point scale. Findings corroborated earlier research findings that individuals with a future time perspective showed the greatest consistency with social goals. The research also indicated that individuals who maintained a future time perspective that was open-ended had a greater sense of well-being than those with a limited future time perspective who were more likely to be depressed. Results demonstrated that emotional state can greatly impact success in any future-oriented action.

Additional findings (e.g., Xie et al., 2010) have indicated that time perspective partially mediated the relationship between belief in a just world and hope, depression, and anxiety. Specifically, the Xie et al.'s (2010) study found that a high score in belief in a just world predicted a strong future time orientation, which predicted high scores in hope and low scores in depression and anxiety scales in Chinese undergraduate earthquake victims. For all three variables, hope, depression, and anxiety, future time perspective predicted more of an effect than belief in a just world alone. A moderate positive correlation was noted between future orientation and hope, a moderate negative correlation was noted between future orientation and depression, and a mild negative correlation existed between future orientation and anxiety.

Emotional stability has predicted different time perception types. Initial validation studies of the ZTPI (Zimbardo \& Boyd, 1999) examined the relationship between the Big Five personality traits (openness to experience, conscientiousness, extraversion, agreeableness, and emotional stability) and the five time perspectives of the ZTPI. Many correlations were reported, yet the predictive nature of these personality traits on time perspective biases was not examined. In order to expand on Zimbardo and Boyd's validation study, Dunkel and Weber (2010) examined personality in the prediction of time perspective. Results indicated that personality was responsible for $55 \%$ of the variance in time perspective. With regard to emotional intelligence represented by "emotional stability" on the Big Five personality traits, Dunkel and Weber (2010) reported that emotional stability, which they termed "neuroticism", was involved in predicting three of the five time perspective biases: future, past-fatalistic, and past negative. Specifically, emotional stability moderately predicted future orientation in the positive direction along with conscientiousness (strong, positive), and identity (weak, positive). Emotional stability moderately predicted past-negative time perspective in the positive direction along with extraversion, which had a weak negative prediction. Emotional stability weakly predicted past-fatalistic time perspective in the positive direction in conjunction with conscientiousness (moderate, negative) and extraversion (weak, positive).

Emotional intelligence was reported to be important to the development of a balanced time perspective (Stolarski et al., 2011). A balanced time perspective predicted general well-being (Zhang et al., 2013). Specifically, a balanced time perspective was related to an increase in happiness, gratitude, vitality, self-determination, positive affect, psychological need satisfaction, and life satisfaction as well as a decrease in negative emotions as reported by Zhang et al. (2013). This balancing of time perspective is considered unconscious (Zimbardo \& Boyd, 2008), yet others believe it can be created (Boniwell et al., 2010).

One of the ways that a balanced time perspective could be created is through the regulation of emotions, according to Stolarski et al. (2011) and is explained below. Emotional regulation, which is necessary to delay gratification, is a component of emotional intelligence. In order to obtain a realistic balance between future consequences and current needs, the regulation of emotions is necessary in order to stave off impulses of immediate gratification. In order to judge which path to follow, present hedonism or delayed gratification in order to achieve a long-term goal, the ability to step out of a current emotional dilemma is required. In order to 
develop such an observing ego, emotional regulation is required in order to adequately remove one's self from the current situation in order to reflect on the pros and cons of both sides of the situation. Possessing a high level of emotional intelligence, then, offers more of an ability to consciously control the switching between different time perspectives. Further flexibility in time perspectives may be acquired through the ability to direct emotions towards focusing attention and recalling necessary memories followed by prioritizing in order to switch time perspectives. Each time perspective has different emotional attitudes (e.g., future and past-hedonistic time perspectives have been associated with conscientiousness, impulse control, and concern for future consequences (Zimbardo \& Boyd, 1999)). Therefore, emotions are associated with decision making processes and their resultant behavioral outcomes (e.g., delaying gratification in order to study). The possession of healthy coping skills (also a component of emotional intelligence) can influence attitudes about the present and future and vice versa. This theoretical discussion was supported by the finding that the ability to delay gratification was predicted by the deviation from a balanced time perspective (Stolarski et al., 2011). Furthermore, emotional regulation, emotional application, and coping strategies were factors found necessary to the development of a balanced time perspective as per Stolarski et al. (2011). Emotional intelligence findings also indicate an association with academic achievement.

\section{Academic Achievement}

The predictors of academic achievement vary across a wide spectrum of constructs. Emotional intelligence will be discussed as a predictor of academic achievement and the relationship between time perspective and academic achievement will be discussed. Attachment style and resilience will be discussed separately in their respective sections.

Emotional intelligence. Some students lack emotional intelligence that would enable them to use their emotions in a way that promotes problem-solving and time management in order to stay in college (Boyd, Gast, Hunt, Mitchell, \& Wilson, 2010). The strongest self-reported barrier to academic success among college students who withdrew from their program before completion was experiencing stress, anxiety, and depression, which was reported by $58 \%$ of the responders, and $27 \%$ of them also reported that these emotions affected their ability to manage time, according to Boyd et al. (2010). Yet, not all aspects of emotional intelligence affect attrition. Only one out of five sub-factors of emotional intelligence, "being aware of emotions", significantly predicted academic outcome in high school students, but "managing emotions", "empathy", "handling relationships", and "motivating oneself" were not significant (Yazici, Seyis, \& Altun, 2011).

Academic achievement and time perspective. Even though students reported valuing online learning (Thompson, Ford, \& Webster, 2011), they had a critical need for self-directed learning. Future time perspective was found to be a helpful tool in promoting educational success (Zimbardo \& Boyd, 1999; 2008). John N. Boyd and Zimbardo (2005) found that people who were the most academically successful tended to have a future orientation, allowing them to see ahead, delay gratification, set goals, and manage their time, whereas present oriented individuals had the highest college attrition rate. Individuals who scored high in future orientation were more punctual for class, apt to complete homework before due dates, inclined to begin assignments on time, and more skilled in time management than their classmates (Harber et al., 2003). The Harber et al.'s (2003) study reported significant gender differences; future oriented females began their assignments before their male counterparts. Future oriented college students also tended to utilize successful learning strategies such as reproductive and deep-level cognitive processing more often than students with other 
time orientations utilized these strategies (Horstmanshof \& Zimitat, 2007). In addition, there was a significant mild positive correlation between grade point average and future orientation among college students (Zimbardo \& Boyd, 1999). Conversely, there was a moderate negative correlation between past-negative and students' report of satisfaction with their educational institutions (Horstmanshof \& Zimitat, 2007). Students oriented to the present were late signing up for, initiating, and completing assignments so tended to receive more incompletes and poorer grades; they were assigned to special education classes more often, and dropped out of school more frequently than their counterparts (Harber et al., 2003). In the Harber et al.'s (2003) study, the poorest grades were more characteristic of fatalists than hedonists.

Mello, Worell, and Andretta (2009) observed a potential mutual nature between time perspective and academic outcomes in an adolescent population. Mello et al. (2009) reported that frequency in thinking about the past among adolescents predicted academic achievement, and that higher frequency of thinking predicted better achievement. Conversely, Mello et al. (2009) found that frequency of thinking about either the present or the future did not correlate with academic achievement. However, Mello et al. (2009) reported that exploring time perspective in the adolescent population was somewhat impaired by the absence of a measurement tool designed for the adolescent population.

Peetsma and van der Veen (2011) observed a strong positive relationship between future time perspective and leisure activities. Contrary to prior literature, there was a weak negative relationship between time perspective and school and career, investment in learning, academic achievement, and social relations when Peetsma and van der Veen (2011) examined first and second year students in a school for special educational in the Netherlands. Low levels of investment in learning and academic achievement with a high level of investment in leisure pursuits could be due to the fact that these students were young adolescents in a special education school on a slower track of education than students in mainstream education. Their levels of motivation, therefore, may be lower, which would lead to lower levels of investment in learning and academic achievement. It seems that leisure was an important life domain for children this age in the Netherlands.

Hannah (2010) reviewed past research literature on factors influencing students' decisions to drop-out or persist in online courses vs. traditional classrooms. External factors (course factors and support), person factors (self-efficacy and autonomy), and academic factors (time and study management) were all linked to persistence in online courses. Instructional design choices appeared to influence students' level of involvement and engagement in a course. Students' internal factors were important for attrition within traditional classroom settings. Self-efficacy was the primary motivational factor for dropping out or persisting in an online course. Results supported Bandura's model, which states that individuals' behavior both influences and is influenced by both personal factors and environment.

Research in the area of time perception and academic outcomes has shown that time perception can be altered through intervention. Horstmanshof and Zimitat (2007) suggested that college curriculum could be enhanced through the addition of various strategies to develop a future time perspective to prevent attrition. Ferrari et al. (2012) developed and tested an intervention strategy to promote future time perspective in adolescent Italian high school students who were selected because of their inability to project their thoughts into the future. The group intervention consisted of 10 two-hour sessions with a psychologist during the school year. The sessions included skill building in the areas of self-awareness, work, goal setting, writing, and building social supports and were found to be effective in switching students' past or present time orientation to a more future bias. 
Admissions criteria are important considerations when determining which students are most likely to do well while in a particular program. Evidence was needed supporting the selection basis to determine what the best indicators of future scholastic achievement in the form of grade point average were while enrolled in a health sciences program. Lysaght, Donnelly, and Villeneuve (2009) evaluated how preadmission criteria correlated with retention in the program, and previous grade point average was found to be a good indicator of student success in the program while previous coursework did not positively correlate with better scores. Results indicated that predictive factors in academic outcomes are not necessarily scholastic based. Therefore, certain personal characteristics and ethical standards can be used as basis for admission boards because they showed positive correlation with student scholastic success. This move away from the efficacy of classic admission criteria showed that there is a valid need for non-traditional acceptance criteria. Redefining admissions criteria promoted academic diversity and more of a chance among the non-academic students to be admitted to colleges in this study.

One such non-traditional variable that may determine success in higher level academia is the extent to which students can manage their time. Beaudoin (2013) found that time management was the second most important factor in academic achievement next to self-motivation. Time management mediated the relationship between time spent studying and academic success (Núñez, Suárez, Cerezo, González-Pienda, Rosário, Mourão, \& Valle, 2013). Additional studies reported that teaching time management skills increased academic (Azar, 2013; Cemaloglu \& Filiz, 2010; Van der Meer, Jansen, \& Torenbeek, 2010; Zampetakis, Bouranta, \& Moustakis, 2010) and professional (Häfner \& Stock, 2010) outcomes. However, Swart, Lombard, and de Jager (2010) reported no significant correlation between academic achievement and time management skills in African engineering students. Their results are in contrast to other studies above whose authors found a relationship between time management and academic outcomes perhaps because of their small sample size $(N=$ 105), the inability to generalize their findings to other populations, and their limited number of time management indicators (10 questions on a self-report survey). Their time management indicators were measures of behaviors (keeping a check list of tasks to do, keeping a study schedule) that typically promote productivity but did not measure productivity itself (number of educational tasks completed per day, number of hours spent studying per day). Although time management is different from the perception of time, the ability to manage time includes factors that have been associated with Zimbardo and Boyd's (1999) time perspectives of students, such as goal setting, delaying gratification, and meeting deadlines.

However, the relationship between time perception and academic achievement may not be so linear. The inclusion of self-determination theory with Zimbardo and Boyd's (2008; 1999) psychology of time theory led to the finding that future time oriented learners regulated their school related activities through internal motives (Ryan \& Deci, 2011), such as feelings of shame and guilt (introjected regulation), interest (intrinsic motivation), and personal conviction (identified regulation). Both present oriented factors were associated with more negative learning and motivational factors (de Bilde et al., 2011). Self-control has been identified as a moderator between time perspective and academic achievement (Barber et al., 2009). Time perception may also have a relationship to attachment style.

\section{Attachment Style}

Bowlby (1979) developed an attachment theory based on an early biological bond formed between caregiver and infant as a protective mechanism from dangerous situations. According to Bowlby (1979), the 
type of bond developed is shaped by the infant's experiences with the primary caregiver. These experiences become internalized and influence the infant's self-image and its image of others (Thompson, 2008). This key topic discusses the link of attachment style with academic achievement, personality factors, and time perspective.

Types of attachment style. Four categories of attachment styles have been described by the literature: secure, avoidant, ambivalent or resistant, and disorganized (Berlin et al., 2008; Bodie et al., 2011). Infants with a secure attachment style tend to believe that their needs will be met and are generally happy, secure, and explorative at baseline. Children with avoidant attachment styles are emotionally distant, rarely explore their environment, and unconsciously believe their needs will not be met. Those with ambivalent or resistant attachment styles tend to be angry, insecure, and anxious at baseline and do not rely on others to meet their needs. Children with disorganized attachment styles are usually angry, non-responsive, passive, depressed, and/or have no coping strategies for how to get their needs met. These attachment styles are believed to shape personality and last into adulthood because of an internal working model mechanism wherein feelings about experiences with the primary caregiver during the first two years of life are stored in the infant's mind and used to predict, interpret, and plan for future events (Bowlby, 1979).

Attachment style and time perspective. Attachment style has also been significantly correlated with time perspective (Laghi et al., 2009) in that: (1) past-negative had a moderate negative correlation with parent attachment; (2) past-negative had a significant negative correlation with peer attachment; (3) hedonistic-present had a significant positive correlation with secure attachment to parents and peers; (4) past-positive had a moderate positive correlation with parent attachment; (5) past-positive had a significant positive correlation with peer attachment; (6) future orientation had a moderate positive correlation with parent attachment; and (7) future orientation had a significant positive correlation with peer attachment amongst adolescents. Time perception was also found to predict identify formation (Luyckx et al., 2010). The findings were varied in the Luyckx et al.'s (2010) study: (1) future time orientation predicted a mild negative relationship with a diffuse-avoidant style and a moderate positive relationship with information-oriented style; (2) present-hedonistic orientation predicted a moderate positive relationship with a normative style; and (3) present-fatalistic orientation predicted a moderate positive relationship with a diffuse-avoidant style. However, past-positive and past-negative time orientations were not considered.

Time perspective was also found to affect the relationship between attachment and resilience. For example, the Aronowitz et al. (2004) study found that future time perspective specifically mediated the relationship. Although there was no direct relationship between attachment and resilience to risky behavior in other time perspectives, maternal caring correlated with attitudes about the future and future aspirations. There was, however, a significant correlation between future aspirations with risky behavior, substance abuse, and violent behavior. These findings lend support to the notion that significant others' future time perspective may also be influential on individual behavior. Resilience findings also indicated a relationship with time perspective.

\section{Resilience}

Resilience is the process by which an individual interacts (copes) with the environment that includes factors which promote well-being and include healthy risks (Zautra et al., 2010). People who are resilient respond to life's challenges with emotional strength and courage instead of experiencing immobilizing defeat or feeling overwhelmed and this ability sets them apart from others (Wagnild, 2011). According to Wagnild (2011), resilient individuals tend to be self-confident and have insight into their abilities and strengths. Resilient 
people face the same stressors as everyone else but they "bounce back" from difficult situations to regain their equilibrium so they can continue with their goals as stated by Wagnild (2011). Through research, Wagnild (2011) found that resilience reversed and protected against feelings of helplessness, fear, anxiety, depression, and other negative feelings and their physical effects (i.e., individuals who were less resilient developed psychiatric disorders such as depression and post-traumatic stress disorder). The model of resilience, which is currently accepted, integrates emotional, biological, and psychological processes as stated by Wagnild (2011).

Resilience can be measured (Wagnild \& Collins, 2009) and has been found to be affected by several variables. There was a positive strong association with both age and health: the older the individual and the healthier the individual, the greater the resilience and the less depression (Wagnild, 2011). Wagnild also reported a strong negative relationship between resilience and depression and females scored significantly higher than males on the 14 item Resiliency Scale she developed (Wagnild \& Collins, 2009).

A resilient student is one who can overcome stressful or challenging barriers to learning in order to achieve academic success (Sarwar, Inamullah, Khan, \& Anwar, 2010; Uzma, 2007). A student who is resilient may overcome challenges to learning in an academic setting through perseverance, having a defined purpose in life including the goal of completing academic work, self-reliance, the ability to comfortably be alone, and equanimity (a balance between positive and negative thoughts and emotions), which are all part of the core of resilience (Wagnild, 2011). Applying Wagnild's (2011) resilience core model to students suggests that how well a student can deal with adverse situations that may affect his or her academic standing may depend on how strong the student's core resilience is. If the resilience core is strong, then the student may not only be able to deal with adversity but also learn from it and apply this learning to difficult situations in the future. A student with a weak resilience core may only be able to maintain status quo at best or may end up giving up due to feeling overwhelmed, in despair, and/or exhausted. The following sections discuss the relationship of resilience with academic achievement and time perspective.

Although it has not been established that resilience may predict time perspective, the opposite relationship exists in that: (1) a present time perspective predicted a significant negative relationship with psychological functioning and well-being (Utsey, Hook, Fischer, \& Belvet, 2008); (2) a past time perspective significantly predicted poor resilience (Fortunato \& Furey, 2010); and (3) future in combination with present time orientation significantly predicted a positive relationship with resilience. Resilience also correlated with time perspective in that: (1) a future time perspective showed a moderate positive correlation; (2) a past time perspective showed a significant negative correlation; and (3) a present time orientation showed a moderate positive relationship (Fortunato \& Furey, 2010).

Not only does time perspective predict resilience, findings suggest that it also has a mediating effect in that neighborhood social conditions predicted time perspective, which then predicted adaptation (Kruger et al., 2008). Specifically, the Kruger et al.'s (2008) study findings also revealed that: (1) high future orientation mediated the relationship between strong positive social attachments and low levels of aggression; (2) low scores on present time perspective mediated the relationship between poor social attachments and high levels of aggression; and (3) low scores on present time perspective mediated the relationship between increased attachment and poor adaptation. Correlations revealed that: (1) the quality of interpersonal relationships had a mild positive correlation with a future orientation; (2) the quality of interpersonal relationships had a mild negative correlation with a present time perspective; (3) resilience had a mild positive correlation with future time orientation; and (4) resilience had a strong negative relationship with present time perception. 


\section{Summary}

The past 30 years of research using Zimbardo and Boyd's $(2008 ; 1999)$ psychology of time theory to predict human behavior has resulted in the belief that the relationship is direct and linear in nature. However, there is evidence that other variables, such as emotional intelligence, psychological resilience, and attachment style may predict time perception. In addition, time perception has been identified as a mediating variable between several behaviors (i.e., social attachment and resilience, self-determination and academic achievement, and belief in a just world and emotional intelligence). The findings have also indicated that time perception continues to develop over time and is flexible in some individuals. This raises the question as to whether these variables operate differently with time perception under structured (coursework phase) verses less structured (dissertation phase) conditions of a distance learning academic setting.

Elements necessary to provide internal structure required for success in academic pursuits include the ability to set and achieve long-term goals through persistence and focus. Academic goal attainment requires giving up immediate gratification for long-term gratification, engaging in school related activities, beginning assignments on time, managing time, utilizing deep-level cognitive processing, persisting in the face of adversity and overcoming barriers, and possessing a sense of self-efficacy. These skills do not develop in present-oriented individuals who doubt they have the capacity to improve their circumstances (Zimbardo \& Boyd, 2008). The coursework phase of doctoral programs may be providing some of these elements through its imposed structure. When some of this structure is removed during the dissertation phase, learners who cannot provide their own structure may precipitate attrition. In addition, findings indicated that other variables such as emotional maturity, resilience, and attachment style might have an influence on time perspective. 


\section{Chapter 3: Research Method}

Zimbardo and Boyd's (1999; 2008) linear unidirectional application of time perspective to academic achievement is limited because of the exclusion of certain variables that may influence the development and maintenance of time perspective and hence, academic achievement. In addition, Zimbaro and Boyd's (1999; 2008) theory claims that time perception continually develops and changes but does not explain which aspects of structure and discipline affect time perspective or its plasticity. Given these gaps in their theory, resilience, emotional maturity, and attachment style were examined for their influence on time perception because of their stated relationships to behavior and time perception in the literature. Yet, the manner in which resilience, emotional maturity, and attachment style may predict time perception is unknown. The purpose of this nonexperimental quantitative survey study is two-fold. First, it was used to identify what predictive role emotional intelligence, attachment style, and resilience (predictor variables) had on time perception (criterion variable) in doctoral distance learners. Second, the study was used to ascertain how emotional intelligence, attachment style, and resilience (predictor variables) interacted to predict time perception (criterion variable). Doctoral distance learners enrolled in online learning programs throughout the United States were invited to complete online questionnaires regarding their time perspective, emotional intelligence, attachment style, resilience, and demographic information.

The following research questions were used to examine the strength of prediction of attachment style, resilience, and emotional intelligence on time perception. The questions were also used to examine how attachment style, resilience, and emotional intelligence interact to predict time perception in doctoral distant learners in the coursework and dissertation phase of their programs. Corresponding null hypotheses and alternative hypotheses are given.

\section{Questions}

Q1: Does resilience predict time perspective in coursework and dissertation phases of a doctoral distance learning program?

Q2: Does attachment style predict time perspective in coursework and dissertation phases of a doctoral distance learning program?

Q3: Does emotional intelligence predict time perspective in coursework and dissertation phases of a doctoral distance learning program?

Q4: How will resilience, attachment style, and emotional intelligence interact to predict time perspective during the coursework and dissertation phases of a doctoral distance learning program?

\section{Hypotheses}

$\mathrm{H1} 1_{0}$ : Resilience will not significantly predict time perspective during the coursework and dissertation phases of a doctoral distance learning program.

H1a: Resilience will significantly predict time perspective during the coursework and dissertation phases of a doctoral distance learning program.

$\mathrm{H} 2_{0}$ : Attachment style will not significantly predict time perspective during the coursework and dissertation phases of a doctoral distance learning program.

H2a: Attachment style will significantly predict time perspective during the coursework and dissertation phases of a doctoral distance learning program. 
$\mathrm{H}_{3}$ : Emotional intelligence will not significantly predict time perspective during the coursework and dissertation phases of a doctoral distance learning program.

H3a: Emotional intelligence will significantly predict time perspective during the coursework and dissertation phases of a doctoral distance learning program.

$\mathrm{H}_{0}$ : Resilience, attachment style, and emotional intelligence will not significantly interact to predict time perspective during the coursework and dissertation phases of a doctoral distance learning program.

H4a: Resilience, attachment style, and emotional intelligence will significantly interact to predict time perspective during the coursework and dissertation phases of a doctoral distance learning program.

Chapter 3 articulates the research methods and design and describes the characteristics and selection of participants, survey instruments, and operational definitions of variables. Data collection, processing, and analysis are described and a discussion of assumptions about the population and design ensues. The study's limitations and delimitations are identified and steps to assure compliance with standards for conducting ethically sound research are stated. The chapter ends with a summation of the research methods and design.

\section{Research Methods and Design}

A nonexperimental quantitative survey design with cross-sectional data collection was used in this study. The design of this study was based on others in the literature that have used a nonexperiemental, quantitative, cross-sectional, online survey design, which has been the gold standard used to identify relationships between time perspective and human behavior (Harber et al., 2003; Horstmanshof \& Zimitat, 2007; Milfont et al., 2008; Zimbardo, 2007; Zimbardo \& Boyd, 1999) and time perspective predictors of human behavior (Barber et al., 2009; Dunkel \& Weber, 2010; Xie et al., 2010; Zimbardo \& Boyd, 1999). A nonexperimental research design was appropriate in order to obtain high quality answers to this study's research questions. This design allowed this researcher to identify the predictive nature of emotional intelligence, resilience, and attachment style on time perspective and to examine differences in this predictive nature during high and low levels of structure in doctoral distance online learners.

Data was collected in order to determine each participant's level of emotional intelligence, psychological resilience, and attachment. Each survey instrument that was used to collect this data has proven reliability and validity ((ZTPI: Horstmanshof \& Zimitat, 2007; Zimbardo \& Boyd, 1999) (RS: Nishi et al., 2010; Wagnild, 2011; Wagnild \& Young, 2009) (PMI: Greenberger et al., 1974) and (RAAS: Collins \& Read, 1990)). Most of these instruments were currently available online via their authors' websites for the purpose of collecting ongoing experimental data for the authors' research; so, these instruments lend themselves well to cross-sectional, online, survey data collection. The ZTPI has been used throughout the literature in seminal studies that examined the relationship between time perception and various human behaviors (Apostolidis \& Fieulaine, 2004; Corral-Verdugo et al., 2006; D’Alessio et al., 2003; Díaz-Morales, 2006; Harber et al., 2003; Horstmanshof \& Zimitat, 2007; Milfont et al., 2008; Zimbardo \& Boyd, 1999). Experimental intervention was not appropriate for this study; however, this study's results may identify and direct the development of interventions designed to enhance students' future orientations so they may be better equipped to complete their doctoral online distance learning program.

In order to investigate the purpose of this study, a research design that allowed the researcher to gather and analyze quantitative data was necessary (Creswell \& Plano Clark, 2011; Teddlie \& Tashakkori, 2009). A quantitative design allowed this researcher to generate numeric data necessary to deductively examine the 
stated predictions using multiple linear regression analysis. Specifically, this research design was used to identify what predictive role emotional intelligence, attachment style, and resilience had on current time perception. This research design was also used to identify how emotional intelligence, attachment style, and resilience interacted to predict time perception in order to expand Zimbardo and Boyd's $(2008 ; 1999)$ linear theory of time perception with respect to the level of structure in distance doctoral programs.

A convenience sample was used by this researcher in order to collect data in the most effective, efficient, inexpensive, and convenient manner in order to obtain the most participants. A convenience sample means that participants from the population will all be invited to participate in this study if they meet the inclusionary criteria. A convenience sample is non-probabilistic in nature, meaning that few efforts will be taken to assure that the sample reflects an accurate representation of the population (Trochim \& Donnelly, 2008). Bias can occur if the sample does not accurately reflect the population, according to Trochim and Donnelly (2008). The sample in this study was gathered using different methods (Department Chair's email to students, online professional and social networking groups, and professor's email to their students) to help decrease bias introduced by the convenience sampling technique. The fact that these methods for gathering participants are all online should not introduce bias since members of the population to be approached are online learners. Trochim and Donnelly (2008) noted that the results of a study in which researchers used a convenience sample cannot be generalized to a general population. Instead, the results generated from a study in which a convenience sample was employed can be used to determine relationships between variables and possibly compared to individuals within the same population if the sample is found to be representative of the population, according to Trochim and Donnelly (2008). In this study, the results cannot be generalized to all distance learning students but must be kept in context of online distance doctoral learners working on or towards a dissertation in the US during a time period close to the time period when the data will be collected.

A cross-sectional, web-based, self-report, survey design was appropriate for this study because attachment style, time perspective, resilience, and emotional intelligence can be reliably measured using standard, valid instruments that have been shown to collect quantitative data obtainable in one cross-section of time. A web-based survey design also allowed this researcher to gather the necessary number of participants from throughout the US at low cost. A survey design posted online via Surveymonkey allowed individuals who meet the inclusionary criteria (students enrolled in an online distance learning doctoral program) to respond if they choose to do so when it is convenient for them. Surveymonkey allowed this researcher to collect data as it was produced by participants in a database set up by the researcher ahead of time. This database was uploaded into SPSS for data analysis making it easy for the researcher to gather and analyze the data.

The data collection for this study was performed once as per a cross-sectional design using a survey. This cross-sectional survey design allowed for the collection and analysis of quantitative data posted by participants in response to the ZTPI (see Appendix A) to measure time perspective, RS (see Appendix B) to assess the level of psychological resilience, PMI (see Appendix C) to test for emotional intelligence, RAAS (see Appendix D) to assess attachment style, and demographic questionnaire (see Appendix E).

Multiple linear regression analysis is a method used to identify the prediction strength of variables and to examine how these predictor variables may work together to affect a criterion variable. Because of its versatility, multiple linear regression analysis enables the investigator to observe the effects of multiple variables or a single variable while eliminating the effects of certain variables as required by the research questions (Agresti \& Franklin, 2013). 
This study planned to use multiple linear regression analysis instead of cluster analysis to test for predictors of time perspective for the following reasons. All of the seminal literature that has examined behaviors predicted by time perspectives has used multiple linear regression analysis and studies that have examined relationships between time perspectives and human behaviors have used correlation analysis. None of these studies have used cluster analysis. The choice to not use cluster analysis may be due to Zimbardo and Boyd's (1999) finding that each of their five time perspectives has the ability to stand alone. A person could be high in all of them at once. Being high in future orientation does not necessarily mean that an individual will be low in present orientation. If a cluster analysis was used to identify different types of time perspective profiles of students, many clusters may result (up to 225) since there are five time perspectives with high, medium, and low scores for each and many different possible combinations of scores for one individual. The use of multiple clusters, even if only a portion were used, might have contaminated this study's results by introducing too many variable combinations. Or, similar results may have been obtained with more effort (e.g., there may be a cluster of students high in future orientation and another high in past-negative orientation, and so forth, which would be similar to the original five time perspectives) along with contamination. Using a cluster analysis would not add any new information to this study that could not be obtained by using multiple linear regression analysis and would increase the needed sample size since it would introduce more variables. Cluster analysis is used when the resulting groups are the matter of interest (Kaufman \& Rousseeuw, 2009). In this study, the resultant groups were not the matter of interest; the interest was in examining the predictive nature of attachment style, emotional intelligence, and resilience on time perspective, and a multiple linear regression would still need to be performed on the various clusters in order to answer the research questions. Furthermore, clinical issues have not typically been addressed using the entire time perspective profile. Instead, efforts have been made to help students develop more of a future orientation in order to perform better in school (Ferrari et al., 2012; Horstmanshof \& Zimitat, 2007). These efforts did not include decreasing students' present-hedonistic natures or past-negative natures, simply future orientation. Instead of working with individuals with post-traumatic stress disorder to increase their future orientation, an effective clinical intervention was to help them to stop dwelling in the past (Zimbardo \& Boyd, 2008).

In this study, multiple linear regression analysis using the Enter method was planned to determine characteristics about the predictive nature of the three predictor variables: attachment style, resilience, and emotional intelligence on the criterion variable of time perspective both individually and in combination in order to answer all of the research questions. The Enter method indicates that predictor variables will be added in the order specified but their order is not an important element of the modeling process (Tranmer \& Elliot, 2008). This method allows all predictor variables to be added to the linear regression equation regardless of their statistical significance, according to Tranmer and Elliot (2008). The Enter method is advantageous over other multiple linear regression models that exclude variables when many predictor variables are used because the other methods eliminate variables based on statistical significance (Tranmer \& Elliot, 2008). Methods that selectively exclude/include predictor variables based on statistical significance include the backward elimination and forward selection models. They are used when many predictor variables are being studied and some have to be included or excluded (Tranmer \& Elliot, 2008). This selection/elimination may cause relevant interactions and important variables to be missed (Tranmer \& Elliot, 2008). Since emotional intelligence, resilience, and attachment style were carefully chosen as possible predictors of time perspective 
based on findings from the literature as described in the "Literature Review" section and since there were only three predictor variables in this study, the Enter method was the method of choice. This research design was not interested in finding the best equation to predict time perspective as would be backward elimination or forward selection because the predictor variables were selected based on a substantive approach that considers the research questions, which were based on past literature. Causation cannot be implied in this research design with a multiple linear regression analysis that utilizes a convenience sample as per Agresti and Franklin (2013).

The philosophical orientation of this research design was based on a postpositivist view. This research study was guided by a postpositivist orientation because it included collecting and analyzing quantitative data on measurable variables using standard, reliable, and valid instruments and statistical analyses, as explained by Teddie and Tashakkori (2009).

\section{Key Elements of the Data Collection Strategy}

The design of this study was used to carry out the following steps, which addressed its quantitative, nonexperimental, cross-sectional characteristics. This researcher added the following standardized assessments: ZTPI (see Appendix A) to measure time perspective, RS (see Appendix B) to assess the level of psychological resilience, PMI (see Appendix C) to test for emotional intelligence, RAAS (see Appendix D) to assess attachment style, and demographic questionnaire (see Appendix E) to the Surveymonkey website in the form of one survey to be taken by willing participants. The survey was posted for three months so participants could complete it at their convenience.

Participants for this web-based, anonymous survey were obtained by the following steps. This researcher approached 25 different online distance learning educational institutions in the US to inquire about their procedure for obtaining research participant students from their doctoral programs. Of the 25 learning institutions, 13 stated that they do not allow outside students to advertise research studies to their students in order to obtain volunteer participants. The remaining 12 learning institutions had policies that allowed outside researchers to request volunteer participants from their doctoral programs. Four out of these 12 learning institutions requested a description of the nature of the research so this researcher sent them the research purpose and design. Upon the receipt of the purpose and design, the four institutions sent their procedures. The other eight institutions sent this researcher their policies without needing to hear the nature of the research. Once Northcentral University's IRB approved this study under the status of "exempt", the approval was sent to all 12 learning institutions in order to obtain permission to obtain research volunteer participants. Four out of the 12 learning institutions granted permission. Each university agreed to send the Invitation to Participate, which described the study (see Appendix F) and included the Surveymonkey link (https://www.surveymonkey. $\mathrm{com} / \mathrm{s} /$ ZHZS3ST) where the survey was posted to their online distance learning students. Follow-up email reminders to disseminate the Invitation to Participate were sent to the appropriate person from the learning intuitions after one month.

In addition to recruiting participants directly through online distance learning institutions, this researcher posted the Invitation to Participate on Twitter, Yahoo, Facebook, and Dissertation Forum after receiving permission to do so by the webmaster of each site's graduate student group. This method allowed for the possibility that the Invitation to Participate would be read by doctoral distance learners who may be appropriate for this study. 
All participants met the inclusionary criteria for this study, which was to be currently enrolled in an online distance learning doctoral program. Participants in the high level of structure group were in the coursework phase of their program while participants in the low level of structure group were in the dissertation phase of their program. Exclusionary criteria included students who were not enrolled in a online distance learning doctoral program (i.e., on leave of absence).

Students who elected to volunteer to participate in this study accessed the Surveymonkey link listed on the Invitation to Participate. After clicking the Surveymonkey link, participants were able to view the Consent Form (see Appendix G), and Cover Letter (see Appendix H), which described the study and indicated informed consent to voluntarily participate in this study. The Consent Form and Cover Letter also notified participants that the survey is anonymous and void of identifying information, their participation is voluntary and they can stop completing the survey at any time. Before proceeding with the survey, participants agreed to take the survey by indicating that they agreed to the conditions above by clicking "continue". The estimated time for completing the survey was approximately 30 minutes. Results were stored by Surveymonkey in their database and then exported to an Excel spreadsheet, then to SPSS for analysis. Participants were not offered any type of incentive to participate in this study.

The final step in the design of this study was to examine all of the research questions using a multiple linear regression with predictor variables of attachment style, emotional intelligence, and resilience and the criterion variable of time perspective utilizing the Enter method. First, each of the predictor variables was analyzed individually with time perspective using Pearson $r$-correlations to determine whether or not there was a statistically significant prediction. Non-significant correlations were eliminated from the multiple linear regression. Then, all three predictor variables were analyzed for their combined effect on the prediction of time perspective. A multiple linear regression was the appropriate method of analysis to use to determine the level of variance in the criterion variables that was due to each predictor variable. A significance level of 0.05 was used to reject the null hypothesis that the fit of the response values predicted by the multiple regression equation for a linear model to what was observed was no different than what would occur by chance. The null hypothesis was also tested by examining $t$-ratios of adding different predictor variables to the multiple linear regression to examine if they do not improve the fit any more than by chance. Two assumptions were violated in the multiple linear regression as explained in Chapter 4, so a step-wise logistic regression analysis was performed and the results of the latter used instead.

\section{Population}

The population targeted in this study was online distance learners enrolled in a doctoral program in the US either in the coursework phase or the dissertation phase of their program. The estimated size of this population is unknown. This researcher has identified 25 different institutions of higher learning in the US that offer online distance Ph.D. programs at the time of this writing. The number of current Ph.D. students in each program of each learning institution is unknown. The closest estimate of the number of online distance learners was made by The Sloan Foundation in its 2011 report, which stated that over 6.1 million students in the US took at least one online course in the fall of 2010 (Allen \& Seaman, 2011). This is a gross overestimate because their report included all higher education online distance learning students, not just doctoral online distance learning students. In addition, not all of the students in their report were enrolled in distance learning programs. Even though this is a gross estimation, the report showed that since 2002, the growth rate of online learners has 
surpassed that of classical learning. In the fall of 2009, the growth rate of online distance learning compared to the previous year was $21 \%$ (972,669 more students) compared to a $2.2 \%$ growth rate in classical higher education during the same time frame according to the same report.

The characteristics of this population are different from that of classical learners. Distance online doctoral students tended to be older and have family and or work responsibilities that keep them from attending classes on campus (Street, 2010). Distance learners were found to be more independent and intrinsically motivated than classical learners who tended to be dependent, extrinsically motivated, and in greater need of interaction with professors and other students (Díaz-Morales, 2006). Adult online distance learners were subject to a higher level of perceived stress than young, unmarried students who attended brick and mortar schools (Ramos, 2011). In addition to the normal stresses associated with demanding graduate programs, online distance learning adults had compounded stress from their outside lives, as explained by Ramos. Yet, there was no difference between online and traditional classroom graduate students in coping with perceived stress.

The population is appropriate to respond to this study's problem and purpose because this study examined the predictive nature of three different variables (emotional intelligence, resilience, and attachment style) on the time perspective of online distance doctoral learners in the US. This population was chosen in order to reach a large number of distance learners from doctoral programs located over a wide geographic area (Gall, Borg, \& Gall, 2007) to meet the sample size requirements (Moore, 2007), which allowed for generalization of results from these participants to other distance doctoral learners. The results of a study conducted to examine time perspective of volunteer participants from US doctoral online programs may provide clarification to Zimbardo and Boyd's (1999; 2008) theory of time and address the high attrition rate of online doctoral programs.

The difference between the population and sample of participants is that the sample consisted of doctoral online distance learners who volunteered through invitation to participate in this study. Participants within the sample self-selected their participation instead of being drawn by this researcher.

\section{Sample}

Participants were 93 distance learning doctoral candidates from universities with an online format, 51 participants were in the coursework phase, 38 participants were in the dissertation phase of their program and four did not indicate which phase of the program they were in. The needed sample size was determined by an a priori power analysis using G*Power 3.1.5 (Faul et al., 2009) using the F-test for linear multiple regression: fixed model, $R$-squared deviation zero with all input parameters: alpha $=0.05$, power $=0.80$, effect size $=0.15$, and number of predictors $=3$.

A convenience sample was utilized meaning that participants from the population were all invited to participate in this study if they meet the inclusionary criteria. This researcher contacted online distance learning institutions that offered doctoral programs in order to secure participants for this study. Each university that agreed to participate in this study agreed to send the Invitation to Participate, which described the study (see Appendix $F$ ) and included the Surveymonkey link (https://www.surveymonkey.com/s/ZHZS3ST) where the survey was posted to their online distance learning students. Follow-up email reminders to disseminate the Invitation to Participate were sent to the appropriate person from the learning intuitions (professor, Chairperson) after one month.

In addition to recruiting participants directly through online distance learning institutions, this researcher posted the Invitation to Participate on Twitter, Yahoo, Facebook, and Dissertation Forum after receiving 
permission to do so by the webmaster of each site's graduate student group. This method allowed for the possibility that the Invitation to Participate would be read by doctoral distance learners who may be appropriate for this study.

Participants met the inclusionary criteria for this study, which was to be currently enrolled in a online distance learning doctoral program. Participants in the high level of structure group were in the coursework phase of their program while participants in the low level of structure group were in the dissertation phase of their program. Exclusionary criteria included students who were not enrolled in an online distance learning doctoral program (i.e., on leave of absence).

Students who elected to volunteer to participate in this study accessed the Surveymonkey link listed on the Invitation to Participate. After clicking the Surveymonkey link, participants viewed the Consent Form (see Appendix G), and Cover Letter (see Appendix H), which described the study and indicated informed consent to voluntarily participate in this study. The Consent Form and Cover Letter also notified participants that the survey is anonymous and void of identifying information, their participation is voluntary, and they can stop completing the survey at any time. Before proceeding with the survey, participants agreed to take the survey by indicating that they agreed to the conditions above by clicking "continue". The estimated time for completing the survey was approximately 30 minutes. Results were stored by Surveymonkey in their database and were exported to SPSS for analysis via an Excel spreadsheet. Participants were not offered any type of incentive to participate in this study.

\section{Materials/Instruments}

A self-report online survey was used for this study. The self-report survey consisted of five published Likert-type scales and a demographic questionnaire (the latter constructed by this researcher) and is described below. These assessments were used to measure time perspective, attachment style, resilience, and emotional intelligence. Their constructs, coding schemes, and psychometric properties are discussed in relation to this study. Reliability coefficient minimal standards of 0.90-0.95 for individual comparisons and 0.70 for group comparisons were used (Wagnild, 2011). Definitions of types of reliability and validity pertinent to all measures in this study were obtained from Wagnild and are as follows. Internal consistency reliability is the extent to which responses are free from random error. This lack of random error implies content homogeneity on multiple items within an assessment and internal consistency among answers to assessment items. Test-retest reliability is the measure of how results obtained from the same individual varies over time. Concurrent validity is a measure of how closely an assessment correlates with other potentially relevant constructs. Known groups validity is the examination of differences between diverse groups of individuals completing the same assessment. Convergent and discriminate validity is the measure of the correlation between two assessments that test for similar constructs. Content validity is a measure of how well an assessment measures what it purports to measure. All instruments have been shown to have acceptable psychometric properties as shown in the sections that follow.

\section{Zimbardo Time Perspective Inventory (ZTPI)}

The ZTPI (Zimbardo \& Boyd, 1999; Appendix A) was used to quantitatively measure the type of perspective each participant had about time on five different time perspectives: past-negative (10 items), past-positive ( 9 items), present-fatalistic (9 items), present-hedonistic (15 items), and future (13 items). The ZTPI is a 56-item self-report assessment that utilizes a 5-point Likert-scale measure which asks participants to 
rate each statement according to how true or characteristic it is of them: 1 ("Very Untrue"), 3 ("Neutral"), and 5 ("Very True"). Sample items include, "Meeting tomorrow's deadlines and doing other necessary work comes before tonight's play", "It doen't make sense to worry about the future, since there is nothing that I can do about it anyway", and "There will always be time to catch up on my work". Some of the items are written in the negative in order to prevent participants from answering compulsively without reading each question carefully. The ZTPI takes approximately 15 minutes to complete.

Results of the ZTPI yield subscores for each time perspective and a total score. The scores on the five different time perspectives are orthogonal in that a subject could score high or low on all five instead of obtaining a percentage on each that would total. For example, present orientation and future orientation are not mutually exclusive. Someone with an orientation towards the future may still enjoy partying all weekend after a very hard week of work and satisfying other obligations (Harber et al., 2003). A person with a present-orientation may force him or herself to complete an overdue assignment instead of engaging in immediate pleasure seeking activities. In order to report the purest results from the ZTPI with regards to time perspective bias, categories of student participants were created that include only extreme scores. For example, a participant was categorized as having a present orientation only if he or she scored in the top quartile of the present-oriented scale and below the top quartile of the future and past-oriented scales. Written permission to use the ZTPI in an online survey was obtained by this researcher (see Appendix I).

There has been over 30 years of research dedicated to the investigation of time perspective using the ZTPI, some of which has examined its psychometric properties. The ZTPI has satisfactory internal consistency as shown by Chronbach's alfa coefficients ranging from 0.74 to 0.83 for the five different time perspectives (Zimbardo \& Boyd, 1999): specifically, future (0.80), past-negative $(0.84)$, past positive (0.80), present-hedonistic (0.77), and present-fatalistic (0.74) (Horstmanshof \& Zimitat, 2007). Test-retest reliability coefficients of the ZTPI have ranged from 0.70 to 0.80 in college psychology majors over a four-week interval as assessed by Zimbardo and Boyd. The ZTPI's structural validity was established using exploratory and confirmatory factor analyses by Zimbardo and Boyd who also found interscale correlations to be low in general $(|0.09| \leq r \leq|0.38|)$. The ZTPI has demonstrated construct and predictive validity. There were correlations between all five time perspectives and 17 indexes of personality, temperament, and coping in the hypothesized direction according to Zimbardo and Boyd. Alcohol and drug use, risk taking, participating in unsafe sex, and neglect of health maintenance was strongly and positively correlated with a present-hedonistic bias of time perception (Keough et al., 1999). Depression, aggression, trait anxiety, and self-esteem correlated with the five time perspectives in the hypothesized direction according to Zimbardo and Boyd (1999). The ZTPI is in public domain and can be found online at Zimbardo's website (http://www.thetimeparadox.com/zimbardo-time-perspective-inventory), in Zimbardo and Boyd's seminal publication (1999), and in Zimbardo and Boyd's book (2008). These studies demonstrate that the ZTPI has acceptable reliability and validity and are appropriate to use for this study.

The ZTPI has several limitations. First, it does not measure the flexibility between time perspective biases (i.e., how easily can an individual switch from a past orientation to a present orientation or to a future orientation as called for in any given situation). This current study did not invest in measuring flexibility between time perspectives. Second, the ZTPI does not include a present orientation that addresses mindfulness (Vowinckel, 2012). While mindfulness may be important to doctoral students, there is currently no assessment of time perspective that includes mindfulness. Third, the ZTPI's measure of the future does not include a 
"future negative" factor. Instead, its measure of future time perspective is a positive one. There is a new assessment that includes a future-negative subscale (Carelli et al., 2011), which has shown initial acceptable reliability and validity by its authors, but was not used in this study because it lacks proven reliability and validity over time. Fourth, the ZTPI also does not account for individuals who act a certain way in order to have a better situation after they die. Finally, some of the ZTPI subscales may measure more than time attitudes (Worrell \& Mello, 2009). This current study was not worried about additional factors that may be contributing to the measurement of time attitudes because the subjective experience of time is complicated and may include multiple variables that if controlled for, may alter the construct the way it was defined by Zimbardo and Boyd (1999; 2008) who clearly delineated many correlations between each time perspective subscale and other variables (i.e., depression, anxiety, impulsivity, and so forth).

\section{The 14-Item Resilience Scale (RS)}

The RS (Wagnild \& Young, 2009; Appendix B), a 14-item Likert-scale assessment, was used to measure resilience in five characteristics of resilience: existential aloneness, self-reliance, perseverance, purposeful life, and equanimity; the scale also yields a total score. According to Wagnild and Young (2009), existential aloneness is the ability to be with one's self because of a well-developed sense of self-worth, self-acceptance, and self-awareness that allows a person to be comfortable being alone. Self-reliance is based on a clear understanding of one's limitations and strengths through various life experiences that teach problem-solving, adaptation, and confidence in order to be able to rely on one's self in order to get through difficult situations. Perseverance is the ability to keep going despite disappointment, discouragement, and difficulties. A purposeful life refers to having found meaning in life that keeps an individual motivated to continue on a path or a goal despite difficulties. Equanimity refers to avoiding extreme negative responses to life situations so that harmony and balance can be achieved leading to an overall sense of optimism and humor. The RS was modified from the 25-item Resilience Scale, which was modified from the original 50-item Resilience Scale developed by the same authors for a population of older women experiencing loss.

When completing the RS, participants in this study were asked to read each of the 14 statements and circle a number ranging from 1 ("Strongly Disagree") to 7 ("Strongly Agree") that best indicated their feelings about each. Participants were asked to respond to all statements. Items include: "I usually manage one way or another", "I can get through difficult times because I've experienced difficulty before", and "My belief in myself gets me through hard times". The higher the score on each of the five characteristics of resilience, the higher the measure of the characteristic (e.g., perseverance). All statements are positively worded. The higher the overall score, the higher the resilience. The RS can be completed in less than four minutes.

The RS has the following psychometric properties demonstrating strong reliability and supported validity. In developing the 14-item RS from the 25-item Resiliency Scale, 14 items were selected that had above 0.04 on inter-item correlations (range 0.41-0.65) and that represented the five resiliency core components. The 14-item $\mathrm{RS}$ was found to be strongly correlated with the 25 -item Resiliency Scale $(r=0.97, p<0.001)$. The internal consistency reliability for the RS ranged from a coefficient alpha of 0.91 o 0.94 (Nishi et al., 2010). The specific Cronbach's alpha coefficients were found to be 0.92 for a depressed sample $(n=63), 0.93$ for a healthy sample $(n=707), 0.94$ for a sample of people with heart disease $(n=111)$ (Wagnild, 2011), and 0.94 for older gay men $(n=260)$ (King, 2009). The RS had a test-retest reliability of 0.91 (Wagnild \& Young, 2009). Concurrent validity was measured by correlating the RS with life satisfaction, morale, and depression (Nishi et 
al., 2010). Nishi demonstrated a positive moderate relationship between resilience and life satisfaction and resilience and morale and a moderate negative correlation between resilience and depression. Multiple regression analysis demonstrated that the better the health, the greater the age, and the less depression reported, the greater the overall score on the RS (Wagnild, 2011). Scores on the RS were lower for bipolar individuals ( $n$ $=28)$ and individuals with depression $(n=77)$ and higher for individuals who were healthy $(n=343)$ demonstrating expected results for known groups validity (Wagnild, 2011).

The RS demonstrated strong convergent and discriminate validity. The total RS score showed a strong positive correlation with the following subsets of the Health Promoting Lifestyle Profile (a measure of behaviors that promote health with proven validity and reliability) in a sample of adults $(n=776)$ : stress management, interpersonal support, and self-actualization and a low correlation with exercise and nutrition as was hypothesized (Wagnild, 2011).

Permission to use the RS in this online research survey study was requested via email to the author who responded by giving purchasing and licensing information on this website (www.resiliencescale.com). This researcher purchased the manual (Wagnild, 2011) and obtained the required Intellectual Property License Agreement for Students (see Appendix J), which allowed this researcher to measure resilience in an unlimited number of subjects effective on July 26, 2013 for one year at which time it can be renewed.

\section{The Psychosocial Maturity Inventory (PMI)}

The PMI (Greenberger et al., 1974; Appendix C) was used to measure emotional intelligence. This 30-item, reverse-scored, 4-point Likert-scale evaluates personal responsibility. The PMI has nine subscales: Self-Reliance, Work Orientation, Identity, Communication Skills, Roles, Social Commitment, Tolerance, Openness to Change, and Enlightened Trust but only three will be used (Self-Reliance, Work Orientation, and Identity) to give one summary score labeled Individual Adequacy. The use of these three subscales instead of all nine subscales still preserves the construct of emotional intelligence and the psychometric properties of the assessment according to Greenberger et al. (1974). When completing the PMI, participants in this study were asked to read each of the 18 statements and circle a number ranging from 1 ("Strongly Disagree") to 4 ("Strongly Agree") that best indicates their feelings about each. Participants were asked to respond to all statements. Sample items include, "Why work for something others will enjoy if you won't be alive to enjoy it too?" (Social Commitment), "I feel a little sorry for people whose ideas about God are different from mine" (Tolerance), and "If everyone is to be really equal, some people will have fewer advantages than they have now" (Openness to Change). The overall psychosocial maturity score for Individual Adequacy was derived from the mean across all 18 statements.

The PMI has excellent reliability with a Chronbach's alpha coefficient of 0.89 and an adequate fit to baseline data (NFI $=0.82, \mathrm{CFI}=0.87$, RMSEA $=0.04$; Greenberger et al., 1974). The PMI has been published by Greenberger et al. (1974), so is in the public domain. Permission to use the PMI in this online survey study was obtained by this researcher (see Appendix K).

\section{The Revised Adult Attachment Scale (RAAS)}

The RAAS (Collins \& Read, 1990; Appendix D) was used to measure participants' attachment style quantitatively. The RAAS has three subscales: Close, Anxious, and Dependent. The Close subscale measures the degree to which participants feel comfortable with closeness in relationships. The Depend subscale measures the degree to which participants believe they can depend on others. The Anxiety subscale measured fear of abandonment. The RAAS subscales correspond to the attachment styles of Secure, Avoidant, 
Anxious-Ambivalent (Anxious-Secure and Anxious-Avoidant) as reported by Collins and Read (1990) and described below. Individuals with secure attachment styles scored high on the Depend and Close subscales and low on Anxiety. The anxious-ambivalent attachment style corresponded to individuals who scored high on Anxiety and moderate on Close and Depend. Individuals with avoidant attachment styles scored low on all three subscales.

The RAAS is an 18-item, self-report, 5-point Likert scale, which includes items such as, "I find it difficult to allow myself to depend on others" (Depend), "I find others are reluctant to get as close as I would like" (Anxiety), and "I find it relatively easy to get close to others" (Close). When completing the RAAS, participants in this study were asked to read each of the 18 statements and circle a number ranging from 1 ("Not at all Characteristic") to 5 ("Very Characteristic") that best indicates their feelings about each item. Participants were asked to respond to all statements.

The RAAS has demonstrated acceptable reliability and validity. The RAAS has internal consistency of 0.75 for Depend, 0.72 for Anxiety, and 0.69 for Close items and test-retest reliability of $0.71,0.52$, and 0.68 respectively as measured by Cronbach's alpha coefficient (Collins \& Read, 1990). The RAAS has been validated by a factor analysis that supported the three subscales as reported by Collins and Read (1990). The RAAS has been used by Collins and Read (1990) to study the relationship between attachment and trust, self-esteem, love, child-parent relationships, and social self-perception as described below. Participants who scored high on Depend and Close subscales tended to report higher self-esteem, a better sense of being in control of their lives, being more independent, being more trusting when compared to participants who scored high on Anxiety. Also in contrast to participants who scored high on Anxiety, those who scored high in Depend and Close reported warm memories of their parents who were experienced as non-rejecting.

Prediction studies have been performed using the RAAS by Collins and Read (1990) and are described below. The opposite sex parent's attachment style predicted the attachment style of the current love object. Scores on the Close Subscale predicted (in a positive direction) satisfaction of the significant love object: (1) high Close scores were characteristic of women who experienced their male lovers as more warm, trustworthy, self-disclosing, communicative, and responsive as compared to the other subscale factors; and (2) men who had high Close scores tended to believe that their female partners were more satisfied with their relationship. Anxiety and Depend had few correlations with relationship assessment. Their study showed that individuals tended to pick partners who reinforced their relationship expectations: men afraid of abandonment chose women who disclosed less, reinforcing their fear of abandonment.

A limitation of the RAAS is that participants who take it may respond to its statements as if describing their current relationship and not all of their relationships. If answered in this manner, the RAAS may be measuring a state, not an enduring trait. Other studies are necessary to confirm the relationship between the RAAS' three factor domains and the three attachment styles of Secure, Avoidant, Anxious-Ambivalent (Anxious-Secure and Anxious-Avoidant). The RAAS has been published by Collins and Read (1990), so is in the public domain. Permission to use the RAAS in this online survey study was obtained by this researcher (see Appendix L).

\section{Demographic Questionnaire}

Demographic data were collected via a questionnaire (Appendix E) constructed by this researcher; items included age, gender, income, and phase of study (coursework versus dissertation). These data were used to 
identify which participants were in which level of structure group: coursework phase (high amount of structure) or the dissertation phase (low amount of structure). The demographic data were also used for statistical control.

\section{Operational Definition of Variables}

The predictor variables for this study were emotional intelligence, attachment style, and resilience. The criterion variable was time perception. Demographics included age, gender, and income.

Age. Age was measured in years (ratio data) from the date of birth until the time of completion of the demographic questionnaire. Mean ages for the two different groups: coursework phase and dissertation phase was obtained from the Demographic Questionnaire (see Appendix F).

Attachment style. Attachment style was a predictor variable that was measured using a self-report 5-point Likert scale, which addressed respondents' feelings and perceived functioning in romantic relationships where 1 ("Not at all Characteristic") through 5 ("Very Characteristic") produce ordinal data. Median scores were derived from the RAAS (Collins \& Read, 1990; see Appendix E).

Emotional intelligence. Emotional intelligence was a predictor variable that was measured using a self-report 4-point Likert scale, which addressed respondents' sense of personal responsibility ranging from "Strongly Disagree" to "Strongly Agree" producing ordinal data. Median scores were derived from the PMI (Greenberger et al., 1974; see Appendix D).

Gender. Gender was a dichotomous nominal demographic measured by assigning the numbers 1 (male) and 2 (female). Descriptive statistics of male and female participants across groups were calculated from data obtained from the Demographic Questionnaire (see Appendix F).

Income. Income was a demographic measured for annual salary (ratio data; range $\$ 0$-infinity) accrued by the participant in the year prior to taking the survey as self-reported on the Demographic Questionnaire (see Appendix F).

Level of structured time. Level of structured time was a construct defined by two levels: the coursework phase (highly structured) and dissertation phase (less structured) of a distance learning doctoral program. Level of structured time was a binary variable such that the coursework phase $=0$ and dissertation phase $=1$. During the coursework phase, syllabi provide structured assignments with due dates, grading rubrics, academic standards, and required reading/resource material. A learner profile reminds learners of which and how many courses they have taken and still need to take and calculates the GPA. Cumulatively, the effect of these elements provides such a highly structured learning environment that students are required to create fewer of these structures/elements themselves.

During the dissertation phase, some of the structure provided in the coursework phase decreases. The dissertation process is much longer than 12 weeks and is guided by more than one professor, each having to come to agreements when reviewing the dissertation. The process of completing the dissertation requires more independence of the learner in the form of tenacity, better time management, a higher degree of personal causation, strong executive functioning skills, good frustration tolerance, and adequate ego control. The phase of the doctoral program was determined through the Demographic Questionnaire (see Appendix F) administered to each participant.

Resilience. Resilience was a predictor variable that was measured using a self-report 7-point Likert scale, which addressed respondents' psychological resiliency ranging from 1 ("Agree") to 7 ("Disagree") producing ordinal data. Median scores were derived from the RS (Wagnild \& Young, 2009; see Appendix B). 
Time perspective. The process through which individuals make sense of personal events and sort them into time zones or temporal categories has been defined as "time perspective" (Zimbardo \& Boyd, 2008). In contrast to objective or clock time, time perspective emphasizes subjective and inter-subjective experiences of time. Zimbardo and Boyd (2008) identified and defined five different categories that comprise the construct of time perspective (see Definition of Terms section) and served as criterion variables in this proposed study.

The ZTPI (Zimbardo \& Boyd, 1999; see Appendix A) is a 56-item self-report 5-point Likert scale that obtains ordinal data for past-positive, past-negative, present hedonistic, present-fatalistic, and future time perspectives. Each time perspective generated a median score based on the number of test items in each time perspective category: past-negative has 10 items, present-hedonistic has 15 , future has 13 , and past-positive and present-fatalistic have nine. A median score of 1 is considered the lowest score for a time perspective, 2 is low, 3 is neutral, 4 is high, and a median score of 5 is the highest. The higher the score, the more characteristic the participant is of that particular time perspective given that participants can have a mixture of time perspectives and each is unique. Zimbardo and Boyd (2008) reported the ideal time-perspective profile to be low in present-fatalistic and past-negative, moderately high in present-hedonistic and future, and high in past-positive.

\section{Data Collection, Processing, and Analysis}

A cross-sectional quantitative design was used in this proposed nonexperimental survey study. The site of the data collection was online universities with doctoral programs that utilize asynchronous distance learning. Each university that agreed to participate in this study agreed to send the Invitation to Participate, which described the study (see Appendix F) and included the Surveymonkey link (https://www.surveymonkey. $\mathrm{com} / \mathrm{s} /$ ZHZS3ST) where the survey was posted to their online distance learning students. Follow-up email reminders to disseminate the Invitation to Participate were sent to the appropriate person from the learning intuitions (professor, Chairperson) after one month.

In addition to recruiting participants directly through online distance learning institutions, this researcher posted the Invitation to Participate on Twitter, Yahoo, Facebook, and Dissertation Forum after receiving permission to do so by the webmaster of each site's graduate student group. This method allowed for the possibility that the Invitation to Participate would be read by doctoral distance learners who may be appropriate for this study.

Within this cluster, students who were in the doctoral phase of the distance learning program identified themselves as such and were distinguished from those in the coursework phase of the program (also self-identified). The population was stratified for gender and age before selection in order to match other populations in the literature.

Data (predominantly ordinal or ratio) were collected from five questionnaires (see Appendices A-E for a copy of each questionnaire). Except for the demographic questionnaire, the other four are standardized and published with proven reliability and validity. The questionnaires were posted online via Surveymonkey (https://www.surveymonkey.com/s/ZHZS3ST). This method of data gathering was used in order to reach a large number of distance learners from doctoral programs located over a wide geographic area (Gall et al., 2007). This method of data collection was necessary in order to meet the sample size requirements (Moore, 2007), which allowed for generalization of results from these participants to other distance doctoral learners. A multidimensional approach to time perception can be achieved via the assessment of ZTPI, which has been used successfully to quantify subjective, inner-subjective, and objective approaches (Zimbardo \& Boyd, 1999). 
Quantitative data were prepared by developing a codebook of variables with definitions and numbers assigned to each to speed the interpretation with accuracy. Continuous data were coded as-is in spreadsheet software. Categorical data such as gender and others were coded in a corresponding numerical form for statistical procedures. After coding all the required data in the spreadsheet software, data were saved in comma separated values format and readily imported into the SPSS Statistics Gradpack version 19.0 (2011) statistical software, which was used for all data analysis. A correlation matrix was used to examine whether the quantitative variables were correlated. In order to report the strength of correlation results, Green and Salkind's (2011) delineation of strength between two variables was used: Pearson Correlation Coefficients of 0.10, 0.30, and 0.50 were interpreted as small, medium, and large, respectively, regardless of direction of the relationship. The way in which a particular discipline interprets the strength of its correlations is specific to each field of study and can vary accordingly. Green and Salkind's (2011) delineations were used to interpret the strength of correlations in this study because they are conventionally used by behavioral scientists. Statistics were displayed for each of the quantitative survey instruments.

The researcher of this study planned to use multiple linear regression analysis. According to Osborne and Waters (2002), there are four main requirements present in multiple linear regression analysis for which violations are not robust. They are normality, linearity, reliability of measurement, and homoscedasticity. This researcher tested for these four and showed the results. ANOVAs were performed to test for normality. Kolmogorov-Smirnov and Shapiro-Wilk tests were used to test for abnormal distributions. Bivariant/multivariate data cleaning was used if abnormal distributions were present. There also needs to be a linear relationship between predictor and criterion variables. If there is not, a Type II error and under-estimation may occur. A Type I error may also occur as an over-estimation for the other criterion variable if it shares variance will the first criterion variable. For some variables, literature has shown relationships to be linear, but results from the literature may not be foolproof. Therefore, tests for linearity were performed. A third requirement of multiple linear regression analysis is that the variables be measured in a reliable fashion. If they are not, an over-estimation can occur in the effect sizes of the other variables. Therefore, low reliability will be corrected for. The fourth requirement is that homoscedasticity exists in that variance of errors is the same for all levels of the criterion variables. If not, heteroscedasticity can result in Type I error. Levene's tests were used to check this requirement.

Since the demographic of gender is categorical and all of the predictor variables are continuous, un-standardized regression coefficients (Schielzeth, 2010) were used to measure the effects of each predictor variable: attachment style, emotional intelligence, and resilience on time perception for each gender separately because, unlike correlations, regression coefficients are not influenced by changes in variance of the predictor variable or differences in measurement error in the criterion variable (Schielzeth, 2010). But first, tests of the difference between regression coefficients were performed (Faul et al., 2009). Because the demographics of income and age and the predictor variables are all continuous and the relationship is thought to be linear in most cases (e.g., as age and income increased, future orientation increased; Padawer et al., 2007), the product of the demographic indicator and the predictor variable was added to the regression equation (Faul et al., 2009). Measurement error in the demographic indicators was remedied by assessing for (Faul et al., 2009) and correcting (Schielzeth, 2010) differences between regression coefficients as above.

A multiple linear regression analysis across all participants was performed using the Enter Method. A covariance matrix, collinearity diagnostics, and part and partial correlations were performed to determine 
whether multicollinearity (Licht, 1995) bias introduced large standard errors in the prediction model. If each predictor variable is highly correlated with the criterion variable, but is minimally correlated with each other, the regression model will be considered robust; however, if multicollinearity bias is indicated, a number of corrections may be made (e.g., standardization of the predictor variables, mean-center the predictor variables, adding or removing variables, regression stepwise method, principal component regression). No significant collinearity existed but two assumptions were violated so the results of logistic regressions were used instead.

\section{Assumptions}

There are several assumptions about the participants of this proposed study. It is assumed that participants were able to read and understand the assessment questions because they met the admission requirements for a Ph.D. program and were actively participating in that program. All of the assessments in this survey have been used extensively with high school and freshmen college students without mention of biasing the results due to misunderstanding the questions (Boyd et al., 2010; Boyd \& Zimbardo, 2005; Harber et al., 2003; Horstmanshoft \& Zimitat, 2007; Zimbardo \& Boyd, 1999). Therefore, misunderstanding of survey questions should not have been a problem of this study that examines doctoral students.

It is also assumed that participants answered the questions honestly and accurately. This is a common assumption of self-report assessments. In this study, there was no pressure for respondents to answer the survey questions any differently than would reflect their normal behavior because their answers were anonymous, no incentives were given to participate, and no consequences took place as a result of their answers. Because participants responded anonymously via the Internet, the impetus to be dishonest to appear more socially desirable should have been minimized. In addition, several of the assessments in the survey, such as the ZTPI, contain questions written in the negative to minimize impulsive or dishonest responses (Zimbardo \& Boyd, 1999). The most accurate assessment of subjective and inter-subjective experiences of time perspective would be generated by participants themselves and not measured by a researcher's interpretation of the participants' experience of time, which would be once removed and less accurate. Self-report measures of adult attachment style, resilience, and emotional intelligence have proven reliability and validity as stated in the Materials/Instruments section of this proposal.

The subjects were assumed to be representative of online, Ph.D. distance learners because they were solicited from different higher educational institutions throughout the US. Applying time perception theories to this population was in keeping with the theoretical frame of reference for this proposal.

\section{Limitations}

The nature of the survey assessments may have posed limitations on this proposed study. All the assessments in the survey were self-report, which can result in a Hawthorne effect as mentioned above. None of the assessments included items to identify social desirability, honesty, or inconsistencies. Even if the participants answered to the best of their ability and as honestly and forthcoming as possible, some participants may have had limited self-awareness and so may have reported inaccurately. Participants were instructed to take the survey at home in a comfortable setting without distraction; however, the assessment-taking environment cannot be controlled. This researcher cannot guarantee that the survey was completed in one sitting by the same responder of sound mind, but was mindful of the delimitations that the assessments may present. This researcher made every attempt to solicit participants from different higher educational institutions 
throughout the US, but if there was a lack of representation from certain geographic locations, there can be no guarantee that the subjects were representative of online, Ph.D. distance learners in the US.

The nature of the participants may also have posed limitations on this study. The voluntary status of the participants could have produced a bias because of different personality characteristics of those who volunteered and those who did not. The demographic characteristics of the participants were unknown at the time of data collection and the population's demographic characteristics will never be known. Most participants were from middle to upper middle-class in their socio-economic status, a demographic that can also introduce a bias. The sample will be obtained through purposeful sampling instead of randomization. The ability to generalize the results of this study is therefore limited. All participants were engaged in higher education, so the effects of education on resilience, time perspective, or the development of emotional intelligence could not be examined in this study.

The nature of the design of this study may also have posed limitations. The researcher of this nonexperimental quantitative survey used correlations to analyze the type and strength of relationships between variables, so no causal relationship could be established between variables. However, multiple regressions can control for possible confounding variables thereby increasing internal validity.

\section{Delimitations}

The following characteristics were selected by this researcher to define the boundaries of this proposed study. Participation in this proposed study was delimited to distance learning Ph.D. students from universities throughout the US with an online learning format. Students enrolled in other doctoral programs that do not require a dissertation, hence do not award a Ph.D. (e.g., physical therapy doctoral programs awarding a physical therapy doctorate or PTD), were excluded. Students enrolled in doctoral programs outside the U.S. were also excluded. This researcher delimited this study to measuring attachment style, resilience, emotional intelligence, and time perception in doctoral distance learners enrolled in either the coursework or the dissertation phase of their program. No other variables were investigated, even though there may be other variables that influenced the perception of time. These variables were measured using specific self-report, Likert scale assessments selected over other assessments because of their proven reliability and validity and predominance in the literature. The results of the proposed study can be generalized only to distance learning Ph.D. students from universities throughout the US with an online learning format.

\section{Ethical Assurances}

This researcher took the following steps to ensure that this study complied with the ethical standards of protection from harm, informed consent, right to privacy, and honesty with professional colleagues. This researcher made every effort to protect all participants from harm during their participation in this study. An application to the Institutional Review Board of Northcentral University was made by this researcher to obtain approval for this non-experimental study before any data was collected. The status granted was "exempt" for two reasons. First, the researcher of this study was not in any way biased toward inclusion of vulnerable population groups such as fetuses, prisoners, children, elders, pregnant woman, females, members of minority groups and their sub-populations, or individuals with disabilities. This researcher made sure that the surveys were equally available to any gender and racial/ethnic/religious group and did not discriminate against people with alternative sexual orientations. Second, this project did not involve invasive procedures or other medical or therapeutic interventions. This researcher did not misinform or mislead participants in any way. No risk to the subjects existed beyond those of everyday living. However, minimal risk to participants may have arisen 
from psychological reactions to certain questions on the survey (Borden \& Abbott, 2005). Participants were granted permission to stop the survey at any point or to skip a question if they were not comfortable answering it. A statement to this effect was included in the Consent Form posted on the first page of the Surveymonkey link (https://www.surveymonkey.com/s/ZHZS3ST) by this researcher. Such individuals had an option of using the researcher's e-mail address that was provided by this researcher on the Informed Consent Form in case concerns arose during the completion of the survey. In addition, a comment section was added to the end of the survey to provide an opportunity for participants to raise issues regarding the study. Participants who wanted more information about time perspectives could read Zimbardo and Boyd's book (2008) that was referenced in the survey. In this book, Zimbardo and Boyd (2008) explain the value of each time perspective so participants who have negative feelings about the survey or their results could gain a more positive understanding of their time perspective. Participants who experienced negative feelings after taking the survey could have contacted this researcher (who has a doctorate in psychoanalysis and is a licensed, certified psychoanalyst) at the contact information listed on the Invitation to Participate and Consent Forms and this researcher would help individuals feel better. Therefore, harm to subjects should be minimal.

All participants provided informed consent via the Consent Form, which included a brief introduction to the study: its purpose, nature, risks, benefits, and participants' status (voluntary). Participant's submission of a survey also indicated consent. The Invitation to Participate flyer (see Appendix F) and the Cover Letter introducing the study (see Appendix H) were sent by this researcher to the chairpersons/directors and also included this information. There was no monetary compensation or incentives for the participants in this study. Therefore, the nature of this study and participants' involvement was clarified so informed consent could be given prior to completion of the survey.

The privacy of all participants was upheld by this researcher. Participants did not give the researcher their names. The surveys contained no questions that ask for identifying information, so no personal data was gathered or shared. The researcher did not know the identity of the participants beyond age, gender, educational level, school, marital status, economic status, ethnicity, and learning environment. Surveymonkey was password-protected for additional security. Surveys were sent from the participants to a Gmail account created by the researcher for storing responses safely, which was accessed by the researcher only and password protected. Each survey was coded by the researcher with an identification number for storing data. Participants may have elected to give their e-mail address to the researcher to receive their scores. Under this condition, only the researcher had access to these e-mail addresses. The researcher coded the e-mails, calculated the scores of each survey, and sent the scores to participants who requested them. When the study was finished, the Gmail account was terminated by the researcher so it could not be accessed afterwards. Results were reported in an aggregated format. All hard copies of data material will be destroyed by a paper shredder and all electronic files will be deleted from the researcher's computer 5 years post completion of the study. By using these methods, participants' anonymity was preserved throughout the duration of this study.

Honesty with professional colleagues was upheld. This researcher presented all of the results in an honest fashion within the dissertation and will do so in any publication that includes its results. No distortion or fabrication of data took place to support a particular hypothesis. Turnitin was used by this researcher and her mentor on the dissertation manuscript to assure that no plagiarism inadvertently occurred. No documentary theft was employed. In this way, the researcher of this study reported honest and original information to the field of psychology. 
Participants could elect to give their email address to the researcher to receive an executive (brief) summary of this study's results. Under this condition, only the researcher had access to these email addresses. This researcher coded the emails, calculated the scores of each survey, and sent the scores to participants who requested them.

Permissions were obtained from copyright holders of all four assessments to use their assessment in this online survey study (Appendixes I-L). Permissions to obtain voluntary research participants were obtained from Walden University (Appendix M), Valdosta State University (Appendix N), Keiser University (Appendix O), and Stony Brook University (Appendix P).

\section{Summary}

By conducting nonexperimental quantitative research in the form of a cross-sectional survey, information on the role of emotional intelligence, attachment style, and resilience on time perception in 93 doctoral distance learners was gathered and analyzed using multiple linear regression analysis. Results provided clarification to Zimbardo and Boyd's theory of time $(1999 ; 2008)$ by uncovering the predictive role that these three predictor variables had on time perception during a structured phase (coursework phase) and the less structured phase (dissertation phase) of doctoral distance learning. 


\section{Chapter 4: Findings}

The purpose of this nonexperimental quantitative survey study was two-fold. First, it was used to identify what predictive role emotional intelligence, attachment style, and resilience (predictor variables) had on time perception (criterion variable) in doctoral distance learners. Second, the study was used to ascertain how emotional intelligence, attachment style, and resilience (predictor variables) interacted to predict time perception (criterion variable). Participants were 93 distance learning doctoral candidates from universities throughout the United States with an online format, 51 participants were in the coursework phase, 38 participants were in the dissertation phase of their program, and four did not indicate which phase of the program they were in. They were recruited from various universities and professional doctoral forums through the internet. Survey data were collected using Surveymonkey between October 2 and December 31, 2013. Questions regarding demographics, time perception, personal relationships, resilience, and emotional intelligence were asked.

Chapter 4 presents the findings of this study. Descriptive statistics (frequencies, percentages) are given for demographics (gender, income, age, marital status, race, and program phase). Means and standard deviations are reported for the criterion (time perspective) and predictor (attachment style, resilience, and emotional intelligence) variables. Correlations between predictor and criterion variables and between age and the variables mentioned above are reported.

The results of the tests for assumptions revealed that there were two violations of multiple linear regression analysis: normality and homoscedasticity. Therefore, results of step-wise logistic regression analyses are presented instead of multiple linear regression analyses since there are fewer assumptions for the former type of statistical analysis, and most assumptions were metor corrected for in order to run a step-wise logistical regression. The results of the logistic regressions revealed the predictive nature of attachment style, resilience, and emotional intelligence on time perspective in course work and dissertation phases of online distance doctoral learners and are presented in this chapter.

In addition to the statistical results mentioned above, this chapter also includes a discussion of assumptions for all statistical analyses and identifies any violations of these assumptions. Finally, an evaluation of the findings is included.

\section{Results}

The results section includes a report of descriptive statistics, which includes frequencies, means, and standard deviations; correlations; analysis of research questions; and assumptions and violations. There were only a few missing observations and they were list wise deleted.

Descriptive statistics. A report of frequencies of the demographic variables and program phase is summarized below. An analysis of descriptive statistics, including minimum and maximum values, means, and standard deviations can be found in Table 1 and is summarized below.

Frequencies. The total number of participants that responded to the online survey was 93 . Of these 93,68 or $73.9 \%$ were female and 24 or $26.1 \%$ were male. The six groups of annual income reported via the demographic questionnaire were collapsed into three groups post hoc for ease of analysis. These three groups were: $\$ 0-\$ 40,000$ characteristic of 21 or $22.8 \%$ of the participants, $\$ 41,000-\$ 80,000$ characteristic of 28 or $30.4 \%$ of the participants, and above $\$ 80,000$ characteristic of 43 or $46.7 \%$ of the participants. Participants ranged in 
age from 24 years to 67 years with a mean of 45.18. Fifty-seven of the participants were married (62.6\%), while 18 or $19.8 \%$ were single and 16 or $17.6 \%$ were divorced. The five survey categories for race were collapsed into three post hoc for ease of analysis. Seventy-two percent or 67 of the participants were White, $16.1 \%$ or 15 were African American, and $11.8 \%$ or 11 were Other/unknown. Fifty-one or $57.3 \%$ were in the course work phase of their online distance learning doctoral program and 38 or $42.7 \%$ were in the dissertation phase of their online distance learning doctoral program.

Means and standard deviations. Descriptive statistics for scales and subscales are given in Table 1, which includes the $N$, minimum score, maximum score, means, and standard deviations.

Table 1

Descriptive Statistics

\begin{tabular}{llccrc}
\hline Variable & $N$ & Min & Max & Mean & $S D$ \\
\hline Age & 91 & 24 & 67 & 45.18 & 10.00 \\
Past-negative & 85 & 1.70 & 4.60 & 2.97 & 0.67 \\
Past-positive & 85 & 1.78 & 4.67 & 3.54 & 0.61 \\
Present-hedonistic & 85 & 2.14 & 3.93 & 3.02 & 0.43 \\
Present-fatalistic & 85 & 1.13 & 3.14 & 2.19 & 0.52 \\
Future & 85 & 2.83 & 5.00 & 3.96 & 0.46 \\
Resilience & 85 & 63.00 & 98.00 & 86.04 & 8.13 \\
Emotional Intelligence & 84 & 7.67 & 12.00 & 10.26 & 0.96 \\
Dependent Attachment Style & 84 & 1.00 & 5.00 & 2.93 & 0.93 \\
Anxiety Attachment Style & 84 & 1.00 & 4.50 & 1.84 & 0.72 \\
Close Attachment Style & 85 & 1.00 & 5.00 & 3.53 & 0.79 \\
\hline
\end{tabular}

Correlations. Pearson correlations with 2-tailed significance with an alpha significance level of 0.05 were done between the past-negative subscale, past-positive subscale, present-hedonistic subscale, present-fatalistic subscale, future subscale, resilience scale, PMI final score (a measure of emotional intelligence), RAAS dependent subscale of attachment style, RAAS anxiety subscale of attachment style, RAAS close subscale of attachment style and age. A correlation matrix of the relationship between these variables can be found in Table 2. The correlations that are significant with a $p$-value $<=0.05$ are as follows.

All time perspective subscales had significant correlations with many of the predictor variables except for future orientation. Past-negative showed a moderate negative correlation with resilience $r(85)=-0.39, p=$ 0.001 , emotional intelligence $r(84)=-0.46, p=0.001$, RAAS dependent $r(84)=-0.47, p=0.001$, and RAAS close $r(84)=-0.35, p=0.001$. Past-negative had a moderate positive correlation with RAAS anxiety $r(84)=$ $0.30, p=0.005$. Past-positive demonstrated a moderate positive relationship to RAAS dependent $r(84)=0.33$, $p=0.003$. Past-positive had a weak negative correlation with RAAS anxiety $r(84)=-0.26, p=0.018$. Past-positive had a weak positive correlation with RAAS close $r(85)=0.24, p=0.027$. Present-hedonistic demonstrated a weak negative correlation with emotional intelligence $r(84)=-0.27, p=0.027$. Present-hedonistic had a weak positive correlation with RAAS anxiety $r(84)=0.27, p=0.012$. Present-fatalistic showed a moderate negative correlation with emotional intelligence $r(84)=-0.33, p=0.002$. Present-fatalistic demonstrated a weak positive correlation with RAAS anxiety $r(84)=0.28, p=0.009$.

Although the correlations reported above were significant, there were a number of correlations that were not significant between the predictor variables and criterion variable subscales (see Table 2), possibly indicating a violation of the assumption of multiple linear regression that requires linearity. The assumption of 
linearity for a multiple linear regression analysis also includes non-significant relationships between all predictor variables. In this case, five relationships may be potential violators of the assumption of independent predictor variables (see Table 3). Resilience had a strong positive correlation to emotional intelligence $r(84)=$ $0.60, p=0.001$. Resilience had a moderate positive correlation with RAAS close $r(85)=0.32, p=0.003$. Emotional intelligence correlated with all three subscale of attachment style. The correlation with the RAAS close subscale was moderate and positive $r(84)=0.45, p=0.001$. The correlation with the RAAS anxious subscale was weak and negative $r(84)=-0.24, p=0.031$. The correlation with the RAAS dependent subscale was moderate and positive $r(84)=0.31, p=0.004$.

Table 2

Summary of 2-tailed Pearson Inter-correlations for Scores on Measures of Criterion and Predictor Variables and Age

\begin{tabular}{|c|c|c|c|c|c|c|c|c|c|c|c|}
\hline & PN & PP & $\mathrm{PH}$ & PF & $\mathrm{F}$ & $\mathrm{R}$ & EI & Dep & Anx & Close & Age \\
\hline \multicolumn{12}{|l|}{ PN } \\
\hline PP & $-0.36^{* *}$ & & & & & & & & & & \\
\hline PH & 0.17 & 0.02 & & & & & & & & & \\
\hline PF & 0.16 & 0.02 & $0.46^{* *}$ & & & & & & & & \\
\hline $\mathrm{F}$ & 0.01 & -0.010 & $-0.47^{* *}$ & $-0.39^{* *}$ & & & & & & & \\
\hline $\mathrm{R}$ & $-0.39^{* *}$ & 0.19 & -0.10 & -0.17 & 0.12 & & & & & & \\
\hline EI & $-0.46^{* *}$ & 0.16 & $-0.29^{*}$ & $-0.33^{*}$ & 0.10 & $0.60^{* *}$ & & & & & \\
\hline Dep & $-0.47^{* *}$ & $0.33^{*}$ & 0.03 & -0.06 & -0.13 & 0.20 & $0.31^{*}$ & & & & \\
\hline Anx & $0.30^{*}$ & $-0.26^{*}$ & $0.27^{*}$ & $0.28^{*}$ & 0.014 & -0.110 & $-0.24^{*}$ & $-0.29^{*}$ & & & \\
\hline Close & $-0.35^{* *}$ & $0.24^{*}$ & -0.066 & -0.024 & -0.172 & $0.32^{*}$ & $0.47^{* *}$ & $0.49^{* *}$ & -0.190 & & \\
\hline Age & -0.02 & -0.21 & $-0.30^{*}$ & -0.05 & 0.05 & -0.13 & -0.02 & 0.03 & -0.18 & 0.05 & \\
\hline
\end{tabular}

Notes. $\mathrm{PN}=$ past-negative time perspective; $\mathrm{PP}=$ past-positive time perspective; $\mathrm{PH}=$ present-hedonistic time perspective; $\mathrm{PF}=$ present-fatalistic time perspective; $\mathrm{F}$ = future time perspective; $\mathrm{R}=$ resilience; $\mathrm{EI}=$ emotional intelligence; Dep = dependent attachment style; Anx = anxiety attachment style; Close = close attachment style. $N=85$ for PN, PP, PH, PF, F, R, and Close. $N=$ 84 for EI, Dep, and Anx. All time perspective scores were measured by the ZTPI subscales. The resilience score was measured by the RS. The emotional intelligence scores were measured by the final PMI score. All attachment scores were measured by the RAAS subscales. ${ }^{*} p<0.05,{ }^{* *} p<0.001$.

The only correlation between age and the survey scales/subscales was moderate and found with time perspective subscale of present-hedonistic in the negative direction $r(84)=-0.30, p=0.006$. Further tests of colinearity were performed and the Tolerance and Variance Inflation Factors indicated that there was no significant amount of colinearity present.

Analysis of research questions. Each of the five ZTPI subscales was dichotomized into above and below the median and then each of the dichotomized subscales was used as a criterion variable in a logistic regression model. Recoding to above/below the median and doing a logistic regression was necessary because the data are not normally distributed and because the assumption of homoscedasticity was violated. The logistic regression was performed as an alternative type of analysis since the linear regression did not satisfy all assumptions. Logistic regression has fewer distributional assumptions than linear regression and is the analysis of choice when compared to multiple linear regression when the sample size is less than 100.

Stepwise logistic regression analysis was used to determine if any of the subject characteristics, gender, program phase, race, marital status, and income, and the resilience scale, PMI final, RAAS dependent, RAAS anxiety, and RAAS close were associated with the ZTPI subscales recoded into above or below the median. 
The SPSS program automatically removed the variables that were least associated using chi square tests. The criterion variables chosen by backward and forward stepwise regression were entered into a final model. This avoided having too many variables entered into the final model given that the total sample had less than 100 subjects.

Logistic regression assumes linearity of predictor variables with log odds so Box-Tidwell tests were performed to test for linearity. The Box-Tidwell tests were passed for all of the logistic regression independent variables. The product of the predictor variables with their natural $\log$ were entered into the model. A non-significant result implies that the relationship with the predictor variable is linear.

The following research questions were used to examine the strength of prediction of attachment style, resilience, and emotional intelligence on time perception. The questions were also used to examine how attachment style, resilience, and emotional intelligence interact to predict time perception in doctoral distant learners in the coursework and dissertation phase of their programs. Corresponding null hypotheses and alternative hypotheses are restated below.

Research question Q1: The first research question and its null and alternative hypotheses are restated below.

Q1: Does resilience predict time perspective in coursework and dissertation phases of a doctoral distance learning program?

$\mathrm{H1}_{0}$ : Resilience will not significantly predict time perspective during the coursework and dissertation phases of a doctoral distance learning program.

H1a: Resilience will significantly predict time perspective during the coursework and dissertation phases of a doctoral distance learning program.

Levels of psychological resilience were computed from the means of the overall scores on the RA. The RA is based on a self-report 7-point Likert scale, which addressed respondents' psychological resiliency ranging from 1 ("Agree") to 7 ("Disagree") producing ordinal data. A logistic regression was performed to determine the extent to which, if any, reliance predicted the five different types of time perspective: past-positive, past-negative, present-fatalistic, present-hedonistic, and future. The results of the logistic regression (see Table 3) showed that the resilience scale was one of the factors associated with having a score above the median on the future subscale $(p=0.005)$. The odds of a subject having a future subscale score above the median is increased by a factor of 1.115 for each unit increase in the resilience scale. Therefore, the null hypothesis was rejected for research question Q1 with respect to future time perspective orientation (i.e., psychological resilience did significantly predict a future time orientation in doctoral distance learning students. Resilience did not predict any other time perspective: past-positive, past-negative, present-fatalistic, or present-hedonistic, so for these latter time perspectives, the null hypothesis was not rejected.

Group statistics resulting from the performance of $t$-tests showed no significant difference, $p=0.43$, between the resilience scores of the students in the course work phase of their doctoral programs and the resilience scores of the students in the dissertation phase of their doctoral programs. However, there was a significant contribution from program phase to the overall prediction model for the future time perspective with regards to resilience. The manner in which reliance and program phase worked together to significantly predict future orientation is described under Q4.

Research question Q2: The second research question is restated below along with its associated null and alternative hypotheses. 
Q2: Does attachment style predict time perspective in coursework and dissertation phases of a doctoral distance learning program?

$\mathrm{H} 2_{0}$ : Attachment style will not significantly predict time perspective during the coursework and dissertation phases of a doctoral distance learning program.

H2a: Attachment style will significantly predict time perspective during the coursework and dissertation phases of a doctoral distance learning program.

Table 3

Prediction Model for Future Time Perspective Using Above Versus Below the Median Step-wise Logistic Regression

\begin{tabular}{|c|c|c|c|c|c|c|c|c|}
\hline & \multicolumn{5}{|c|}{ Variables in the equation } & \multicolumn{3}{|c|}{$95 \% \mathrm{CI}$} \\
\hline & $B$ & S.E & Wald & $d f$ & Sig. & $\operatorname{Exp}(B)$ & Lower & Upper \\
\hline $\mathrm{R}$ & 0.11 & 0.04 & 7.77 & 1 & 0.01 & 1.12 & 1.03 & 1.20 \\
\hline Dep & -0.93 & 0.35 & 7.10 & 1 & 0.01 & 0.39 & 0.20 & 0.78 \\
\hline MS & & & 7.78 & 2 & 0.02 & & & \\
\hline MS1 & 1.63 & 0.70 & 5.38 & 1 & 0.02 & 5.10 & 1.29 & 20.22 \\
\hline MS2 & -0.17 & 0.84 & 0.04 & 1 & 0.84 & 0.84 & 0.16 & 4.35 \\
\hline ProgP1 & 1.66 & 0.61 & 7.47 & 1 & 0.01 & 5.26 & 1.60 & 17.30 \\
\hline Gender1 & -1.77 & 0.69 & 6.66 & 1 & 0.01 & 0.17 & 0.05 & 0.65 \\
\hline Constant & -7.10 & 3.06 & 5.39 & 1 & 0.02 & 0.01 & & \\
\hline
\end{tabular}

Notes. $\mathrm{R}$ = resilience; Dep = dependent attachment style; MS = marital status; MS1 = marital status 1 (married); MS2 = marital status 2 (divorced); ProgP1 = program phase 1 (dissertation); Gender1 = female; and CI = confidence interval. The time perspective scores were measured by the future ZTPI subscale. Resilience scores were measured by the overall RA score. Attachment style was measured by the dependent attachment subscale of the RAAS. Marital status, ProgP1, and Gender1 were determined by the demographic questionnaire.

The RAAS (Collins \& Read, 1990; Appendix D) was used to measure participants' attachment style quantitatively using an 18-item, self-report, 5-point Likert scale. When completing the RAAS, participants in this study were asked to read each of the 18 statements and circle a number ranging from 1 ("Not at all Characteristic") to 5 ("Very Characteristic") that best indicated their feelings about each item. The RAAS has three subscales: Close, Anxious, and Dependent. The Close subscale measures the degree to which participants feel comfortable with closeness in relationships. The Depend subscale measures the degree to which participants believe they can depend on others. The Anxiety subscale measured fear of abandonment. The RAAS subscales correspond to the attachment styles of Secure, Avoidant, Anxious-Ambivalent (Anxious-Secure and Anxious-Avoidant) as reported by Collins and Read (1990). There is no overall score for the RAAS, so each subscale mean was used in the logistic regression.

A logistic regression was performed to determine the extent to which, if any, the three different types of attachment style predicted the five different types of time perspective: past-positive, past-negative, present-fatalistic, present-hedonistic, and future. The results of the logistic regression (see Table 4) showed that the RAAS anxiety attachment style was one of the factors associated with having a score above the median on the past-negative subscale $(p=0.034)$. The odds of a subject having a past-negative subscale score above the median is increased by a factor of 2.331 for each unit increase in the RAAS anxiety scale. Therefore, the null hypothesis was rejected for research question Q2 with respect to past-negative time perspective; the attachment style of anxiety did significantly predict a past-negative time orientation in doctoral distance learning students. 
Table 4

Prediction Model for Past-negative Time Perspective Using Above Versus Below the Median Step-wise Logistic Regression

\begin{tabular}{llllllrll}
\hline & \multicolumn{9}{c}{ Variables in the equation } & \multicolumn{3}{c}{$95 \%$ CI } \\
\cline { 2 - 10 } & $B$ & $S . E$ & Wald & $d f$ & Sig. & $\operatorname{Exp}(B)$ & Lower & Upper \\
\hline EI & -0.78 & 0.29 & 7.18 & 1 & 0.01 & 0.46 & 0.26 & 0.81 \\
Anx & 0.85 & 0.40 & 4.49 & 1 & 0.03 & 2.33 & 1.07 & 5.10 \\
Constant & 6.57 & 3.16 & 4.33 & 1 & 0.04 & 711.40 & & \\
\hline
\end{tabular}

Notes. EI = emotional intelligence; Anx = anxiety attachment style; and CI = confidence interval. The time perspective scores were measured by the past-negative ZTPI subscale. The emotional intelligence scores were measured by the final PMI score. The attachment scores were measured by the RAAS anxiety subscale.

The results of the logistic regression (see Table 5) also showed that the RAAS anxiety attachment style was one of the factors associated with having a score above the median on the past-positive subscale ( $p=$ 0.019). The odds of a subject having a past-positive subscale score above the median is increased by a factor of 0.412 for each unit increase in the RAAS anxiety scale. Therefore, the null hypothesis was rejected for research question Q2 with respect to past-positive time perspective orientation; the attachment style of anxiety did significantly predict a past-positive time orientation in doctoral distance learning students.

Table 5

Prediction Model for Past-positive Time Perspective Using Above Versus Below the Median Step-wise Logistic Regression

\begin{tabular}{lclllllll}
\hline & \multicolumn{9}{c}{ Variables in the equation } & \multicolumn{3}{c}{$95 \%$ CI } \\
\cline { 2 - 9 } & $B$ & $S . E$ & Wald & $d f$ & Sig. & Exp $(B)$ & Lower & Upper \\
\hline Anx & -0.89 & 0.38 & 5.50 & 1 & 0.02 & 0.41 & 0.20 & 0.87 \\
Constant & 1.46 & 0.70 & 4.30 & 1 & 0.04 & 4.30 & & \\
\hline
\end{tabular}

Notes. Anx = anxiety attachment style and CI = confidence interval. The time perspective scores were measured by the past-positive ZTPI subscale. Attachment scores were measured by the RAAS anxiety subscale.

The RAAS anxiety subscale did predict past-negative and past-positive time perspectives as reported above. The RAAS anxiety subscale did not significantly predict any other time perspective subscale: present-hedonistic, present-fatalistic, or future; therefore, the null hypothesis was accepted for these variables.

The results of the logistic regression (see Table 3) showed that the RAAS dependent attachment style was one of the factors associated with having a score above the median on the future time perspective subscale $(p=$ 0.008). The odds of a subject having a future subscale score above the median is increased by a factor of 0.393 for each unit increase in the RAAS dependent scale. Therefore, the null hypothesis was rejected for research question Q2 with respect to future time perspective orientation; the attachment style of dependent did significantly predict a future time orientation in doctoral distance learning students.

The RAAS dependent subscale did predict future time perspectives as reported above. The RAAS dependent subscale did not significantly predict any other time perspective subscale: past-negative, past-positive, present-hedonistic, or present-fatalistic; therefore, the null hypothesis was accepted for these variables.

The RAAS close subscale did not significantly predict any of the time perspective subscale: past-negative, past-positive, present-hedonistic, present-fatalistic of future; therefore, the null hypothesis was accepted for all of the time perspectives. 
Group statistics resulting from the performance of $t$-tests showed no significant difference between the attachment style subscales (dependent, $p=0.64$; anxiety, $p=0.80$; close, $p=0.28$ ) of the students in the course work phase of their doctoral programs and the attachment style subscales of the students in the dissertation phase of their doctoral programs. However, there was a significant contribution from program phase to the overall prediction model for the future time perspective with regards to the attachment style of dependent. The manner in which a dependent attachment style and program phase worked together to significantly predict future orientation is described under Q4.

Research question Q3: The third research question is restated below along with its associated null and alternative hypotheses.

Q3: Does emotional intelligence predict time perspective in coursework and dissertation phases of a doctoral distance learning program?

$\mathrm{H}_{3}$ : Emotional intelligence will not significantly predict time perspective during the coursework and dissertation phases of a doctoral distance learning program.

H3a: Emotional intelligence will significantly predict time perspective during the coursework and dissertation phases of a doctoral distance learning program.

Levels of emotional intelligence were computed from the means across the 18 items on the PMI (Greenberger et al., 1974; Appendix C). When completing the PMI, participants in this study were asked to read each of the 18 statements and circle a number ranging from 1 ("Strongly Disagree") to 4 ("Strongly Agree") that best indicates their feelings about each. Three of the nine subscales on the PMI were used: Self-Reliance, Work Orientation, and Identity yielding one summary score labeled Individual Adequacy. Only the summary score was used in the logistic regression.

A logistic regression was performed to determine the extent to which, if any, emotional intelligence predicted the five different types of time perspective: past-positive, past-negative, present-fatalistic, present-hedonistic, and future. The results of the logistic regression (see Table 4) showed that the emotional intelligence scale was one of the factors associated with having a score above the median on the past-negative subscale $(p=0.007)$. The odds of a subject having a past-negative subscale score above the median is increased by a factor of 0.457 for each unit increase in the emotional intelligence scale. Therefore, the null hypothesis was rejected for research question Q3 with respect to past-negative time perspective orientation; emotional intelligence did significantly predict a past-negative orientation in doctoral distance learning students.

The results of the logistic regression (see Table 6) also showed that the emotional intelligence scale was one of the factors associated with having a score above the median on the present-hedonistic subscale $(p=$ 0.034). The odds of a subject having a present-hedonistic subscale score above the median is increased by a factor of 0.575 for each unit increase in the emotional intelligence scale. Therefore, the null hypothesis was rejected for research question Q3 with respect to present-hedonistic time perspective orientation; emotional intelligence did significantly predict a present-hedonistic orientation in doctoral distance learning students.

The results of the logistic regression (see Table 7) also showed that the emotional intelligence scale was one of the factors associated with having a score above the median on the present-fatalistic subscale $(p=0.015)$. The odds of a subject having a present-fatalistic subscale score above the median is increased by a factor of 0.531 for each unit increase in the emotional intelligence scale. Therefore, the null hypothesis was rejected for research question Q3 with respect to present-fatalistic time perspective orientation; emotional intelligence did significantly predict a present-fatalistic orientation in doctoral distance learning students. 
Table 6

Prediction Model for Present-hedonistic Time Perspective Using Above Versus Below the Median Step-wise Logistic Regression

\begin{tabular}{llllllrll}
\hline & \multicolumn{9}{c}{ Variables in the equation } & \multicolumn{3}{c}{$95 \%$ CI } \\
\cline { 2 - 9 } & $B$ & S.E & Wald & $d f$ & Sig. & $\operatorname{Exp}(B)$ & Lower & Upper \\
\hline EI & -0.55 & 0.26 & 4.51 & 1 & 0.03 & 0.58 & 0.35 & 0.96 \\
ProgP1 & 1.00 & 0.48 & 4.33 & 1 & 0.04 & 2.72 & 1.06 & 6.95 \\
Constant & 5.34 & 2.64 & 4.10 & 1 & 0.04 & 208.35 & & \\
\hline
\end{tabular}

Note. ProgP1 = program phase 1 (dissertation); EI = emotional intelligence; and CI = confidence interval. The time perspective scores were measured by the present-hedonistic ZTPI subscale. Emotional intelligence scores were measured by the overall PMI score. ProgP1 was determined by the demographic questionnaire.

Table 7

Prediction Model for Present-fatalistic Time Perspective Using above versus below the Median Step-wise Logistic Regression

\begin{tabular}{lllllllll}
\hline & \multicolumn{9}{c}{ Variables in the equation } & \multicolumn{3}{c}{ 95\% CI } \\
\cline { 2 - 9 } & $B$ & $S . E$ & Wald & $d f$ & Sig. & $\operatorname{Exp}(B)$ & Lower & Upper \\
\hline EI & -0.63 & 0.26 & 5.94 & 1 & 0.02 & 0.53 & 0.32 & 0.88 \\
Constant & 6.61 & 2.69 & 6.03 & 1 & 0.01 & 739.892 & & \\
\hline Notes. EI $=$ & emotional & intelligence & and CI $=$ confidence interval. & The time perspective & scores & were measured by the
\end{tabular}
present-fatalistic ZTPI subscale. Emotional intelligence scores were measured by the overall PMI score.

Emotional intelligence did predict present-hedonistic, present-fatalistic, and past-negative time perspectives as reported above. Emotional intelligence did not significantly predict any other time perspective subscale: past-positive, or future; therefore, the null hypothesis was accepted for these variables.

Group statistics resulting from the performance of $t$-tests showed no significant difference, $p=0.82$, between the mean emotional intelligence scores of the students in the course work phase of their doctoral programs and the mean emotional intelligence scores of the students in the dissertation phase of their doctoral programs. However, there was a significant contribution from program phase to the overall prediction model for the present-hedonistic time perspective with regards to emotional intelligence. The manner in which emotional intelligence and program phase worked together to significantly predict present-hedonistic orientation is described under Q4 below.

Research question Q4: The fourth research question is restated below along with its associated null and alternative hypotheses.

Q4: How will resilience, attachment style, and emotional intelligence interact to predict time perspective during the coursework and dissertation phases of a doctoral distance learning program?

$\mathrm{H}_{0}$ : Resilience, attachment style, and emotional intelligence will not significantly interact to predict time perspective during the coursework and dissertation phases of a doctoral distance learning program.

H4a: Resilience, attachment style, and emotional intelligence will significantly interact to predict time perspective during the coursework and dissertation phases of a doctoral distance learning program. The results of each research question are stated below.

A step-wise logistic regression was performed to determine how resilience, attachment style, and emotional intelligence interact to predict time perspective during the coursework and dissertation phases of a doctoral distance learning program. Measures for each of the predictor variables have already been summarized 
in research questions Q1-Q3 above so only the results are presented here. The predictions are reported according to the model that best predicted each time perspective: past-negative, past-positive, present-hedonistic, present-fatalistic, and future and can be found in Tables 3-7 and corresponding Classification Tables in Tables 8-12.

Past-negative time perspective prediction model. The factors associated with having a score above the median on the past-negative scale were: RAAS anxiety scale $(p=0.034)$ and PMI final $(p=0.007)$ (see Table 4). The classification table (see Table 8 ) indicates that the model predicted the correct past-negative group for $69.0 \%$ of the subjects. $70.7 \%$ of the subjects below the median on the past-negative scale were correctly predicted, while $67.4 \%$ of the subjects who were above the median were correctly predicted. The odds of a subject having a past-negative score above the median is increased by a factor of 2.331 for each unit increase in the RAAS anxiety scale (see Table 4). The odds of a subject having a past-negative score above the median is decreased by a factor of 0.457 for each unit increase in the PMI final scale (see Table 4).

Table 8

Logistic Regression Analysis: Above vs. Below the Median Prediction Classification Table for Past-negative Time Perspective

\begin{tabular}{lcccc}
\hline & & \multicolumn{3}{c}{ Predicted } \\
\cline { 3 - 5 } & & & Past-negative & Percentage (\%) \\
\hline Observed & & 0.00 & 1.00 & Correct \\
Past-negative & 0.00 & 29 & 12 & 70.7 \\
& 1.00 & 14 & 29 & 67.4 \\
Overall \% & & & 69.0 \\
\hline
\end{tabular}

Past-positive time perspective prediction model. The factor associated with having a score above the median on the past-positive scale was: RAAS anxiety scale $(p=0.019)$ (see Table 5 ). The classification table (see Table 9) indicates that the model predicted the correct past-positive group for $67.9 \%$ of the subjects. $73.3 \%$ of the subjects below the median on the past-positive scale were correctly predicted, while $61.5 \%$ of the subjects who were above the median were correctly predicted. The odds of a subject having a past-positive score above the median is decreased by a factor of 0.412 for each unit increase in the RAAS anxiety scale (see Table 5).

Table 9

Logistic Regression Analysis: Above vs. Below the Median Prediction Classification Table for Past-positive Time Perspective

\begin{tabular}{lcccc}
\hline & & \multicolumn{3}{c}{ Predicted } \\
\cline { 3 - 5 } & & & Past-positive & Percentage (\%) \\
\hline Observed & & 0.00 & 1.00 & Correct \\
Past-positive & 0.00 & 33 & 12 & 73.3 \\
& 1.00 & 15 & 24 & 61.5 \\
Overall \% & & & 67.9 \\
\hline
\end{tabular}

Present-hedonistic time perspective prediction model. The factors associated with having a score above the median on the present-hedonistic scale were: PMI Final scale $(p=0.034)$ and Program phase $(p=0.037)$ (see Table 6). The classification table (see Table 10) indicates that the model predicted the correct present-hedonistic group for $67.9 \%$ of the subjects. $57.9 \%$ of the subjects below the median on the present-hedonistic scale were correctly predicted, while $76.1 \%$ of the subjects who were above the median were 
correctly predicted. The odds of a subject having a present-hedonistic score above the median is decreased by a factor of 0.575 for each unit increase in the PMI final scale (see Table 6). The odds of a subject having a present-hedonistic score above the median is increased by a factor of 2.715 or are 2.715 times greater for those in the dissertation phase as opposed to those in the course phase (see Table 6).

Table 10

Logistic Regression Analysis: Above vs. Below the Median Prediction Classification Table for Present-hedonistic Time Perspective

\begin{tabular}{lcccc}
\hline & \multicolumn{3}{c}{ Predicted } \\
\cline { 3 - 5 } & & & Present-hedonistic & Percentage (\%) \\
\hline Observed & 0.00 & 1.00 & Correct \\
PH & 0.00 & 22 & 16 & 57.9 \\
& 1.00 & 11 & 35 & 76.1 \\
Overall \% & & & 67.9 \\
\hline
\end{tabular}

Note. $\mathrm{PH}=$ present-hedonistic time perspective as measured by the ZTPI.

Present-fatalistic time perspective prediction model. The factor associated with having a score above the median on the present-fatalistic scale was PMI Final scale $(p=0.015)$ (see Table 7). The classification table (see Table 11) indicates that the model predicted the correct present-fatalistic group for $58.3 \%$ of the subjects. $57.5 \%$ of the subjects below the median of the present-fatalistic scale were correctly predicted, while $59.1 \%$ of the subjects who were above the median were correctly predicted. The odds of a subject having a present-fatalistic score above the median is decreased by a factor of 0.531 for each unit increase in the PMI final scale (see Table 7).

Table 11

Logistic Regression Analysis: Above vs. Below the Median Prediction Classification Table for Present-fatalistic Time Perspective

\begin{tabular}{lcccl}
\hline & & \multicolumn{3}{c}{ Predicted } \\
\cline { 3 - 5 } & & & Present-fatalistic & Percentage (\%) \\
\hline Observed & & 0.00 & 1.00 & Correct \\
PF & 0.00 & 23 & 17 & 57.5 \\
& 1.00 & 18 & 26 & 59.1 \\
Overall \% & & & 58.3 \\
\hline
\end{tabular}

Note. $\mathrm{PF}=$ present-fatalistic time perspective as measured by the ZTPI.

Future time perspective prediction model. The factors associated with having a score above the median on the future subscale were: Resilience scale $(p=0.005)$, Program phase $(p=0.006)$, RAAS dependent ( $p=$ $0.008)$, marital status $(p=0.020)$, and gender $(p=0.010)$ (see Table 3 ). The classification table (see Table 12) indicates that the model predicted the correct future subscale group for $70.4 \%$ of the subjects. $63.9 \%$ of the subjects below the median of the future subscale were correctly predicted, while $75.6 \%$ of the subjects who were above the median were correctly predicted. The following odds ratios for the future time perspective prediction model are reported in Table 3. The odds of a subject having a future subscale score above the median is increased by a factor of 1.115 for each unit increase in the Resilience scale. The odds of a subject having a future subscale score above the median is decreased by a factor of 0.393 for each unit increase in the RAAS dependent scale. The odds of a subject having a future subscore above the median is increased by a factor of 
5.101 or are 5.101 times greater for those who are married as opposed to those who are single. The odds of a subject having a future subscore above the median is decreased by a factor of 0.840 or are 0.840 times less for those who are divorced as opposed to those who are single. The odds of a subject having a future subscore above the median is decreased by a factor of 0.171 or are 0.171 times decreased for those who are female as opposed to those who are male. The odds of a subject having a future subscore above the median is increased by a factor of 5.259 or are 5.259 times greater for those in the dissertation phase as opposed to those in the course phase.

Table 12

Logistic Regression Analysis: Above vs. Below the Median Prediction Classification Table for Future Time Perspective

\begin{tabular}{lcccc}
\hline & & \multicolumn{3}{c}{ Predicted } \\
\cline { 3 - 5 } & & & Future & Percentage (\%) \\
\hline Observed & 0.00 & 1.00 & Correct \\
Future & 0.00 & 23 & 13 & 63.9 \\
& 1.00 & 11 & 34 & 75.6 \\
Overall \% & & & 70.4 \\
\hline
\end{tabular}

Assumptions and violations. There are four major assumptions in a multiple linear regression: reliability of measurement instruments, linearity, homoscedasticity, and normality. Cronbach's alpha was used to measure the internal consistency or reliability of the items comprising the survey subscales. If an assessment instrument is not reliable, an over-estimation can occur in the effect sizes of the other variables. Results showed that each measure had adequate or marginally adequate reliability. Even though two of the subscales of the PMI measuring emotional intelligence did not meet this assumption, the overall score of the PMI had adequate reliability. Since the overall score and not the subscores of the PMI were used in the prediction model, this assumption was not violated.

Multi-co-linearity means that variables have a linear relationship or are nearly perfect linear combinations of each other. Multiple linear regression analysis requires a linear relationship between each predictor variable and the criterion variable. If there is not, a Type II error and under-estimation may occur. A Type I error may also occur as an over-estimation for the other criterion variable if it shares variance will the first criterion variable. Multiple linear regression analysis also requires that there be no significant linearity amongst predictor variables. Co-linearity between variables was examined using co-linearity tests. All tolerance and VIF values where within acceptable ranges, indicating that there was no linearity violation for multiple linear regression or logistic regression analyses.

The third requirement is that homoscedasticity exists, meaning that variance of errors is the same for all levels of the predictor variables. If not, heteroscedasticity can result in Type I error. Levene's tests of homogeneity and robust tests of equality of variance, Welch and Brown-Forsythe, were used to test for homoscedasticity. Levene tests are used when the sample size is small and when groups have difference sample sizes, both of which were true for this study. The Levene test result was significant (meaning that the variances are not the same so there was a violation of this assumption) in this study, so the "non-equal variance" $t$-test result was used.

Normality is a fourth requirement of multiple linear regression. Analysis of variance was used to compare the averages of the scales and subscales where the grouping variable has more than two levels. A significant 
ANOVA result determines that the means are not all the same. Two significant ANOVA results were found: race and marital status. The results of multiple comparisons tests (Least Significant Difference test (LSD) and Tamhane) showed that the means on the past-positive scale were significantly different for the different groups of the demographic variable of race; the mean of the white group was significantly lower than the other/unknown group. The white group did not pass the normality test, which is an assumption failure of the ANOVA because significance means that the distribution is significantly different from a normal distribution. A non-parametric median test to compare the medians across the race groups was done as a substitute for the ANOVA because median tests do not have a distribution assumption. The result of the median test was not significant; hence, the results of the ANOVA were not used and the assumption of normality was violated for multiple linear regression analysis.

The means on the future subscale were significantly different for the different groups of the demographic variable of marital status. The LSD multiple comparisons test indicated that the mean of the single group was significantly lower than the married group. The single group did not pass the normality test, which is an assumption failure of the ANOVA because significance means that the distribution is significantly different from a normal distribution. A non-parametric median test to compare the medians across the marital status groups was done as a substitute for the ANOVA because median tests do not have a distribution assumption. The result of the median test was not significant; hence, the results of the ANOVA were not used and the assumption of normality was violated a second time for multiple linear regression analysis.

The results reported above indicate that in this study, two of the four assumptions were met: those for reliability and linearity. Two of the assumptions were not met: those for homoscedasticity and normality. Since two of the assumptions were violated, multiple linear regression analysis could not be used and an alternative method of analysis was required.

Many of the assumptions necessitated by multiple linear regression analysis are not needed in order to perform a logistic regression. There need not be a linear relationship between criterion and predictor variables because a non-linear log-transformation is used to predict odds ratios in a logistic regression analysis. Residuals or error terms and predictor variables do not need to be multivariate normal in a logistical regression. Predictor variables do not need to be metric (ratio or interval scaled). Homoscedasticity is not necessary in a logistical regression.

Yet, there are six assumptions that do need to be met in order to have a robust logistic regression analysis. The first assumption is that a binary logistic regression (the type of logistic regression that was performed in this study) requires that the criterion variable be binary. Therefore, all of the criterion subscales were dichotomized into above and below the median and then each of the dichotomized subscales was used as a criterion variable in a logistic regression model. However, using dichotomous levels may result in a loss of information.

A second assumption is that the desired outcome must be the factor level 1 of the criterion variable as opposed to 0 because a binary logistic regression assumes that $\mathrm{P}=(\mathrm{Y}=1)$, the probability of the event occurring. In this study, the subscales of the ZTPI were re-coded in a manner to meet this requirement so that scores above the mean indicated a time perspective bias characteristic of the subscale category (i.e., subjects who scored above the mean on the ZTPI future time perspective subscale were deemed to have a future time perspective orientation).

A third assumption of a logistic regression is that the model should have a correct fit. The model should not be over or under fit. A sureness of fit means that all meaningful variables should be used in the model, but 
only variables that are meaningful. In order to meet this assumption, a step-wise logistic regression was employed.

A fourth assumption is that there will be a large enough sample size: at least 10 participants for each predictor variable. The regression model composed of the most variables was the model that predicted a future time perspective. The number of variables entered to predict the future orientation was six, so at least 60 participants were necessary for this regression. All other models had fewer than six variables. In this study, there were a total of at least 82 participants, so the sample size assumption was met.

Logistic regression also assumes that the predictor variables will have a linear relationship with log odds or the strength of the prediction may be underestimated and the analysis may too easily reject relationships. This assumption was met as per Box-Tidwell tests.

The final assumption of a logistic regression is that error terms must be independent. There can be no before and after analyses and no matching pairs. Instead, each analysis must be independent. Multiple co-linearity should be minimal so that each predictor variable should be independent from each other. Since there were some significant correlations between certain predictor variables as mentioned in the section above entitled Correlations, more rigorous tests were used to determine if co-linearity existed. The Tolerance and Variance Inflation Factors generated as a result of these tests showed that co-linearity was not significantly present in this study to warrant a violation of assumption. Results of all tests of assumptions for logistic regression analysis showed that there were no assumption violations.

\section{Evaluation of Findings}

The meaning of the results of this study is discussed below. The results are interpreted in light of Zimbardo and Boyd's $(1999 ; 2008)$ theory of time perspective and conceptual frameworks described in Chapters 1 and 2. The results are compared with findings in the literature and potential explanations for unexpected or conflicting results are given. This section is organized by evaluating the findings of the predictor variables: resilience (research Q1), attachment (research Q2), emotional intelligence (research Q3), and program phase (Q1-Q3,) followed by results from the demographic variables of marital status and gender, and finally, an evaluation of the overall time perspective prediction models (Q4).

Resilience and time perspective. Resilience predicted one time perspective, the future orientation.

Resilience predicted future (+). Resilience predicted a future time perspective in the positive direction. High scores on resilience predicted a future time perspective. Specifically, resilience was one of the factors associated with having a score above the median on the future. The odds of a subject having a future subscale score above the median was increased by a factor of 1.115 for each unit increase in the resilience scale. Therefore, the null hypothesis was rejected for research question Q1 with respect to future time perspective orientation (i.e., psychological resilience did significantly predict a future time orientation in doctoral distance learning students). Resilience did not predict any other time perspective: past-positive, past-negative, present-fatalistic, or present-hedonistic, so for these latter time perspectives, the null hypothesis was not rejected.

Resilience is the process by which individuals interact and cope with their environment and includes factors that promote well-being (Zautra et al., 2010). This ability to cope despite barriers allows for development and achievement of long-term goals, which is characteristic of a future orientation. Individuals scoring high on future orientation tended to also have high ego functioning, high self-esteem, and were able to respond to perceived threats in their environment without becoming overly anxious (Zimbardo \& Boyd, 1999). 
These characteristics are also part of psychological resilience. In students, future orientation was positively correlated with grade point average, hours of studying per week, and creativity (Zimbardo \& Boyd, 1999). Resilience may be necessary for students to deal with problems as they arise in order to continue to study and achieve good academic outcomes.

Attachment style and time perspective. Anxiety attachment style predicted past-negative (+). An anxious attachment style predicted the time perspective of past-negative. The prediction was in the positive direction so that high scores on the anxious attachment subscale predicted a time perspective of past-negative. Specifically, the RAAS anxiety attachment style was one of the factors associated with having a score above the median on the past-negative subscale. The odds of a subject having a past-negative subscale score above the median was increased by a factor of 2.331 for each unit increase in the RAAS anxiety scale. Therefore, the null hypothesis was rejected for research question Q2 with respect to past-negative time perspective; the attachment style of anxiety did significantly predict a past-negative time orientation in doctoral distance learning students.

This finding is supported by the literature in that trait anxiety showed a strong positive correlation with past-negative time perspective and a negative correlation with friendliness (Zimbardo \& Boyd, 1999). Individuals who have experienced past negative events, which they find difficult to decathect, may have developed an anxiety attachment style as a result of these negative or traumatic events. Anxiety attachment style in adults is usually characterized by difficulty with the ability to trust people, fear of abandonment and disappointment, difficulty with the ability to be alone, fear of the loss of a love object, and fear of death and annihilation (Bodie et al., 2011; Bowlby, 1979; Thompson, 2008). Zimbardo and Boyd (1999) found that individuals with past-negative time perspectives had unsatisfactory and minimal interpersonal relationships and reported having fewer close friendships as compared to those with other time perspectives. Focusing on negative past events/memories in the present indicates that individuals with this time perspective may have unresolved issues regarding memories of their past, which may be causing them to be anxious in current relationships.

Anxiety attachment style predicted past-positive (-). An anxiety attachment style predicted the time perspective of past-positive. The prediction was in the negative direction so that low scores on the attachment subscale of anxiety predicted a time perspective of past-positive. Specifically, the RAAS anxiety attachment style was one of the factors associated with having a score above the median on the past-positive subscale. The odds of a subject having a past-positive subscale score above the median was increased by a factor of 0.412 for each unit increase in the RAAS anxiety scale. Therefore, the null hypothesis was rejected for research question Q2 with respect to past-positive time perspective orientation; the attachment style of anxiety did significantly predict a past-positive time orientation in doctoral distance learning students.

This finding is supported by the literature in that trait anxiety showed a strong negative correlation with past-positive time perspective and a strong positive correlation with friendliness (Zimbardo \& Boyd, 1999). If an anxiety attachment style predicted a past-negative time perspective in the positive direction, it is not unusual to find that low scores on the attachment subscale of anxiety predicted a past-positive time perspective. Individuals who have the ability to trust others in close relationships and bond with others without excessive anxiety or enmeshment may have developed these abilities through past positive experiences with their primary caretaker. If a mother of an infant is calm and able to consistently soothe her baby when the infant is in distress, the baby has a better chance of developing his or her ability to manage anxiety as development continues into 
adulthood. The ability to soothe or be soothed during infancy may lead to a positive view and/or memories of the past.

Dependent attachment style predicted future (-). A dependent attachment style predicted a future time perspective. The prediction was in the negative direction so that low scores on the dependent attachment subscale predicted a time perspective of future orientation. Specifically, the RAAS dependent attachment style was one of the factors associated with having a score above the median on the future time perspective subscale. The odds of a subject having a future subscale score above the median is increased by a factor of 0.393 for each unit increase in the RAAS dependent scale. Therefore, the null hypothesis was rejected for research question Q2 with respect to future time perspective orientation; the attachment style of dependent did significantly predict a future time orientation in doctoral distance learning students.

Low scores on the dependent attachment subscale may indicate the ability to form relationships that are not overly enmeshing. When two individuals come together in a relationship but are able to maintain their emotional independence, stability, and self-integrity, the relationship tends to allow those individuals a chance to grow in the relationship and continue to grow as individuals. These types of relationships are usually more successful with respect to individuation than those based on dependency and need. The sharing of life experiences between two people in this healthy manner can support and enhance each individual's ability to feel comfortable in the relationship, which may lead to less impulsivity and the ability to set long-term goals for the future, and hence be successful in life.

Emotional intelligence and time perspective. Emotional intelligence predicted past-negative (+). Emotional intelligence predicted past-negative time perspective in the positive direction. High scores in emotional intelligence predicted a past-negative time perspective. Specifically, the emotional intelligence scale was one of the factors associated with having a score above the median on the past-negative subscale. The odds of a subject having a past-negative subscale score above the median was increased by a factor of 0.457 for each unit increase in the emotional intelligence scale. Therefore, the null hypothesis was rejected for research question Q3 with respect to past-negative time perspective orientation; emotional intelligence did significantly predict a past-negative orientation in doctoral distance learning students.

This finding is supported by the literature in that aggression and depression both showed a strong positive correlation with past-negative time perspective. Emotional stability and impulse control were both negatively correlation with past-negative time perspective (Zimbardo \& Boyd, 1999). Zimbardo and Boyd found a positive correlation between ego control and past-negative time perspective. Individuals who have experienced events in their childhood that were negative may have had ample opportunity to learn to develop emotional intelligence through these experiences if they were not too overwhelming or if they had help dealing with them. The ability to recognize and manage emotions in self and others takes practice. It could be that students with negative past experiences sought therapy to develop appropriate methods to manage their feelings. It could also be that students may currently be in therapy to deal with negative past experiences so are focused in the past and on negative experiences.

Emotional intelligence predicted present-hedonistic (-). Emotional intelligence predicted present-hedonistic time perspective in the negative direction. Low scores on emotional intelligence predicted a present-hedonistic time perspective. Specifically, the emotional intelligence scale was one of the factors associated with having a score above the median on the present-hedonistic subscale $(p=0.034)$. The odds of a subject having a present-hedonistic subscale score above the median is increased by a factor of 0.575 for each unit increase in 
the emotional intelligence scale. Therefore, the null hypothesis was rejected for research question Q3 with respect to present-hedonistic time perspective orientation; emotional intelligence did significantly predict a present-hedonistic orientation in doctoral distance learning students.

This result is supported by the literature, which reported that present-hedonistic time perspective showed positive correlations with aggression, depression, and ego control and negative correlations with emotional stability and impulse control (Zimbardo \& Boyd, 1999).

Low scores on emotional intelligence indicate an inability to manage one's emotions and the inability to manage one's emotions often leads to impulsive behavior, which is characteristic of individuals with a present-hedonistic time perspective. Individuals who cannot stave off impulses usually act on them in order to gain pleasure or avoid negative feelings instead of being able to tolerate or refocus their affects. Present-hedonistic time perspective comprises individuals who tend to be impulsive and focused on satisfying impulses as they arise in their daily lives.

Emotional intelligence predicted present-fatalistic (-). Emotional intelligence predicted a present-fatalistic time perspective in the negative direction. Low scores on emotional intelligence predicted a present-fatalistic time perspective. Specifically, the emotional intelligence scale was one of the factors associated with having a score above the median on the present-fatalistic subscale $(p=0.015)$. The odds of a subject having a present-fatalistic subscale score above the median is increased by a factor of 0.531 for each unit increase in the emotional intelligence scale. Therefore, the null hypothesis was rejected for research question Q3 with respect to present-fatalistic time perspective orientation; emotional intelligence did significantly predict a present-fatalistic orientation in doctoral distance learning students.

This result is supported by the literature, which reported that present-fatalistic time perspective showed positive correlations with aggression, depression, and ego control and negative correlations with emotional stability and impulse control (Zimbardo \& Boyd, 1999).

Individuals who score low on emotional intelligence tend to lack the ability to recognize and respond to emotional states of others as well as their own. They are unable to use emotional awareness to problem solves and engage in relationships (Côté et al., 2011), which may cause them to experience the present as fatalistic. Individuals with a present-fatalistic time perspective tend to believe that they cannot influence their futures because they are helpless to do so. This helplessness may come from an inability to problem solve and gain help from others. Instead, the future will be determined by forces outside themselves or fate, which they cannot control. Individuals scoring high on the present-fatalistic subscale have been reported to be more depressed, anxious, and aggressive than individuals with other time perspectives (Zimbardo \& Boyd, 1999).

Program phase. Dissertation phase predicted present-hedonistic (+). Dissertation phase predicted a present-hedonistic time perspective. Participants in the dissertation phase of their online distance learning programs tended to score above the mean on the present-hedonistic subscale of the time perspective inventory. Specifically, the odds of a subject having a present-hedonistic score above the median was increased by a factor of 2.715 or were 2.715 times greater for those in the dissertation phase as opposed to those in the course phase.

Individuals with a present-hedonistic time perspective tend to be oriented toward present excitement, pleasure, and enjoyment (Zimbardo \& Boyd, 1999). Zimbardo and Boyd (1999) emphasized the negative aspects of a present-hedonistic time perspective with respect to impulsivity, living for the moment, and putting duties off in order to engage in pleasure seeking activities such as drinking and using drugs. They did not 
describe the present-hedonistic time perspective in a positive manner. In this study, students in the dissertation phase of their online distance learning programs may have been oriented towards the present excitement of completing their dissertation. Working on their dissertation may have given them pleasure and they may have excluded many other activities in order to focus on the hedonistic task of completing their research.

Another explanation for this finding may be that under the structure of taking classes, student may feel more pressure to complete assignments and less freedom to satisfy themselves with day to day pleasures and/or to achieve a daily balance of work, school, and leisure necessary to decrease stress and remain productive and happy. Once in the dissertation phase, participants may have gained a sense of confidence in being able to complete the program and allowed themselves the freedom to relax and enjoy themselves.

Dissertation phase predicted future (+). Dissertation phase predicted a future time perspective in the positive direction. Participants in the dissertation phase of their online distance learning programs tended to score above the mean on the future subscale of the time perspective inventory. Specifically, the odds of a subject having a future subscore above the median was increased by a factor of 5.259 or were 5.259 times greater for those in the dissertation phase as opposed to those in the course phase.

There have not been any published studies to date that involved examining the time perspective of doctoral students, but studies using college students as subjects have reported a strong correlation with academic success and future orientation (Zimbardo \& Boyd, 1999). Future orientation allowed subjects to see ahead, delay gratification, set goals, and manage their time, whereas present oriented individuals had the highest college attrition rate (Boyd \& Zimbardo, 2005; de Volder \& Lens, 1982). Individuals who scored high in future orientation were more punctual for class, apt to complete homework before due dates, inclined to begin assignments on time, and skilled in time management than their classmates (Harber et al., 2003). Future oriented college students also tended to utilize successful learning strategies such as reproductive and deep-level cognitive processing more often than students with other time orientations (Horstmanshof \& Zimitat, 2007). In addition, persistence (Peetsma, 1994; Zaleski, 1987), level of engagement (Peetsma, 1994), satisfaction (Zaleski, 1987), number of hours spent in school work (Peetsma, 1994) and grade point average (Zimbardo \& Boyd, 1999) were related to future orientation among college students. Future orientation was also associated with academic achievement in high school learners (Anderman et al., 1999; de Volder \& Lense, 1982; Groth, 1973; Honora, 2002; Shell \& Husman, 2001).

The results from the literature above could support the finding of the current study that the dissertation phase of the doctoral program predicted a future time perspective, since in order to achieve the level of "all but dissertation", students may have had to be future oriented. It is possible that students with other orientations of time perspective dropped out of their doctoral programs before making it to the dissertation phase. Investigators (Boniwell \& Zimbardo, 2003, 2004; Holman \& Silver, 1998; Zimbardo \& Boyd, 2008) have reported that people who were successful in life (financially, socially, work-wise, and with the ability to form and maintain intimate relationships) scored moderately high in present-hedonistic and future orientations, so it is not surprising that subjects in the dissertation phase tended to score high on both present-hedonistic and future orientations.

Marital status and time perspective. Marriage predicted future (+). Marriage predicted future time orientation in the positive direction. Participants who were married tended to have a future time perspective. Specifically, the odds of a subject having a future subscore above the median was increased by a factor of 5.101 or were 5.101 times greater for those who were married as opposed to those who were single. The odds of a 
subject having a future subscore above the median was decreased by a factor of 0.840 or were 0.840 times less for those who are divorced as opposed to those who are single.

Having the support of a marital partner and the stability of being married (although not all marriages are supportive and stable) may enhance the ability to think about and set long-term goals that involve family members and work together to achieve them. This finding is supported by the literature in that future orientation correlated with successful long-term intimate relationships and overall success in life (Zimbardo \& Boyd, 1999).

Gender and time perspective. Being female predicted future (-). Being female predicted future time perspective in the negative direction. Participants who were female tended to score low in future time orientation. The odds of a subject having a future subscore above the median was decreased by a factor of 0.171 or were 0.171 times decreased for those who were female as opposed to those who were male.

This finding is supported by the literature, which has shown that adult female online learners more so than their male counterparts tended to put their school work second to other roles such as homemaker, mother, caretaker, when events in these roles necessitated immediate action (Zembylas, 2008). Ferrari et al. (2012) found that as female adolescents matured into older adolescents, their levels of future time perception decreased as a result of social pressure.

\section{Overall Model Predictions}

Past-negative. Past-negative time perspective was predicted by high scores in emotional intelligence and high scores for an anxiety attachment style. An anxiety attachment style has been characterized by the fear of abandonment (Collins \& Read, 1990). Since attachment styles are developed within the first year of life, a child with an anxiety attachment style has most likely experienced some form of early abandonment, which may have continued into childhood depending on the quality and consistency of bonding with his primary caretaker (Bowlby, 1979). The result may have been repressed or conscious painful negative past memories. In this fashion, the fear of abandonment may have developed early in childhood, resulting in an anxiety attachment style that lasted into adulthood. Children who fear abandonment may become experts at the skills of identifying, understanding, and predicting the emotions of others in relation to themselves in order to avoid more abandonment and its concomitant emotions. Emotional intelligence has been characterized by the ability to identify, understand, and predict emotions in one's self and others (Côté et al., 2011), so children with abandonment issues may also score high on emotional intelligence that was developed in part to avoid abandonment. A past-negative time perspective orientation has been characterized by the tendency to dwell on past-negative events (Zimbardo \& Boyd, 1999; 2008). Since the fear of abandonment is usually rooted in early past infant experiences and an attempt to manage it may have begun in childhood, the fear of abandonment and its management, for some individuals, may be focused on the past and its negative experiences. Hence, the fear of abandonment (as measured by an anxiety attachment style) may lead to the development of emotional intelligence necessary to deal with the fear rooted in the past resulting in individuals who tend to dwell on negative past experiences that caused them anxiety in order to avoid more anxiety in their current lives. This is one explanation for how emotional intelligence and anxiety attachment style may have worked together to predict a past-negative time perspective.

Past-positive. Past-positive time perspective was predicted by low scores for an anxiety attachment style. This finding is supported by the literature in that trait anxiety showed a strong negative correlation with 
past-positive time perspective and a strong positive correlation with friendliness (Zimbardo \& Boyd, 1999). The anxiety attachment scale measured fear of abandonment. A lack of abandonment fear may contribute to the development of a past-positive time perspective. Individuals who have not experienced traumatic abandonment early in life and who have established object constancy may recall their pasts as being positive.

Individuals who have the ability to trust others in close relationships and bond with others without excessive anxiety or enmeshment may have developed these abilities through past positive experiences with their primary caretaker. If a mother of an infant is calm and able to consistently soothe her baby when the infant is in distress, the baby has a better chance of developing his or her ability to manage anxiety as development continues into adulthood, according to Winnicott's theory of the "good-enough mother" (1953). The ability to soothe or be soothed during infancy may lead to a positive view and/or memories of the past.

Present-hedonistic. Present-hedonistic time perspective was predicted by low scores in emotional intelligence and the dissertation phase of the online distance doctoral program. Emotional intelligence and less structure may have worked together to predict a present-hedonistic time perspective in the following manner. Less structure in the dissertation phase may have allowed students with low emotional intelligence the opportunity to act in a hedonistic manner; perhaps because they could willfully enjoy themselves more in day-to-day pleasures because their goal of completing a dissertation was longer than the goal of completing a course or because the structure of the course work phase helped them be less hedonistic and more focused on future goals such as completing assignments. Without the help of structure, during the dissertation phase, students with low emotional intelligence scores may have fallen prey to engaging in more hedonistic behavior.

Present-fatalistic. Present-fatalistic time perspective was predicted by low scores in emotional intelligence. Since there was only one significant predictor of present-fatalistic time perspective (emotional intelligence), the evaluation of this model has already been addressed in research Q3 above but will be restated here. A present-fatalistic time perspective was characterized by the belief that the future is predetermined and cannot be changed by actions, thoughts, or beliefs (Zimbardo \& Boyd, 2008); hence, individuals who scored high on present-fatalistic orientation also tended to be depressed and anxious (Zimbardo \& Boyd, 1999). Present-fatalistic time perspective is also marked by an external locus of control and a resignation not only to the present but also to the future. Instead, according to Zimbardo and Boyd (1999), life is determined by fate in the minds of individuals scoring high in present-fatalistic time perspective. The construct of emotional intelligence includes an awareness of the emotions of one's self and others and the ability to understand and manage emotions in order to actively solve problems and direct actions (Côté et al., 2011). If individuals do not develop emotional awareness, they may also resign themselves to having to accept current situations because they cannot manage them. Individuals who cannot manage current situations may feel helpless and give up trying to take action, effect change, or exert control over themselves and their lives, thereby producing a present-fatalistic time perspective.

Future. Future time perspective was predicted by high scores in resilience, low scores in dependent attachment style, program phase (dissertation), gender (male), and marital status (married). From these results, it appears that future oriented students in this study tended to be married men who were resilient, independent, and in the dissertation phase of their online doctoral program. Individuals with dependent attachment styles believe they can depend on others to get their needs met (Collins \& Read, 1990). Conversely, individuals with low scores in dependent attachment style believe that they cannot depend on others to meet their needs. Individual who scored low on dependent attachment style who are also resilient may depend on themselves to 
meet their needs instead of others. In this way, resilience and attachment style may have worked together in married male participants in this study to predict a future time perspective, which has been characterized as one in which individuals tended to be organized, productive, hard-working, and academically successful despite barriers (Zimbardo \& Boyd, 1999; 2008).

\section{Summary}

Preliminary tests (Chronbach's alpha, $t$-tests, Levene tests, ANOVAs, Welch, Brown-Forsythe, LSD, Tamhane, Kolmogorov-Smirnova, and Shappiro-Wilk) revealed that there were two violations of multiple linear regression analysis: normality and homoscedasticity in this study. Therefore, results of step-wise logistic regression analyses were presented. Logistic regression analysis revealed that resilience, attachment style, and emotional intelligence each individually predicted time perspective in online distance learning doctoral students. The details of the types of predictions were reported above for each predictor and criterion variable. An explanation of the findings was offered for each prediction based on the literature and relevant theories. An overall model of how the predictor variables of resilience, attachment style, emotional intelligence, and the demographic variables of marital status and gender worked together to predict each time perspective category for online distance learning doctoral students in the course work phase and dissertation phase of their programs was described and explained in context of Zimbardo and Boyd's $(1999 ; 2008)$ theory of time perspective. 


\section{Chapter 5: Implications, Recommendations, and Conclusions}

The perception of time (the use of temporal categories to conceptualize experiences) affects human behavior (Zimbardo \& Boyd, 2008). The application of time perception to academia is of interest because distance learning programs are becoming prevalent; however, the attrition rate for all but dissertation distance learners has been reported to be as high as $80 \%$ (Flood, 2002). Students with future time perspective orientations tend to have better academic outcomes than those with past or present according to Zimbardo and Boyd's (1999; 2008) psychology of time model. Zimbardo and Boyd's (1999; 2008) linear unidirectional application of time perspective to academic achievement is limited because of the exclusion of certain variables that may influence the development and maintenance of time perspective and, hence, academic achievement. In addition, Zimbaro and Boyd's (1999; 2008) theory claims that time perception continually develops and changes but does not explain which aspects of structure and discipline affect time perspective or its plasticity. Given these gaps in their theory, resilience, emotional maturity, and attachment style were examined for their influence on time perception because of their stated relationships to behavior and time perception in the literature. Yet, the manner in which resilience, emotional maturity, and attachment style may predict time perception is unknown.

The purpose of this nonexperimental quantitative survey study was two-fold. First, it was used to identify what predictive role emotional intelligence, attachment style, and resilience (predictor variables) had on time perception (criterion variable) in doctoral distance learners. Second, the study was used to ascertain how emotional intelligence, attachment style, and resilience (predictor variables) interacted to predict time perception (criterion variable). Participants were 93 distance learning doctoral candidates from universities throughout the United States with an online format; 51 participants were in the coursework phase, 38 participants were in the dissertation phase of their program, and four did not indicate which phase of the program they were in. They were recruited from various universities and professional doctoral forums through the internet. Survey data were collected using Surveymonkey between October 2 and December 31, 2013. Questions regarding demographics, time perception, personal relationships, resilience, and emotional intelligence were asked. Data gathered online from the Zimbardo Time Perception Inventory, Resilience Scale, Psychosocial Maturity Inventory, Revised Adult Attachment Scale, and a demographic questionnaire were analyzed using step-wise logistic regression analyses.

Limitations of this study that may have affected its results include those having to do with the nature of the survey assessments, participants, and design. They are described in more detail further on in this chapter.

\section{Ethical Considerations}

This researcher took the following steps to ensure that this study complied with the ethical standards of protection from harm, informed consent, right to privacy, and honesty with professional colleagues. This researcher made every effort to protect all participants from harm during their participation in this study. An application to the Institutional Review Board of Northcentral University was made by this researcher and approval granted for this non-experimental study before any data was collected. The status granted was "exempt" for two reasons. First, the researcher of this study was not in any way biased toward inclusion of vulnerable population groups such as fetuses, prisoners, children, elders, pregnant woman, females, members of minority groups and their sub-populations, or individuals with disabilities. This researcher made sure that the 
surveys were equally available to any gender and racial/ethnic/religious group and did not discriminate against people with alternative sexual orientations. Second, this project did not involve invasive procedures or other medical or therapeutic interventions. This researcher did not misinform or mislead participants in any way. No risk to the subjects existed beyond those of everyday living. However, minimal risk to participants may have arisen from psychological reactions to certain questions on the survey (Borden \& Abbott, 2005). Participants were granted permission to stop the survey at any point or to skip a question if they were not comfortable answering it. A statement to this effect was included in the Consent Form posted on the first page of the Surveymonkey link (https://www.surveymonkey.com/s/ZHZS3ST) by this researcher. Such individuals had an option of using the researcher's e-mail address that was provided by this researcher on the Informed Consent Form in case concerns arose during the completion of the survey, but none did. In addition, a comment section was added to the end of the survey to provide an opportunity for participants to raise issues regarding the study. Participants who wanted more information about time perspectives could read Zimbardo and Boyd's book (2008) that was referenced in the survey. In this book, Zimbardo and Boyd (2008) explain the value of each time perspective so participants who had negative feelings about the survey or their results could gain a more positive understanding of their time perspective. Participants who experienced negative feelings after taking the survey could have contacted this researcher (who has a doctorate in psychoanalysis and is a licensed, certified psychoanalyst) at the contact information listed on the Invitation to Participate and Consent Forms and this researcher would have tried to help individuals feel better, but no one requested this service. Therefore, harm to subjects was assumed to be minimal.

All participants provided informed consent via the Consent Form, which included a brief introduction to the study: its purpose, nature, risks, benefits, and participants' status (voluntary). Participant's submission of a survey also indicated consent. The Invitation to Participate flyer (see Appendix F) and the Cover Letter introducing the study (see Appendix $\mathrm{H}$ ) were sent by this researcher to the chairpersons/directors and also included this information. There was no monetary compensation or incentives for the participants in this study. Therefore, the nature of this study and participants' involvement was clarified so informed consent could be given prior to completion of the survey.

The privacy of all participants was upheld by this researcher. Participants did not give the researcher their names. The surveys contained no questions that ask for identifying information, so no personal data was gathered or shared. The researcher did not know the identity of the participants beyond age, gender, educational level, school, marital status, economic status, ethnicity, and learning environment. Surveymonkey was password-protected for additional security. Surveys were sent from the participants to a Gmail account created by the researcher for storing responses safely, which was accessed by the researcher only and password protected. Each survey was coded by the researcher with an identification number for storing data. Participants may have elected to give their e-mail address to the researcher to receive their scores. Under this condition, only the researcher had access to these e-mail addresses. The researcher coded the e-mails, calculated the scores of each survey, and sent the scores to participants who requested them. When the study was finished, the Gmail account was terminated by the researcher so it could not be accessed afterwards. Results were reported in an aggregated format. All hard copies of data material will be destroyed by a paper shredder and all electronic files will be deleted from the researcher's computer 5 years post completion of the study. By using these methods, participants' anonymity was preserved throughout the duration of this study. 
Chapter 5 discusses the implications of the findings of this study and how potential limitations may have affected the interpretation of the results. Chapter 5 also describes how the results respond to the study's problem, fit with the purpose, demonstrate significance, and contribute to the existing literature described in Chapter 2. Recommendations for practical applications of the results of this study are made along with recommendations for future research. Key points in Chapter 5 are then summarized in the Conclusion section of this chapter.

\section{Implications}

The following research questions were used to examine the strength of prediction of attachment style, resilience, and emotional intelligence on time perception. The questions were also used to examine how attachment style, resilience, and emotional intelligence interact to predict time perception in doctoral distant learners in the coursework and dissertation phase of their programs. Corresponding null hypotheses and alternative hypotheses are restated below and their implications discussed.

Research question Q1: Does resilience predict time perspective in coursework and dissertation phases of a doctoral distance learning program?

$\mathrm{H} 1_{0}$ : Resilience will not significantly predict time perspective during the coursework and dissertation phases of a doctoral distance learning program.

H1a: Resilience will significantly predict time perspective during the coursework and dissertation phases of a doctoral distance learning program.

Resilience did predict time perspective in that high scores in resilience predicted a future orientation. This finding is in support of the literature, which showed that time perception was a mediating variable for the relationship between resilience and academic outcomes (Kruger et al., 2008). Self-efficacy was the primary motivational factor for dropping out or persisting in an online course (Hannah, 2010). Resilience correlated with a future time perspective in the positive direction (Fortunato \& Furey, 2010).

The implication of this current finding is that future orientation does not act alone in predicting success in academia as Zimbardo and Boyd's study implied (Zimbardo \& Boyd, 1999). Therefore, prior attempts at future orientation training to improve academic outcomes (Ferrari et al., 2012; Horstmanshof \& Zimitat, 2007) may not have succeeded for all students because of the influence of resilience on future orientation, which predicted successful academic outcomes (Zimbardo \& Boyd, 1999). This finding may also help explain why some students perform better under the structure of their academic setting, which, in some cases, has shown to promote a future time perspective (Zimbardo \& Boyd, 2008), than others who may experience deadlines and grading rubrics as barriers to continuing their education. Those students high in resilience may be able to use the academic structure to their benefit while those low in resilience may become overwhelmed with an academic profile containing all the classes and milestones necessary to complete a doctorate. Educators interested in improving academic outcomes at the doctoral level may wish to consider evaluating and/or building resiliency skills as well as a future time perspective for students who are not performing well.

Research question Q2: Does attachment style predict time perspective in coursework and dissertation phases of a doctoral distance learning program?

$\mathrm{H} 2_{0}$ : Attachment style will not significantly predict time perspective during the coursework and dissertation phases of a doctoral distance learning program.

H2a: Attachment style will significantly predict time perspective during the coursework and dissertation phases of a doctoral distance learning program. 
Attachment style did predict time perspective. An anxiety attachment style predicted a past-negative time perspective. The implication of this finding is that past-negative time perspective may not have acted alone in past correlation and prediction studies. Past-negative time perspective was associated with dissatisfaction with an academic setting, which may present as a barrier to completing an academic program as past literature has implied (Milfont et al., 2008). Past-negative time perspective may also not have acted alone in predicting pathological gambling and psychiatric conditions (all of which could pose barriers to completing doctoral work) as the work of Hodgins and Engel (2002) found. Attachment styles are believed to shape personality and last into adulthood because of an internal working model mechanism wherein feelings about experiences with the primary caregiver during the first two years of life are stored in the infant's mind and used to predict, interpret, and plan for future events (Bowlby, 1979). Since an anxiety attachment style developed in infancy can last into adulthood, educators should consider providing stress management training to students who may have anxiety attachment styles and past-negative time perspectives in order to decrease anxiety associated with feeling isolated while writing a dissertation online or waiting for online feedback in the absence of face-to-face daily contact with a professor or mentor.

The results of this current study also found that low scores on the anxiety attachment style subscale predicted a past-positive time perspective, which correlated with overall happiness and success (Zimbardo \& Boyd, 1999). Helping students work through some of their past negative events that currently affect them through counseling may help them achieve better academic outcomes.

Low scores on the dependent subscale of the attachment style inventory predicted a future time perspective. These results indicated that students who believed they could not get their needs met by others may have tended to set and achieve their own goals without turning to others for help. Educators may benefit from providing additional structure and/or contact to students who have difficulty working independently or introduce the opportunity for improving individual goal setting and self-organization and motivational skills.

Research question Q3: Does emotional intelligence predict time perspective in coursework and dissertation phases of a doctoral distance learning program?

$\mathrm{H}_{3}$ : Emotional intelligence will not significantly predict time perspective during the coursework and dissertation phases of a doctoral distance learning program.

H3a: Emotional intelligence will significantly predict time perspective during the coursework and dissertation phases of a doctoral distance learning program.

Emotional intelligence did predict time perspective. High emotional intelligence predicted a past-negative time perspective indicating that students who had a negative past may have had to develop emotional intelligence in order to deal with their negative past. This finding was supported by Dunkel and Weber (2010) who found that emotional stability predicted a past-negative time perspective in the positive direction. Low scores in emotional intelligence predicted present-hedonistic and fatalistic time perspectives in this current study. These results were supported by Stolarski et al. (2011) who reported that present-hedonistic time perspective showed a positive correlation to emotional intelligence and a present-fatalistic time perspective showed a negative correlation to emotional intelligence.

The current results indicated that emotional intelligence predicted three out of the five time perspectives studied, so the ability to identify and manage emotions in oneself and others was an important factor in developing and maintaining time perspective, hence academic achievement. These results are supported by the finding that emotional intelligence was important to the development of a balance time perspective (Stolarski et 
al., 2011). These current results may also be related to how students experience their daily activities. If most of their activities during a day lead to feelings of accomplishment, joy, and satisfaction, then students may be more motivated and able to continue in the program. If they feel anxious, angry, depressed, or bad about themselves during their daily experiences, they may have difficulty continuing their educational goals. The results of this study showed that time perspective may not have acted alone in predicting behaviors such as academic outcomes. Stress management, anger management, impulsivity control, emotional expression, and overall emotional regulation interventions in addition to future orientation skill building may help online distance doctoral students who have deficits in these areas to complete their programs. The results of this current study showed that emotional intelligence was an important variable that influences time perspective, hence academic outcomes.

Research question Q4: How will resilience, attachment style, and emotional intelligence interact to predict time perspective during the coursework and dissertation phases of a doctoral distance learning program?

$\mathrm{H}_{4}$ : Resilience, attachment style, and emotional intelligence will not significantly interact to predict time perspective during the coursework and dissertation phases of a doctoral distance learning program.

H4a: Resilience, attachment style, and emotional intelligence will significantly interact to predict time perspective during the coursework and dissertation phases of a doctoral distance learning program. The results of each research question are stated below.

Resilience, emotional intelligence, and attachment style did work together to predict time perspective in doctoral distance learners. Resilience, an independent attachment style, the dissertation phase, marriage, and being male predicted future orientation in this study. These results showed that resilience and independence (the belief that one's needs can be met without frequently depending on others for help) were very important variables in the prediction of a future orientation, which predicted academic success (Zimbardo \& Boyd, 1999). Therefore, resilience and emotional intelligence should be considered as well as future orientation in assessing students for academic success at the doctoral level and kept in mind when setting up additional help for students whose academic performance is substandard.

The dissertation phase also predicted a present-hedonistic time perspective in addition to a future orientation. On first examination, this finding seems counter to what has been reported in the literature by Zimbardo and Boyd (1999). They explained their findings with regards to future oriented undergraduate students as follows. When compared to their peers, future oriented students were more efficient, better at planning, made to do lists, utilized a daily calendar, wore wristwatches, balanced their checkbooks, had more structure and order in their daily lives, and had the most defined goals. Because they tended to have higher standards and experienced pressure from time limits, future oriented students tended to experience more stress than students with other time orientations. They reported having little free time because they filled their time with goal directed activities. Future oriented students' stressful lifestyle paid off in that they had the highest grade point averages and fewer incompletes courses than their classmates. Even though future oriented students felt that it would be healthy to make friends, their hectic overachieving behaviors precluded most of them from making and keeping friends.

Perhaps the online distance students in the dissertation phase of their programs in this study had the time and experience (mean age 45 years) to develop flexibility between a present-hedonistic and future time perspective, allowing them to engage in relationships that foster support in completion of their studies while still maintaining focus on the long-term goal of completing their dissertations. In addition, they may have 
developed a healthy balance between time perspectives. According to the balanced time perspective model proposed by Shirai et al. (2012), an individual should be able to live a full life in the present while also striving for future goals. Therefore, students in this study by the dissertation phase may have developed a balanced time perspective that enabled them to delay gratification in order to work on their dissertations; yet at the same time they may have been able to gain support from others through social interactions and engage in activities other than dissertation writing that allowed them to continue to enjoy life throughout the process of being a doctoral student. This explanation is supported by the finding that graduate students who had a close connection between their present activities and imagined futures tended to value their school work and developed complex knowledge structures necessary for life-long learning (Hilpert et al., 2012).

Effect of limitations on results. The following limitations could have affected the results of this study. All the assessments in the survey were self-report, which can result in a Hawthorne effect. Some participants may have had limited self-awareness and so may have report inaccurately. Participants were instructed to take the survey at home in a comfortable setting without distraction; however, the assessment-taking environment could not be controlled. This researcher made every attempt to solicit participants from different higher educational institutions throughout the US, but if there was a lack of representation from certain geographic locations, there can be no guarantee that the subjects were representative of online, Ph.D. distance learners in the US. The voluntary status of the participants could have produced a bias because of different personality characteristics of those who volunteered and those who did not. The demographic characteristics of the participants were unknown at the time of data collection and the population's demographic characteristics will never be known. Most participants were from middle to upper middle-class in their socio-economic status, which could have also introduced a bias. While the sample size was adequate for logistic regression analysis, more subjects would have strengthened the results. The sample was obtained through purposeful sampling instead of randomization. The ability to generalize the results of this study is therefore limited. All participants were engaged in higher education, so the effects of education on resilience, time perspective, or the development of emotional intelligence could not be examined in this study. The researcher of this nonexperimental quantitative survey used correlations to analyze the type and strength of relationships between variables, so no causal relationship could be established between variables. However, regression analysis can control for possible confounding variables, thereby increasing internal validity. This study proposed to use multiple linear regression analysis but because two assumptions of this type of analysis were violated, normality and homoscedasticity, logistic regressions were used instead. All assumptions were met for logistic regression analysis.

One of the shortcomings of the ZTPI is that there is no positive measure of a present time perspective. Instead, there are two present time perspectives: present-fatalistic and present-hedonistic, both of which have been associated with maladaptive behaviors and emotions (Hodgins \& Engel, 2002; Zimbardo \& Boyd, 1999). What may be missing from this instrument is a present time perspective category that measures the degree to which individuals are focused in the present, fully mindful, and able to block out negative emotions and thoughts that may interfere with a calm, focused, somewhat meditative state in order to be absorbed in a particular task such as working on school work or a dissertation. If such a category of time perspective existed in the overall assessment of time perspective, the results of this study may have been affected. The absence of a "positive" present time perspective may in part account for this study's finding that the dissertation phase of online doctoral programs significantly predicted a present-hedonistic time perspective, a seemingly otherwise 
unusual finding. This result may in part be explained by the following. It may have been that doctoral learners in this phase of their programs were selecting attributes of the present-hedonistic subscale in a positive way instead of a negative way, the latter being the usual way the attributes are viewed. For example, the attribute of choosing to participate in pleasurable activities in the present moment is part of the present-hedonistic time perspective subscale. Participating in pleasurable activities in the present moment usually has a negative connotation because it is often interpreted to mean going to parties, drinking, gambling, having sex, eating, and so forth. But, participating in pleasurable activities in the moment could also mean being able to focus on writing part of a dissertation. A student who considers engaging in writing his or her dissertation pleasurable and all-consuming may report the same value on the Likert-scale as a student who often chooses to indulge in the joy of partying. Both may result in high scores in present-hedonistic time perspective. The presence of a present-focused time perspective subscale in the ZTPI may have separated students who indulge in the pleasure of partying from students who indulge in the pleasure of writing dissertations, hence, giving a more accurate description of the present time perspective and its predictors. Specifically, the dissertation phase may not have predicted a "pure" present-hedonistic time perspective but may have predicted a "present-focused" time perspective.

Relationship to the problem. Zimbardo and Boyd's (1999; 2008) linear unidirectional application of time perspective to academic achievement is limited because of the exclusion of emotional intelligence, resilience, and attachment style that may influence the development and maintenance of time perspective and hence, academic achievement. This current study has demonstrated that emotional intelligence, resilience, and attachment style did predict time perspective in online distance doctoral learners and, therefore, may also be important variables in the prediction of academic achievement. Zimbardo and Boyd's (2008) model of time perspective over the past 30 years has focused mostly on the identification of correlations between time perspective and different human behaviors or studies that identified different human behaviors that were predicted by time perception (Hodgins \& Engel, 2002; Keough et al., 1999; Zimbardo \& Boyd, 1999, 2008; Zimbardo et al., 1997). Their model does not investigate predictors of time perspective, although it states that certain environmental, cultural, geographic, and internal processes can help shape time perspective. Their model does not state how these variables may contribute to the development of time perspective. This current research did show how emotional intelligence, resilience, and attachment style contributed to predicting five different categories of time perspective.

Zimbardo and Boyd's (1999) model of time perspective reported strong positive correlations between future time orientation and academic success (Zimbardo \& Boyd, 1999), but it does not address the question of why the structure of school helps some students succeed while it hinders others. Certain students can learn to thrive under the structure of an academic setting (move from a past-negative or present-hedonistic time perspective bias to a future orientation) and others cannot. Results of this current study, that resilience and attachment style were important factors in the prediction of future orientation, can answer this question. Students who are resilient and can work independently may be able to develop more of a future orientation by using the limits, boundaries, and structure imposed by school to their advantage, while those without resilience and who depend on others to meet their needs may not be able to "rise to the occasion" given the structure of an academic setting or interventions to promote a future orientation. Students with other time perspectives that have been shown to inhibit academic success (present-hedonistic, present-fatalistic) (Boyd \& Zimbardo, 2005; de Volder \& Lens, 1982; Harber et al., 2003) may not be able to benefit from future time orientation training or 
time management because emotional intelligence may be affecting their time perspective as indicated by the results of this study: low scores in emotional intelligence predicted both present-hedonistic and fatalistic orientations. For these students low in emotional intelligence, stress management and emotional regulation may be more beneficial than future orientation training alone.

Zimbardo and Boyd's theory of time perspective (1999; 2008) does not analyze the phenomenon of flexibility of time perspective that some individuals possess and others do not (remaining fixed in a particular bias instead). This current study showed that the less structured dissertation phase of distance online learning predicted both future and hedonistic time perspectives. Based on literature that reported that a future orientation correlated with academic success (Horstmanshof \& Zimitat, 2007; Peetsma, 1994; Zaleski, 1987; Zimbardo \& Boyd, 1999), the prediction of future orientation by the dissertation phase was expected. Given the literature that reported that hedonistic time perspective correlated with poor academic outcomes (Boyd \& Zimbardo, 2005; de Volder \& Lens, 1982; Harber et al., 2003), the prediction of hedonistic time perspective by dissertation phase was unexpected, but can be explained if students in the dissertation phase had developed flexibility to move between time perspectives at will as necessitated by environmental demands.

In addition, Zimbaro and Boyd's (1999) theory claims that time perception continually develops and changes but does not explain which aspects of structure and discipline affect time perspective or its plasticity. Students choose online distance learning programs because they offer a flexible schedule (Parsad \& Lewis, 2008). The freedom to study at convenient times sounds desirable, yet more students drop out of online distance learning programs than do those enrolled in classical programs (Cockran, 2009) due to an inability to manage time or anticipate the amount of discipline necessary to complete assignments without the structure of deadlines (Cockran, 2009; Street, 2010). Other reasons related to time may exist that make it difficult for students to complete online distance doctoral programs. Students may not be able to balance work, leisure, school work, responsibilities in other roles, and sleep, necessary for mental and physical health (Christiansen \& Matuska, 2006).

Certain students require a high degree of structure in order to be productive in academic pursuits, while others feel trapped and stifled with too much structure and too many rules. Coursework tends to be more structured than the dissertation phase, so some students may do better during the coursework phase versus the dissertation phase or vice versa. Other factors such as emotional intelligence, resilience, and attachment styles were found to be influential factors in the development and maintenance of time perspective in this current study and so are important factors in the consideration of online distance doctoral learning outcomes.

The manner in which resilience, emotional maturity, and attachment style may predict time perception was unknown before this current study. This current study's results advance Zimbaro and Boyd's (1999) theory of time perspective by identifying predictors and prediction models of time perspective. The presence of predictors indicate that previous research was incomplete in its explanation of the relationship between time perspectives and human behaviors, such as academic achievement, because of the contribution of emotional intelligence, resilience, and attachment style to time perspective. Results also indicate the need to include these variables in prediction models of attrition rates of online doctoral programs. The guiding theoretical pursuit of this research was to ascertain whether emotional intelligence, attachment style, and resilience operate differently to predict time perception in a structured (coursework) versus the less structured (dissertation) phase of doctoral distance learning. Results showed that subjects in the dissertation phase (low amount of structure) of their online distance doctoral learning program had 2.72 times greater odds of having a present hedonistic 
time perspective and 5.26 times greater odds of having a future time perspective orientation than their counterparts in the course work phase (high amount of structure) of their online distance doctoral learning programs.

Without the consideration of the contribution of emotional intelligence, resilience, and attachment style to the development and maintenance of time perspective, studies that have reported that time perspective predicted academic success are incomplete. Flexibility between time perspectives cannot be addressed. Interventions created to develop future time perspectives in students entering the dissertation phase of their programs may not be complete. Predictions of success or failure in online distance learning doctoral programs cannot be accurately determined. Therefore, the results of this current study can fill a gap in the literature by identifying factors that develop and work together to maintain time perspective. These factors are necessary to predict more accurate academic outcomes, address the phenomena of time perspective flexibility, and guide educators in setting up specific individualized interventions for academic success.

The results of this current study have added important information to the literature of time perspective based on the model purported by Zimbardo and Boyd $(1999 ; 2008)$ as noted above. The current results are important to all time perspective studies (not just to the field of academia) because of the many correlations between time perspective and other human behavior reported in the literature and should be considered as factors that shape and possibly maintain time perspective biases, balancing, and flexibility throughout the life span.

Relationship to the purpose. The purpose of this nonexperimental quantitative survey study was two-fold. First, it was used to identify what predictive role emotional intelligence, attachment style, and resilience (predictor variables) had on time perception (criterion variable) in doctoral distance learners, since each of these variables have been shown to influence behavior and relate to time perspective (Fortunato \& Furey, 2010; Laghi et al., 2009; Monahan et al., 2010; Padawer et al., 2007). The results showed that emotional intelligence, attachment style, and resilience each individually predicted time perspective in doctoral distance learning students in the course work phase and dissertation phase of their programs. Emotional intelligence individually predicted time perspective. High scores on the emotional intelligence scale predicted a past-negative orientation while low scores on the emotional intelligence scale predicted present-hedonistic and fatalistic time perspectives. Resilience also individually predicted time perspective. High scores on the resilience scale predicted a future orientation. Attachment style individually predicted time perspective. An anxiety attachment style predicted a past-negative time perspective. Low scores on the anxiety subscale predicted a past-positive time perspective. Low scores on the dependent attachment subscale predicted a future orientation. Program phase predicted time perspective. The dissertation phase predicted present-hedonistic and future orientations.

Second, the study was used to ascertain how emotional intelligence, attachment style, and resilience (predictor variables) interacted to predict time perception (criterion variable). The results showed that emotional intelligence, attachment style, and resilience worked together to predicte time perspective in doctoral distance learning students in the course work phase and dissertation phase of their programs. Past-negative time perspective was predicted by an anxiety attachment style and high scores on emotional intelligence. Past-negative time perspective was predicted by a low score on the anxiety attachment style subscale. Present-hedonistic time perspective was predicted by low emotional intelligence and the dissertation phase. Present-fatalistic time perspective was predicted by low emotional intelligence. Future time perspective was predicted by high levels of resilience, independence, the dissertation phase, marriage, and being male as 
opposed to female. The results provide clarification to and expand upon Zimbardo and Boyd's (1999) theory of time, which did not identify or study predictors of time perspective. The results may be used to address the high attrition rate of online doctoral programs as noted below.

Significance. The results of this study have both theoretical significance and clinical application. This study's most important contribution was to fill a gap in Zimbardo and Boyd's (1999; 2008) model of time that purports that time perspective is related to and in some cases predicts certain human behaviors. The past 30 years of research have been aimed at establishing this linear approach and ascertaining which behaviors result from which time perspective. The current study expanded their model by identifying other variables (emotional intelligence, resilience, attachment style, level of structure) that may be responsible for establishing, altering, and sustaining time perspective throughout the life span, thereby explaining questions that the model could not previously account for. One such question in the area of academia being, why does the structure of school help some individuals succeed while it hinders others? More specifically, why does school help discipline some students (e.g., by helping them move from a present-hedonistic to a more future orientation) but not all students? This current study provides additional insight into the phenomenon of flexibility of time perspective that some individuals seem to possess while others do not. Instead, they remain fixed in a particular bias. On a broader scale, it opens the door for other researchers to investigate these and other variables that may be responsible for influencing time perception, which in turn may influence many other human behaviors.

The application of time perception to academia is of particular interest because distance learning programs are becoming more prevalent (Allen \& Seaman, 2011) but have a higher attrition rate than brick and mortar schools (Cockran, 2009; Council of Graduate Schools, 2008) and time perspective has been shown to predict success and failure in academia (Boyd \& Zimbardo, 2005; Harber et al., 2003; Zimbardo \& Boyd, 1999; Horstmanshof \& Zimitat, 2007). The growth rate of online learners from 2009 to 2010 was $10 \%$ compared to a $1 \%$ growth rate in classical higher education during the same time frame according to The Sloan Foundation's report (Allen \& Seaman, 2011). However, the attrition rate for all but dissertation distance learners is $70 \%-80 \%$ (Flood, 2002; Nichols \& Levy, 2009), which is higher than the 50\% reported for traditional learners in brick and mortar institutions (Cockran, 2009; Council of Graduate Schools, 2008).

The high attrition rate for distance learners has been attributed in part to poor time management skills (Barbour \& Reeves, 2009; Cockran, 2009; Long et al., 2009; Street, 2010). Time management skills are predicated upon the perception of time (Zimbardo \& Boyd, 1999; 2008). Future oriented time perspective was consistently related to positive academic outcomes across many studies. Zimbardo, Boyd, and co-workers found that people who were the most academically successful tended to have a future orientation, allowing them to see ahead, delay gratification, set goals, and manage their time, whereas present oriented individuals had the highest college attrition rate (Boyd \& Zimbardo, 2005; de Volder \& Lens, 1982). Individuals who scored high in future orientation were more punctual for class, apt to complete homework before due dates, inclined to begin assignments on time, and skilled in time management than their classmates (Harber et al., 2003). The same study reported significant gender differences; future oriented females began their assignments before their male counterparts. Future oriented college students also tended to utilize successful learning strategies such as reproductive and deep-level cognitive processing more often than students with other time orientations (Horstmanshof \& Zimitat, 2007). In addition, persistence (Peetsma, 1994; Zaleski, 1987), level of engagement (Peetsma, 1994), satisfaction (Zaleski, 1987), number of hours spent in school work (Peetsma, 1994) and grade point average (Zimbardo \& Boyd, 1999) were related to future orientation among college 
students. Future orientation was also associated with academic achievement in high school learners (Anderman et al., 1999; de Volder \& Lense, 1982; Groth, 1973; Honora, 2002; Shell \& Husman, 2001). Conversely, there was a negative correlation between past-negative and students' report of satisfaction with their educational institutions (Horstmanshof \& Zimitat, 2007). Students oriented to the present were late signing up for, initiating, and completing assignments, so they tended to receive more incompletes and poorer grades, were assigned to special education classes more often, and dropped out of school more frequently than did their counterparts (Harber et al., 2003). The poorest grades were characteristic of fatalists, followed by hedonists. The results of this study have both theoretical significance and clinical application.

Contribution to the literature. The presence of predictors indicates that previous research was incomplete in its explanation of the relationship between time perspectives and human behaviors, such as academic achievement, because of the contribution of emotional intelligence, resilience, and attachment style to time perspective. Results also indicated the need to include these variables in prediction models of attrition rates of online doctoral programs. Most studies on time perception and human behavior (Harber et al., 2003; Horstmanshof \& Zimitat, 2007; Zimbardo \& Boyd, 1999, 2008) including academic achievement (e.g., grade point average, number of hours spent studying, timely completion of assignments and goals) have been either correlations or investigations of how time perception predicted behavior. Academic achievement has been shown to have a positive correlation with a bias for future time orientation and a negative correlation with certain past and present orientations (Boyd \& Zimbardo, 2005; Harber et al., 2003; Zimbardo \& Boyd, 1999). These relationships were linear in that they examined correlations between time perspective and academic achievement instead of considering a multiple variables or models of prediction. Research that has examined how time perspective predicted specific behaviors (academic achievement) may be considered unidirectional in nature because it has omitted the study of predictors of time perception and, hence, their possible influence on human behaviors such as academic achievement. Most of the literature describing the relationship of time perspective on academic achievement was performed on traditionally taught college or high school students, not doctoral students enrolled in online distance learning programs. The results of this current research addresses this gap in the literature. The results of this current study indicate that time perspective does not act alone in predicting human behaviors such as academic success, as has been noted in the last 30 years of literature, but that other factors, such as emotional intelligence, attachment style, resilience, and level of structure shape the development and sustainability of time perspective.

\section{Recommendations}

\section{Practical Applications}

The identification of time perception predictors and their relationship to doctoral distance learners in the coursework versus dissertation phase of learning is of clinical interest because of the high attrition rate that occurs during the latter and the potential to change maladaptive time perspectives into those that better support the ability to complete an online dissertation. Many students (over 6.1 million in the fall of 2010 (Allen \& Seaman, 2011)) have pursued distance learning. The growth rate of online learners from 2009 to 2010 was $10 \%$ compared to a $1 \%$ growth rate in classical higher education during the same time frame according to the same report. Many of these students chose distance learning doctoral programs over traditional classroom instruction because they could complete their coursework and dissertation during time that was convenient for them instead of attending class in a set place and during a particular time (Kvavik \& Caruso, 2005). Asynchronous 
distance learning programs afforded students the leeway to continue to participate in various life roles that did require strict adherence to a set schedule, such as work or child care, while engaging in school work during free time (Aragon \& Johnson, 2013). A flexible schedule was one of the top four most common factors influencing distance learning program enrollment as reported by the US Department of Education, Institute of Education Sciences, National Center for Educational Statistics (NCES) (Parsad \& Lewis, 2008). Even though the ability to study whenever a student desires sounds convenient, more students fail to complete distance learning programs than traditional classroom education (Cockran, 2009) due to an inability to manage time or anticipate the amount of discipline necessary to turn in assignments without an imposed deadline (Cockran, 2009; Street, 2010).

There may be additional reasons related to time that make it difficult for students to complete distance learning doctoral programs. Students may be not being able to maintain a balance of time in the areas of work, leisure, responsibilities, school work, and sleep, which has been found to be important for maintaining stress reduction, psychological need satisfaction, and role balance necessary for physical and mental health (Christiansen \& Matuska, 2006). Another may be related to how students experience their daily activities. If a majority of their daily time is spent in activities that cause them to feel anxious, depressed, angry, or bad about themselves, they may experience difficulty continuing the program. If the number of hours in a typical day is predominantly filled with activities that result in students feeling positive affects, they may be more motivated to achieve their educational goals. Some students may require more structured time. Coursework tends to be more structured than the research involved in a dissertation. Therefore, some students may perform better during the coursework phase of a doctorate than the dissertation phase or vice versa if they perform more efficiently given unstructured time. Therefore, other factors such as emotional intelligence, resilience, and attachment styles may be influencing time perspective.

The results of this study have direct application to the field of online distance education. Resilience and independence predicted a future time orientation in doctoral distance learners in this study. Past literature has established that a future time perspective predicted academic success at the high school (Anderman et al., 1999; de Volder \& Lens, 1982; Groth, 1973; Honora, 2002; Shell \& Husman, 2001) and college levels (Boyd \& Zimbardo, 2005; de Volder \& Lens, 1982; Harbor et al., 2003; Horstmanshof \& Zimitat, 2007; Peetsma, 1994; Zaleski, 1987; Zimbardo \& Boyd, 1999). Previous studies have administered various interventions to students who scored low in future orientation and performed poorly in school in attempt to increase their future orientation and hence, academic performance (Ferrari et al., 2012; Hosrtmanshof \& Zimitat, 2007). Many of the students in these studies did show improvement in future orientation but not all of them demonstrated scholastic improvements. It may be that increasing scores on future time perspective is not enough to increase academic success for all students because other factors such as resilience and the ability to rely on oneself instead of others to work independently may predict future orientation, as demonstrated in this study. By providing interventions that promote resilience and independence as well as future orientation, more students may be able to show academic improvements. For students who may not be able to learn to work more independently, the learning institution may provide more structure, especially in the dissertation phase of the doctoral program so students can benefit from an imposed regime that organizes them throughout the many steps of writing a dissertation.

In this study, low emotional intelligence predicted a present time perspective. Previous literature has established that present time perspectives (both fatalistic and hedonistic) predicted poor academic outcomes (Harber et al., 2003). Therefore, online distance learning doctoral programs may benefit from devising methods 
for students to better manage their emotions (stress, frustration, fear, anxiety, anger, depression, loneliness, isolation) that may get in the way of completing their studies. If monitored and used appropriately, online forums may help students express their emotions, relate to others' emotions, and gain suggestions and motivation from other students on how to move beyond their emotions and return to productive work. Online communication via forums may help distance learning students feel less isolated in their emotional struggles, although, for some, reading and posting about their barriers may fuel their negative emotions and make their struggles worse. For these students, seminars on emotional regulation and productivity could be made available online for these students to participate anonymously.

In this study, an anxiety attachment style predicted a past-negative time perspective. A past-negative time perspective correlated with students' dissatisfaction with their institutes of higher educational learning (Horstmanshof \& Zimitat, 2007). Feelings of dissatisfaction can be a barrier to learning. An anxiety attachment style in adults is marked by a fear of abandonment. Online distance doctoral learners with past-negative time perspectives who express dissatisfaction with aspects of their learning institutions may benefit from increased communication from their professors, mentors, and academic advisors. Increasing the frequency of communication, while intending to address academic impasses, may also serve to decrease anxiety and, hence, increase satisfaction with school and result in a return to active learning.

Admissions criteria are important considerations when determining which students are most probable to do well while in a particular program. Lysaght, Donnelly, and Villeneuve (2009) evaluated how preadmission criteria correlated with retention in an academic program. Their results indicated that predictive factors in academic outcomes were not necessarily scholastic-based. Instead, certain personal characteristics should be used as basis for admission boards because they showed positive correlation with student scholastic success. This move away from the efficacy of classic admission criteria showed that there is a valid need for non-traditional acceptance criteria. One such non-traditional variable that may determine success in higher level academia is the extent to which students can manage their time. Beaudoin (2013) found that time management was the second most important factor in academic achievement next to self-motivation. Time management is predicated upon the perception of time and time perspective is predicted by resilience, emotional intelligence, and attachment style as shown by this study. Therefore, assessments of time perspective, resilience, emotional intelligence, and attachment style may be used to evaluate potential doctoral students to help determine which learning environment may be best for them: classical versus online, asynchronous versus synchronous, or a structured versus a more independent dissertation phase. In addition to or instead of assessing students upon admission, learning institutions may assess students that are not performing to academic standards in order to understand how best to help them (i.e., provide them with seminars on time management, emotional regulation, stress management, overcoming barriers, and working independently).

Elements necessary to provide internal structure required for success in academic pursuits include the ability to set and achieve long-term goals through persistence and focus. Academic goal attainment requires giving up immediate gratification for long-term gratification, engaging in school related activities, beginning assignments on time, managing time, utilizing deep-level cognitive processing, persisting in the face of adversity and overcoming barriers, and possessing a sense of self-efficacy. These skills do not develop in present-fatalistic oriented individuals who doubt they have the capacity to improve their circumstances or present-hedonistic individuals who are impulsive and live for the moment (Zimbardo \& Boyd, 2008). The coursework phase of doctoral programs may be providing some of these elements through its imposed structure. 
When some of this structure is removed during the dissertation phase, learners who cannot provide their own structure may precipitate attrition. This may be the reason that the results of this study showed that the dissertation phase of the doctoral program predicted a future time perspective. Perhaps the students in this study did not need to be future oriented in the course work phase (since the course work phase did not significantly predict a future time perspective) because the course work phase of their program may have been more structured. Students in the dissertation phase in this study may have needed to become more future oriented because of the lack of structure in this phase. Students who did not become more future oriented may have dropped out at the ABD phase of their programs.

\section{Further Research}

This study and its results present many opportunities for further research. With a greater sample size, a randomized study with the same methodology would be possible and result in a more rigorous study. A qualitative study examining the experience of time in online distance doctoral students would add rich, in-depth information about how each participant in the different program phases experienced time. The results of such a qualitative study may help educators better understand how to help doctoral students complete their degrees. Or, a mixed method study comparing results of quantitative and qualitative natures of the experience of time and possible variables that influence the development and maintenance of time perspective while engaged in doctoral online studies would be of interest. This current study was cross-sectional in nature so did not consider change in time perspective or its predictors over time in the same subjects. Such a study that involved a longitudinal examination of how predictors of time perspective and time perspective itself changes with the amount of structure would be interesting.

This current study examined the prediction of five time perspective categories individually. It did not examine each participant's time perspective profile. A time perspective profile is the combination of all five time perspective categories per participant (i.e., an individual score for each subscale is generated per participant but considered as a whole). Considering participants' overall time perspective profiles would give a broader picture of each participant's time perspective. For example, a person may be high in past-negative, moderately high in present-hedonistic, low in present- fatalistic, and high in future orientation. Such a person may have had a negative past and may still be thinking about it, yet does not have a fatalistic present orientation so may have worked through enough of the past negative experiences or gained coping skills as a result in order to not be fatalistic in the present. This person may be able to enjoy him or herself in the present (hedonistic) while maintaining future goal-directed activity. Such an analysis would be possible using the ZTPI and may enhance interpretation of predictors of such time perspective clusters.

This study also did not consider time perspective biases. Instead, it considered a time perspective to fall into a certain category based on whether or not subjects scored above or below the mean for each subscale of time perspective. A future study may be based on time perspective biases. In order for a subject to have a biased time perspective, he or she would have to score in the top quartile for only one of the five time perspective subscales while scoring below the top quartile for the other four. Subjects not meeting this requirement would not be considered to have a time perspective bias, and, hence, be eliminated from a study examining predictors of time perspective biases. Such a study of biases would add more information to the current study.

A study of the flexibility of time perspective (i.e., variables that change or alter time perspective) in adult online distance doctoral learners would be a very valuables follow-up study to this current research. A project 
of this sort would help identify factors that could enhance future time perspective while decreasing past-negative, present-hedonistic, and present-fatalistic time perspectives in order to approximate a "successful" time perspective profile. Flexibility is also important for adaptation. A study of this sort may also elucidate the achievement of a balanced time perspective, which has also been shown to be necessary for success and happiness in life (Zimbardo \& Boyd, 1999; 2008).

Studies that examine each time perspective's developmental history and how time perspectives are strengthened, weakened, learned, and maintained over time is necessary. Research investigating the difference between individuals with balanced time perspectives and those with biases may also be helpful to online distance doctoral learners and their educators. This study examined three predictors (attachment style, resilience, and emotional intelligence) of time perspective in different phases of doctoral programs but there may be other predictors such as culture, location, personal experiences, religion, climate, family, peers, work, school, social models, and others that may function together or separately to develop, maintain, and/or alter time perspective.

\section{Conclusions}

In this study, attachment style, resilience, and emotional intelligence individually predicted time perspective and worked together to predict time perspective in online distance learners in the course work and doctoral phase of their programs. Future time perspective was predicted by resilience, $p=0.005$; independent attachment style, $p=0.008$; dissertation phase, $p=0.006$; marital status (married), $p=0.020$; and gender (male), $p=0.010$. Present-hedonistic time perspective was predicted by low emotional intelligence, $p=0.034$ and dissertation phase, $p=0.037$. Present-fatalistic time perspective was predicted by low emotional intelligence, $p=0.015$. Past-positive time perspective was predicted by low anxiety attachment scores, $p=$ 0.019. Past-negative time perspective was predicted by an anxiety attachment style, $p=0.034$ and high emotional intelligence, $p=0.007$.

Results indicated that the past 30 years of research involving predictions of human behavior by time perspective and correlation studies of the relationship between time perspective and human behavior were incomplete since they did not consider the influence of attachment style, resilience, and emotional intelligence on time perspective. Much of the literature in academia involving time perspective has focused on the relationship between academic outcomes and time perspective or the prediction of successful academic outcomes by future time perspective. The results of these studies generated many further questions, which the studies could not answer because they were incomplete. The results of this current study addressed these questions and added valuable information about the development and maintenance of time perspective to Zimbardo and Boyd's (1999; 2008) model of time perspective, thereby expanding their theory.

The identification of significant predictors of time perspective provides information that can help educators and administrators of online distance doctoral programs set up and implement interventions that can more accurately help students finish their doctoral programs. Academic educators of doctoral studies may wish to use the results of resilience, attachment, emotional intelligence, and time perspective measures to aid students in their decision of what type of learning environment may best suit their learning needs before committing to a program. Most of the measures used in this study can be found online and taken, scored, and interpreted by students anonymously on their own. 


\section{References}

Agresti, A., \& Franklin, C. A. (2013). Statistics: The art and science of learning from data (3rd ed.). Boston, M.A.: Pearson Education.

Allen, I. E., \& Seaman, J. (2011). Going the distance: Online education in the United States. Newburyport, M.A.: Sloan Consortium.

Anderman, E. M., Anderman, L. H., \& Griesinger, T. (1999). The relation of present and possible academic selves during early adolescence to grade point average and achievement goals. Elementary School Journal, 1000, 3-17. doi:10.1086/461940

Angelino, L. M., \& Natvig, D. (2009). A conceptual model for engagement of the online learner. The Journal of Educators Online, 6, 1-19. Retrieved from http://www.thejeo.com/Archives/Volume6Number1/Angelinoetalpaper.pdf

Apostolidis, T., \& Fieulaine, N. (2004). The Zimbardo Time Perspective Inventory (ZTPI). European Review of Applied Psychology/Revue 54, 207-217. doi:10.1016/j.erap.2004.03.001

Aragon, S. R., \& Johnson, E. S. (2013). Factors influencing completion and noncompletion of community college online courses. American Journal of Distance Education, 22(3), 146-158. doi: 10.1080/08923640802239962

Aronowitz, T., \& Morrison-Beedy, D. (2004). Resilience to risk-taking behaviors in impoverished African American girls: The role of mother-daughter connectedness. Research in Nursing \& Health, 27, 29-39. doi:10.1002/nur.20004

Azar, S. (2013). Impact of time management training on Pakistani corporate sector employees. Journal of Basic and Applied Scientific research, 3(4), 476-482. Retrieved from http://www.textroad.com/pdf/JBASR/J.\%20Basic.\%20Appl.\%20Sci.\%20 Res.,\%203(4)476-482,\%202013.pdf

Ballard, D. I., \& Seibold, D. R. (2006). The experience of time at work: Relationship to communication load, job satisfaction, and interdepartmental communication. Communication Studies, 57, 317-340. doi:10.1080/10510970600845974

Bandura, A. (1977). Social learning theory. Englewood Cliffs, N.J.: Prentice-Hall.

Barber, L. K., Munz, D. C., Bagsby, P. G., \& Grawitch, M. J. (2009). When does time perspective matter? Self-control as a moderator between time perspective and academic achievement. Personality and Individual Differences, 46(2), 250-253. doi: 10.1016/j.paid.2008.10.007

Barbour, M. K., \& Reeves, T. C. (2009). The reality of virtual schools: A review of the literature. Computers and Education, 52, 402-416. doi:10.1016/j.compedu.2008.09.009

Beaudoin, M. (2013). The evolving role of the instructor in the digital age. In Y. Kats (Ed.), Learning management systems and instructional design: Best practices in online education (pp. 233-247). Hershey, P.A.: Idea Group Inc.

Beauvais, A. M., Brady, N., O’Shea, E. R., \& Griffin, M. T. (2011). Emotional intelligence and nursing performance among nursing students. Nurse Education Today, 31, 396-401. doi:10.1016/j.nedt.2010.07.013

Belkin, D. (2013, February 14). Web classes grapple with stopping cheats. The Wall Street Journal. Retrieved from http://online.wsj.com/article/SB10001424127887324906004578292361415395332.html

Berlin, L. J., Cassidy, J., \& Appleyard, K. (2008). The influence of early attachments on other relationships. In J. Cassidy, \& P. Shaver (Eds.), Handbook of attachment: Theory, research and clinical applications (pp. 333-347). London, England: Guilford.

Block, R. A. (Ed.) (1990). Cognitive models of psychological time. Hillsdale, N.J.: Erlbaum.

Bodie, G. D. et al. (2011). Explaining the impact of attachment style on evaluations of supportive messages: A dual-process framework. Communication Research, 38, 228-247. doi:10.1177/0093650210362678

Boniwell, I., \& Zimbardo, P. G. (2003). Time to find the right balance. The Psychologist, 16, 129-130. doi:10.1037/0022-3514.77.6.1271

Boniwell, I., \& Zimbardo, P. G. (2004). Balancing time perspective in pursuit of optimal functioning. In P. A. Linley, \& S. Joseph (Eds.), Positive psychology in practice (pp. 165-178). Hoboken, N.J.: Wiley.

Boniwell, I., Osin, E., Linley, P. A., \& Ivanchenko, G. V. (2010). A question of balance: Time perspective and well-being in British and Russian samples. The Journal of Positive Psychology, 5(1), 24-40. doi: 10.1080/17439760903271181

Borden, K. S., \& Abbott, B. (2005). Research and design methods: A process approach (6th ed.). Mc-Graw-Hill Companies, New York.

Bowers, M., Freyman, N., McLellan, E., Paxton, B., \& Spiegel, J. (2013). Online and traditional lectures: Evaluating the effects of social presence and learner control. Gemstone Team ONLINE. Retrieved from: http://drum.lib.umd.edu/bitstream/1903/13885/1/ONLINE\%20Final\%20Thesis.pdf

Bowlby, J. (1979). The making and breaking of affectional bonds. London, England: Tavistock. 
Boyd, J. N., \& Zimbardo, P. G. (2005). Time perspective, health and risk taking. In A. Strahman, \& J. Joireman (Eds.), Understanding behavior in the context of time: Theory, research and applications (pp. 85-107). Mahwah, N.J.: Erlbaum.

Boyd, V. S., Gast, L., Hunt, P., Mitchell, A., \& Wilson, W. (2010). Seniors who leave college during a semester (Research Report 2000-2010). Retrieved from http://www.counseling.umd.edu/Infodata/srsleavecoll.pdf

Breakwell, G. M., Hammond, S., Fife-Schaw, C., \& Smith, J. A. (2006). Research methods in psychology. London: Sage Publications.

Buhusi, C. V., \& Meck, W. H. (2009). Relative time sharing: New findings and an extension of the resource allocation model of temporal processing. Philosophical Transactions of the Royal Society: Biological, 364, 1875-1885. doi:10.1098/rstb.2009.0022

Campbell, L. A., \& Bryant, R. A. (2007). How time flies: A study of novice skydivers. Behavior Research and Therapy, 45, 1389-1392. doi:10.1016/j.brat.2006.05.011

Carelli, M. G., Wiberg, B., \& Wiberg, M. (2011). Development and construct validation of the Swedish Zimbardo Time Perspective Inventory. European Journal of Psychological Assessment, 27(4), 220-227. doi: 10.1027/1015-5759/a000076

Carstensen, L. L., Isaacowitz, D. M., \& Charles, S. T. (1999). Taking time seriously: A theory of socioemotional selectivity. American Psychologist, 54, 165-181. doi:10.1037/0003-066X.54.3.165

Cemaloglu, N., \& Filiz, S. (2010). The relation between time management skills and academic achievement of potential teachers. Educational Research Quarterly, 33(4), 3-23. Retrieved from http://eric.ed.gov/?id=EJ935088

Chambon, M., Droit-Volet, S., \& Niedenthal, P. M. (2008). The effect of embodying the elderly on time perception. Journal of Experimental Social Psychology, 44, 672-678. doi:10.1016/jjesp.2007.04.014

Christiansen, C. H., \& Matuska, K. M. (2006). Lifestyle balance: A review of concepts and research. Journal of Occupational Science, 13(1), 49-61. doi:10.1080/14427591.2006.9686570

Cockran, S. (2009). Why a high rate of drop out for distance learning students? Retrieved from www.brighthub.com/education/online-learning/articles33547.aspx

Collins, N. L., \& Read, S. J. (1990). Adult attachment, working models, and relationship quality in dating couples. Journal of Personality and Social Psychology, 58, 644-663. doi:10.1037//0022-3514.58.4.644

Corral-Verdugo, V., Fraijo-Sing, B., \& Pinheiro, J. D. Q. (2006). Sustainable behavior and time perspective. Present, past, and future orientations and their relationship with water conservation behavior. Interamerican Journal of Psychology, 40, 139-147. doi:10.1016/j.erap.2005.09.002

Côté, S., DeCelles, K. A., McCarthy, J. M., Van Kleef, G. A., \& Hideg, I. (2011). The Jekyll and Hyde of emotional intelligence: Emotion-regulation knowledge facilitates both prosocial and interpersonally deviant behavior. Psychological Science, 22, 1073-1080. doi:10.1177/0956797611416251

Coudin, G., \& Lima, M. L. (2011). Being well as time goes by: Future time perspective and well-being. International Journal of Psychology and Psychological Therapy, 11(2), 219-232. Retrieved from http://www.ijpsy.com/volumen11/num2/294/being -well-as-time-goes-by-future-time-ES.pdf

Council of Graduate Schools. (2008). Ph.D. completion and attrition: Analysis of baseline demographic data from the Ph.D. completion project, executive summary. Retrieved from http:/www.phdcompletion.org/information/Executive_Summary_ Demographics_Book_II.pdf

Creswell, J. W., \& Plano Clark, V. L. (2011). Designing and conducting mixed methods research (2nd ed.). Thousand Oaks, C.A.: SAGE Publications.

Cretu, R. (2012). A confirmatory approach of the structure of Zimbardo's time perspective. Concept, Cognition, Brain, Behavior: An Interdisciplinary Journal, (16)4, 481-494. Retrieved from https://erms.tourolib.org/url/http://search.ebscohost.com/login. aspx?direct $=$ true $\& \mathrm{db}=\mathrm{a} 9 \mathrm{~h} \& \mathrm{AN}=84429774 \&$ site $=$ ehost-live

D’Alessio, M., Guarino, A., de Pascalis, V., \& Zimbardo, P. G. (2003). Testing Zimbardo's Stanford Time Perspective Inventory (STPI)—Short Form: An Italian study. Time \& Society, 12, 333-347. doi:10.1177/0961463X030122010

Darley, J. M., \& Batson. (1973). From Jerusalem to Jericho: A study of situational and dispositional variables in helping behavior. Journal of Personality and Social Psychology, 27, 29-40. doi:10.1037/h0034449

de Bilde, J., Vansteenkiste, M., \& Lens, W. (2011). Understanding the association between future time perspective and self-regulated learning through the lens of self-determination theory, learning and instruction. Learning and Instruction, 2, 332-344. doi:10.1016/j.learninstruc.2010.03.002

De La Rosa, M., Dillon, F., Rojas, P., Schwartz, S., \& Duan, R. (2010). Latina mother-daughter dyads: Relations between attachment and sexual behavior under the influence of alcohol or drugs. Archives of Sexual Behavior, 39, 1305-1319. doi:10.1007/s10508-009-9498-2 
de Volder, M. L., \& Lens, W. (1982). Academic achievement and future time perspective as a cognitive-motivational concept. Journal of Personality and Social Psychology, 42, 566-571. doi:10.1037/0022-3514.42.3.566

Díaz-Morales, J. F. (2006). Factorial structure and reliability of Zimbardo Time Perspective Inventory. Francisco Psicothema, 18, 565-571. Retrieved from http://ehis.ebscohost.com/eds/detail?vid=4\&hid=6\&sid=bab16498-d090-474a-9edb-7b0907793480 $\% 40$ sessionmgr15\&bdata=JnNpdGU9ZWRzLWxpdmU\%3d\#db=edswss\&AN=000239104400036

Droit-Volet, S., \& Gil, S. (2009). The time-emotion paradox. Philosophical Transactions of the Royal Society Biological Sciences, 364, 1943-1953. doi:10.1098/rstb.2009.0013

Droit-Volet, S., \& Meck, W. H. (2007). How emotions color our perception time. Trends Cognitive Science, 11, 504-513. doi:10.1016/j.tics.2007.09.008

Dunkel, C. S., \& Weber, J. L. (2010). Using three levels of personality to predict time perspective. Current Psychology, 29(2), 95-103. doi:10.1007/s12144-010-9074-x

Eagleman, D. M., \& Pariyadath, V. (2009). Is subjective duration a signature of coding efficiency? Philosophical Transactions of the Royal Society: Biological, 364, 1841-1851. doi:10.1098/rstb.2009.0026

Einstein, A. (1931). Relativity: The special and general theory (R. W. Lawson, Trans.). New York: Crown.

Faul, F., Erdfelder, E., Buchner, A., \& Lang, A. G. (2009). Statistical power analyses using G*Power 3.1: Tests for correlation and regression analyses. Behavior Research Methods, 41, 1149-1160. doi:10.3758/BRM.41.4.1149

Ferrari, L., Nota, L., \& Soresi, S. (2012). Evaluation of an intervention to foster time perspective and career decidedness in a group of Italian adolescents. The Career Development Quarterly, 60(1), 82-96. doi: 10.1002/j.2161-0045.2012.00007.x

Flood, J. (2002). Read all about it: Online learning facing 80\% attrition rates. Turkish Online Journal of Distance Education, 3(2). Retrieved from http://tojde.anadolu.edu.tr/tojde6/articles/jim2.htm

Fortunato, V. J., \& Furey, J. T. (2010). The theory of mindtime: The relationships between future, past, and present thinking and psychological well-being and distress. Personality and Individual Differences, 50(1), 20-24. Retrieved from http://www.diva-portal.org

Gall, M., Borg, W., \& Gall, J. (2007). Educational research: An introduction. White Plains, N.Y.: Longman, USA.

Gao, Y. (2011). Time perspective and life satisfaction among young adults in Taiwan. Social Behavior and Personality, 39(6), 729-736. doi:10.2224/sbp.2011.39.6.729

Green, S. B., \& Salkind, N. J. (2011). Using SPSS for Windows and Macintosh: Analyzing and understanding data (6th ed.). Upper Saddle Rock, N.J.: Pearson Prentice Hall.

Greenberger, E., Josselson, R., Knerr, C., \& Knerr, B. (1974). The measurement and structure of psychosocial maturity. Journal of Youth and Adolescence, 4, 127-143. doi:10.1007/BF01537437

Groth, N. J. (1972). Achievement of autonomy and other developmental tasks in bright and average adolescents. Gifted Child Quarterly, 17, 64-67. doi:10.1177/001698627201600404

Häfner, A., \& Stock, A. (2010).Time management training and perceived control of time at work. The Journal of Psychology, 144(5), 429-447. doi:10.1080/00223980.2010.496647

Hall, E. T. (1983). The dance of life: The other dimension of life. Garden City, N.Y.: Anchor Press.

Hamilton, J. M., Kives, K. D., Micevski, V., \& Grace, S. L. (2003). Time perspective and health-promoting behavior in a cardiac rehabilitation population. Behavioral Medicine, 28, 132-139. doi:10.1080/08964280309596051

Haney, C., \& Zimbardo, P. G. (2009). Persistent dispositionalism in interactionist clothing: Fundamental attribution error in explaining prison abuse. Personality and Social Psychology Bulletin, 35, 807-814. doi:10.1177/0146167208322864

Hannah, S. (2010). Factors influencing a learner's decision to drop-out or persist in higher education distance learning. Retrieved from http://www.westga.edu/ distance/ojdla/winter134/street134.html

Harber, K. D., Zimbardo, P. G., \& Boyd, J. N. (2003). Participant self-selection biases as a function of individual differences in time perspective. Basic and Applied Social Psychology, 25, 255-264. doi:10.1207/S15324834BASP2503_08

Hicks, J., Davis, W., Trent, J. \& King, L. (2011). Positive affect, meaning in life, and future time perspective: An application of socioemotional selectivity theory. Psychology and Aging, 1(27), 181-189. doi: 10.1037/a0023965

Heidegger, M. (1927). Being and time. Halle, Germany: Niemeyer.

Hilpert, J. C., Husman, J., Stump, G. S., Kim, W., Chung, W. T., \& Duggan, M. A. (2012). Examining students" future time perspective: Pathways to knowledge building1. Japanese Psychological Research, 54(3), 229-240. doi:10.1111/j.1468-5884.2012.00525.x

Hodgins, D. C., \& Engel, A. (2002). Future time perspective in pathological gamblers. Journal of Nervous and Mental Disease, 190, 775-780. doi:10.1097/00005053-200211000-00008 
Holman, E. A., \& Silver, R. C. (1998). Getting "stuck" in the past: Temporal orientation and coping with trauma. Journal of Personality and Social Psychology, 74(5), 1146. doi:10.1037//0022-3514.74.5.1146

Holman, E. A., \& Zimbardo, P. G. (2009). The social language of time: The time perspective-social network connection. Basic and Applied Social Psychology, 31, 136-147. doi:10.1080/01973530902880415

Honora, D. (2002). The relationship of gender and achievement to future outlook among African American adolescents. Adolescence, 37, 301-316. Retrieved from http://www.ncbi.nlm.nih.gov/pubmed/12144161

Horstmanshof, L., \& Zimitat, C. (2007). Future time orientation predicts academic engagement among first-year university students. British Journal of Educational Psychology, 77, 703-718. doi:10.1348/000709906X160778

Husserl, E. (1964). Phenomenology of internal time consciousness. (M. Heidegger, Ed.; J. Churchill, Trans.). Bloomington: Indiana University Press.

James, W. (1890). The principles of psychology. Cambridge, M.A.: Harvard UP.

Janssen, M. A., Holahan, R., Lee, A., \& Ostrom, E. (2010). Lab experiments for the study of social-ecological systems. Science, 328, 613-617. doi:10.1126/science.1183532

Jessup, R. K., \& O’Doherty, J. P. (2011). Human dorsal striatal activity during choice discriminates reinforcement learning behavior from the gambler's fallacy. The Journal of Neuroscience, 31, 6296-6304. Retrieved from http://www. ncbi.nlm.nih.gov/pubmed/21525269

Jovanovski, D., \& Bassili, J. N. (2007). The relationship between morningness-eveningness preference and online learning. Biological Rhythm Research, 38, 355-365. doi:10.1080/09291010600950149

Kairys, A. (2010). Correlations between time perspectives and personality traits in different age groups. Bridge-/-Tiltai, 51(2), 159-172. Retrieved from https://erms.tourolib.org/url/http://search.ebscohost.com/login.aspx?direct=true\&db=a9h\&AN=531 74660\& site $=$ ehost-live

Kant, I. (1965). Critique of pure reason (N. Smith, Trans.). New York, N.Y.: Dover.

Kaufman, L., \& Rousseeuw, P. J. (2009). Finding groups in data: An introduction to cluster analysis (Vol. 344). New York: John Wiley \& Sons.

Keough, K. A., Zimbardo, P. G., \& Boyd, J. N. (1999). Who's smoking, drinking, and using drugs? Time perspective as a predictor of substance use. Basic and Applied Social Psychology, 21, 149-164. doi:10.1207/S15324834BA210207

King, D., \& Chen, S. (2009). Metrics for social games. Proceedings of the Social Games Summit, San Francisco, USA, 2009.

Klapproth, F. (2011). Stable and variable characteristics of the time perspective in humans. KronoScope, 11(1-2), 41-59. doi: $10.1163 / 156852411 X 595251$

Kruger, D. J., Reischl, T., \& Zimmerman, M. A. (2008). Time perspective as a mechanism for functional developmental adaptation. Journal of Social, Evolutionary, and Cultural Psychology, 2(1), 1-22. Retrieved from http://www-personal. umich.edu/ kruger/Kruger_et_al._Time_Perspective_LHT.pdf

Kvavik, R. B., \& Caruso, J. B. (2005). ECAR study of students and information technology, 2005: Convenience, connection, control, and learning. Boulder, C.O.: EducauseCenter for Applied Research. Retrieved from http://www.educause.edu/ LibraryDetailPage/666?ID=ERS0506

Laghi, F., D’Alessio, M., Pallini, S., \& Baiocco, R. (2009). Attachment representations and time perspective in adolescence. Social Indicators Research, 90, 181-194. doi:10.1007/s11205-008-9249-0

Lamm, H., Schmitdt, R., \& Trommsdorff, G. (1976). Sex and social class as determinants of future-orientation (time perspective) in adolescents. Journal of Personality and Social Psychology, 34, 317-326. doi:10.1037/0022-3514.34.3.317

Lee, A., \& Ji, L. J. (2013). Moving away from a bad past and toward a good future: Feelings influence the metaphorical understanding of time. Journal of Experimental Psychology: General. Advanced online publication. doi:10.1037/a0032233

Lens, W., Paixao, M. P., Herrera, D., \& Grobler, A. (2012). Future time perspective as a motivational variable: Content and extension of future goals affect the quantity and quality of motivation. Japanese Psychological Research, 54(3), 321-333. doi:10.1111/j.1468-5884.2012.00520.x

Levin, I., \& Zakay, D. (Eds.). (1989). Time and human cognition, advances in psychology series \# 59. Amsterdam, North-Holland: Elsevier Science.

Levy, K. N., Ellison, W. D., Scott, L. N., \& Bernecker, S. L. (2011). Attachment style. Journal of Clinical Psychology, 67, 193-203. doi:10.1002/jclp.20756

Lewin, K. (1951). Field theory in the social sciences: Selected theoretical papers. New York, N.Y.: Harper.

Lewin, T. (2012, May 2). Clash of the titans! (for online academics). The New York Times. Retrieved from http://www.msnbc.msn.com/id/47269703/ns/us_newsthe_new_york_times/t/clash-titans-online-academics/ 
Liaw, S. S., Huang, H. M., \& Chen, G. D. (2007). Surveying instructor and learner attitudes toward e-learning. Computers \& Education, 49, 1066-1080. doi: 10.1016/j.compedu.2006.01.001

Licht, M. H. (1995). Multiple regression and correlation. In L. A. Grimm, \& P. R. Yarnold (Eds.), Reading and understanding multivariate statistics (pp. 19-64). Washington, D.C.: American Psychological Association.

Lohr, J. M. (2011). What is (and what is not) the meaning of evidence-based psychosocial intervention? Clinical Psychology: Science and Practice, 18, 100-104. doi:10.1111/j.1468-2850.2011.01240.x

Long, L., Dubois, C., \& Faley, R. (2009). A case study analysis of factors that influence attrition rates in voluntary online training programs. International Journal on E-Learning, 8, 347-359. Retrieved from http://www.editlib.org/p/26285

Lundman, B., Strandberg, G., Eisemann, M., Gustafson, Y., \& Brulin, C. (2007). Psychometric properties of the Swedish version of the Resilience Scale. Scandinavian Journal of Caring Sciences, 21, 229-237. doi:10.1111/j.1471-6712.2007.00461.x

Luyckx, K., Lens, W., Smits, I., \& Goossens, L. (2010). Time perspective and identity formation: Short-term longitudinal dynamics in college students. International Journal of Behavioral Development, 34, 238-247. doi:10.1177/0165025409350957

Lysaght, R., Donnelly, C., \& Villeneuve, M., (2009). Factors predicting applicant outcomes in occupational therapy education. The Canadian Journal of Occupational Therapy, 76(1), 38-47. doi:10.1177/000841740907600110

MacCann, C., Fogarty, G. J., Zeidner, M., \& Roberts, R. D. (2011). Coping mediates the relationship between emotional intelligence (EI) and academic achievement. Contemporary Educational Psychology, 36, 60-70. doi:10.1016/j.cedpsych.2010.11.002

Matthews, W. J., Stewart, N., \& Wearden, J. H. (2011). Stimulus intensity and the perception of duration. Journal of Experimental Psychology: Human Perception and Performance, 37, 303-313. doi:10.1037/a0019961

McWilliams, L. A., Murphy, P. D. J., \& Bailey, S. J. (2010). Associations between adult attachment dimensions and attitudes toward pain behaviour. Pain Research \& Management: The Journal of the Canadian Pain Society, 15, 378-384. Retrieved from http://www.pulsus.com/journals/abstract.jsp?origPg=abstract.jsp\&sCurrPg=abstract\&jnlKy=7\&atlKy=9886\&isuKy= 956\&isArt=t\&fromfold $=$ Current $\% 20$ Issue \&\&HCtype $=$ Physician

Mello, Z. R., Worrell, F. C., \& Andretta, J. R. (2009). Variation in how frequently adolescents think about the past, the present, and the future in relation to academic achievement. DiskursKindheits- Und Jugendforschung, 4(2), 173-183. Retrieved from $\mathrm{http} / /$ web.ebscohost.com/ehost/detail?vid=3\&hid=21\&sid=e51570ea-341a-499097c020446a3b659b\%40sessionmgr10\&bdat

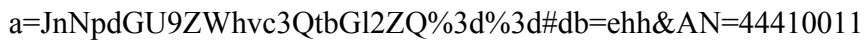

Milfont, T. L., Andrade, P. R., Bela, R. P., \& Pessoa, V. S. (2008). Testing Zimbardo Time Perspective Inventory in a Brazilian sample. Interamerican Journal of Psychology, 42(1), 49-58. Retrieved from http://www.psicorip.org/Resumos/PerP/RIP/ RIP041a5/RIP04206.pdf

Moore, D. S. (2007). The basic practice of statistics (4th ed.). New York, N.Y.: W. H. Freeman.

Moore, M. G., \& Kearsley, G. (2012). Distance education: A systems view of online learning (3rd ed.). Belmont, C.A.: Wadsworth Cengage Learning.

Monahan, K. C., Steinberg, L., Cauffman, E., \& Mulvey, E. P. (2010). Trajectories of antisocial behavior and psychosocial maturity from adolescence to young adulthood. Developmental Psychology, 45, 1654-1666. doi:10.1037/a0015862

Moscovici, S. (1984). The phenomenon of social representations (S. Rabinovitch, Trans.). In R. Farr, \& S. Moscovici (Eds.), Social representations (pp. 3-69) Cambridge, England: Cambridge University Press.

National Center for Education Statistics, U.S. Department of Education. (2007). Distance education at degree-granting postsecondary institutions: 2006-2007. Washington, D.C.

Neves, A. A. L. (2009). Are delay discounting, probability discounting, time perception, and time perspective related? A cross-cultural study among Latino and White American students (Doctoral dissertation). Retrieved from http://digitalcommons.usu.edu/etd/cgi/viewcontent.cgi?article=1969\&context=etd

Nichols, A. J., \& Levy, Y. (2009). Empirical assessment of college student- athletes' persistence in e-learning courses: A case study of a National Association of Intercollegiate Athletes (NAIA) instruction. Internet and Higher Education, 12(1), 14-25. doi: 10.1016/j.iheduc.2008.10.003

Nishi, D., Uehara, R., Kondo, M., \& Matsuoka, Y. (2010). Reliability and validity of the Japanese version of the Resilience Scale and its short version. Biomedical Center Research Notes, 310(3), 1-6. doi:10.1186/1756-0500-3-310

Noulhiane, M., Mella, N., Samson, S., Ragot, R., \& Pouthas, V. (2007). How emotional auditory stimuli modulate time perception. Emotion, 7, 697-704. doi:10.1037/1528-3542.7.4.697

Núñez, J. C., Suárez, N., Cerezo, R., González-Pienda, J., Rosário, P., Mourão, R., \& Valle, A. (2013). Homework and academic achievement across Spanish compulsory education. Educational Psychology, 34, 1-21. doi:10.1080/01443410.2013.817537 


\section{REFERENCES}

Nuttin, J. (1964). The future time perspective in human motivation and learning. Acta Psychologica, 23, 60-83. doi:10.1016/0001-6918(64)90075-7

Nuttin, J., \& Lens, W. (1985). Future time perspective and motivation: Theory and research method. Leuven \& Hillsdale, N.J.: Leuven University Press and Erlbaum.

Olley, B. O. (2010). The role of insecure and fearful attachment styles in sexual risk behaviors among HIV-positive women attending a support group in Ibadan, Nigeria. Gender \& Behaviour, 8, 3389-3406. Retrieved from $\mathrm{http}: / / \mathrm{www}$.readperiodicals.com/201012/2187713451.html

Orenstein, R. (1975). On the experience of time. Baltimore: Penguin.

Ortega, L., \& Lopez, F. (2008). Effects of visual flicker on subjective time in a temporal bisection task. Behavioral Process, 78, 380-386. doi:10.1016/j.beproc.2008.02.004

Osborne, J. W., \& Waters, E. (2002). Four assumptions of multiple regression that researchers should always test. Practical Assessment, Research, and Evaluation, 8(2), 1-5. Retrieved from http://www-psychology.concordia.ca/fac/kline/601/ osborne.pdf

Oyserman, D., \& Markus, H. (1990a). Possible selves in balance: Implications for delinquency. Journal of Social Issues, 46, 141-157. doi:10.1111/j.1540-4560.1990.tb01927.x

Oyserman, D., \& Markus, H. (1990b). Possible selves and delinquency. Journal of Personality and Social Psychology, 59, 112-125. doi:10.1037/0022-3514.59.1.112

Padawer, E. A., Jacobs-Lawson, J. M., Hershey, D. A., \& Thomas, D. G. (2007). Demographic indicators as predictors of future time perspective. Current Psychology, 26, 102-108. doi:10.1007/s12144-007-9008-4

Pappano, L. (2012, November 12). Year of the MOOC. The New York Times. Retrieved from http://www.nytimes. com/2012/11/04/education/edlife/massive-open-onlinecourses-are-multiplying-at-a-rapid-pace.html?pagewanted=all\&_r=0

Parsad, B., \& Lewis, L. (2008). Distance education at degree-granting post-secondary institutions: 2006-2007. U.S. Department of Education, Institute of Education Sciences, National Center for Educational Statistics. Retrieved from http://nces.ed.gov/pubsearch/index.asp?HasSearched=18.searchcat $2=$ subjectindex $\&$ L1 $=166 \& \mathrm{~L} 2=0$

Pascual-Leone, J., \& Johnson, J. (2011). A developmental theory of mental attention. In P. Barrouillet, \& V. Guillard (Eds.), Cognitive development and working memory: A dialogue between neo-Piagetian theories and cognitive approaches ( $\mathrm{pp}$. 13-42). New York, N.Y.: Psychological Press.

Patterson, B., \& McFadden, C. (2009). Attrition in online and campus degree programs. Online Journal of Distance Learning Administration, 12(11), 1-10. Retrieved from http://www.educause.edu/Resources/AttritioninOnlineandCampusDegr/177047

Peetsma, T. (1994). Future time perspective as a predictor of school engagement. Journal of Education Research, 19, 331-342. doi:10.1016/j.learninstruc.2010.08.001

Peetsma, T., \& van der Veen, I. (2011). Relations between the development of future time perspective in three life domains, investments in learning, and academic achievement. Learning and Instruction, 21(3), 481-494. doi:10.1016/j.learninstruc.2010.08.001

Peetsma, T., Schuitema, J., \& van der Veen, I. (2012). A longitudinal study in time perspectives: Relations with academic delay gratification and learning environment. Japanese Psychological Research, 54(3), 241-252. doi:10.1111/j.1468-5884.2012.00526.x

Power, M., \& Gould-Morven, A. (2011). Head of gold, feet of clay: The online learning paradox. International Review of Research in Open and Distance Learning, 12(2), 19-38. Retrieved from http://www.irrodl.org/index.php/irrodl/article/view/ 916/1785

Pratt, T. C., Cullen, F. T., Sellers, C. S., Winfree, L. T., Madensen, T. D., Daigle, L. H, ... Gau, J. M. (2010). Empirical status of social learning theory: A meta-analysis. Justice Quarterly, 27, 765-802. doi:10.1080/07418820903379610

Ramos, J. A. (2011). A comparison of perceived stress levels and coping styles of non-traditional graduate students in distance learning versus on-campus programs. Contemporary Educational Technology, 2(4), 282-293. Retrieved from: http://scholar.googleusercontent.com/scholar?q=cache:URVCj2qvj_EJ:scholar.google.com/\&hl=en\&as_sdt=1,33

Ryan, R. M., \& Deci, E. L. (2011). A self-determination theory perspective on social, institutional, cultural, and economic supports for autonomy and their importance for well-being. In A. Fave (Series Ed.), Human autonomy in cross-cultural context: Vol. 1. Perspectives on the psychology of agency, freedom, and well-being (pp. 45-64). New York, N.Y.: Springer.

Sarwar, M., Inamullah, H., Khan, N., \& Anwar, N. (2010). Resilience and academic achievement of male and female secondary level students in Pakistan. Journal of College Teaching \& Learning, 7(8), 19-24. Retrieved from http://www.mendeley.com/research/resilience-academic-achievement-male-female-secondary-level-students-pakistan-1/ 
Schielzeth, H. (2010). Simple means to improve the interpretability of regression coefficients. Methods in Ecology and Evolution, 1, 103-113. doi:10.1111/j.2041-210X.2010.00012.x

Schneider, J. A. (2010). From Freud's dream-work to Bion's work of dreaming: The changing conception of dreaming in psychoanalytic theory. Journal of Psychoanalysis, 91, 521-540. doi:10.1111/j.1745-8315.2010.00263.x

Seginer, R. (2009). Future orientation: Developmental and ecological perspectives. New York: Springer.

Seginer, R., \& Shoyer, S. (2012). How mothers affect adolescents' future orientation: A two-source analysis. Japanese Psychological Research, 54(3), 310-320. doi:10.1111/j.1468-5884.2012.00522.x

Severance, C. (2012). Teaching the world: Daphne Koller and Coursera. Computer, 45(8), 8-9. doi:10.1109/MC.2012.278

Selingo, J. (2012). As elite colleges invite the world online, questions remain on their business plans. The Chronicle of Higher Education. Retrieved from http://chronicle.com/blogs/next/2012/05/19/as-elite-colleges-open-tothe-world-online-questionsremain-on-business-plan/

Shell, D. F., \& Husman, J. (2001). The multivariate dimensionality of personal control and future time perspective beliefs in achievement and self-regulation. Contemporary Educational Psychology, 26, 481-506. doi:10.1006/ceps.2000.1073

Shirai, T., Nakamura, T., \& Katsuma, K. (2012). Time orientation and identity formation: Long-term longitudinal dynamics in emerging adulthood1. Japanese Psychological Research, 54(3), 274-284. doi:10.1111/j.1468-5884.2012.00528.x

Silva, M. N., Vieira, P. N., Coutinho, S. R., Matos, M. G., Sardinha, L. B., \& Teixeira, P. J. (2010). Using self-determination theory to promote physical activity and weight control: A randomized controlled trial in women. Journal of Behavioral Medicine, 33, 110-122. doi:10.1007/s10865-009-9239-y

Skinner, B. F. (1987). Upon further reflection. Englewood Cliffs, N.J.: Prentice-Hall.

Statistics, S. P. S. S. (2011). Version 19.0. Chicago: SPSS Inc.

Stevens, J. P. (2002). Applied multivariate statistics for the social sciences (4th ed.). Mahwah, N.J.: Lawrence Erlbaum Associates.

Stolarski, M., Bitner, J., \& Zimbardo, P. G. (2011). Time perspective, emotional intelligence and discounting of delayed awards. Time \& Society, 20(3), 346-363. doi:10.1177/0961463X11414296

Strathman, A., Gleicher, F., Boninger, D., \& Edwards, C. (1994). The consideration of future consequences: Weighing immediate and distant outcomes of behavior. Journal of Personality and Social Psychology, 66, 742-752. doi:10.1037/0022-3514.66.4.742

Street, H. D. (2010). Factors influencing a learner's decision to drop-out or persist in higher education distance learning. Online Journal of Distance Learning Administration, 13(4). Retrieved from http://www.editlib.org/p/52605

Surveymonkey (Apparatus and software). Palo Alto, C.A.: SurveyMonkey.com, LLC. Retrieved from http://www.surveymonkey.com

Swain, M., Kinnear, P., \& Steinman, L. (2011). Sociocultural theory in second language education: An introduction through narratives. Bristol, U.K.: Multilingual Matters.

Swart, A. J., Lombard, K., \& de Jager, H. (2010). Exploring the relationship between time management skills and the academic achievement of African engineering students-A case study. European Journal of Engineering Education, 35(1), 79-89. doi:10.1080/03043790903480316

Taatgen, N. A., van Rijn, H., \& Anderson, J. (2007). An integrated theory of prospective time interval estimation: The role of cognition, attention, and learning. Psychological Review, 114, 577-598. doi:10.1037/0033-295X.114.3.577

Teddlie, C., \& Tashakkori, A. (2009). Foundations of mixed methods research: Integrating quantitative and qualitative approaches in the social and behavioral sciences (Kindle Edition). Thousand Oaks, C.A.: SAGE Publications.

Thompson, R. A. (2008). Early attachment and later developments. In J. Cassidy, \& P. Shaver (Eds.), Handbook of attachment: Theory, research and clinical applications (pp. 348-365). New York, N.Y.: Guilford.

Thompson, M. E., Ford, R., \& Webster, A. (2011). Effectiveness of interactive, online games in learning neuroscience and students' perception of the games as learning tools: A pre-experimental study. Journal of Allied Health, 40(3), 150-155. Retrieved from http://www.asahp.org/pdf/Thompson\%20JAH_Fall11-5.pdf

Tokita, M., \& Ishiguchi, A. (2011). Temporal information affects the performance of numerosity discrimination: Behavioral evidence for a shared system for numerosity and temporal processing. Psychonomic Bulletin and Review, 18, 550-556. doi:10.3758/s13423-011-0072-2

Tranmer, M., \& Elliot, M. (2008). Multiple linear regression: Cathie Marsh Centre for Census and Survey Research (CCSR). Retrieved from http://www.ccsr.ac.uk/publications/teaching/mlr.pdf

Trochim, W. M., \& Donnelly, J. P. (2008). Research methods knowledge base. Atomic Dog/Cengage Learning. 
University of Phoenix-online campus- Phoenix, Arizona: details, students, expenses. (2012). Retrieved from http://colleges.find thebest.com/1/5097/Universityof-Phoenix-Online-Campus.

Urien, B. (2007). Selfless time. Time \& Society, 16, 367-386. doi:10.1177/0961463X07080273

Utsey, S. O., Hook, J. N., Fischer, N., \& Belvet, B. (2008). Cultural orientation, ego resilience, and optimism as predictors of subjective well-being in African Americans. Journal of Positive Psychology, 3, 202-210. doi:10.1080/17439760801999610

Uzma, A. (2007). Relationship between academic resilience and academic achievement of postgraduate students of University of Sargodha. Journal of College Teaching Learning, 7(8), 19-24. Retrieved from http://www.cluteinstitute.com

Van der Meer, J., Jansen, E., \& Torenbeek, M. (2010). "It's almost a mindset that teachers need to change": First year students need to be inducted into time management. Studies in Higher Education, 35(7), 777-791. doi:1080/03075070903383211

Varney, J. J. (2010). The role of dissertation self-efficacy in increasing dissertation completion: Sources, effects and viability of a new self-efficacy construct. College Student Journal, 44, 932-947. Retrieved from http://findarticles.com/p/articles/mi_m0F CR/is_4_44/ai_n56882822/

Vowinckel, J. C. (2012). Balanced time perspectives and mindfulness. Retrieved from: http://essay.utwente.nl/62422/1/ Vowinckel,_J.C._-_s0160067_(verslag).pdf

Wagnild, G. M. (2011). The Resilience Scale user's guide. Worden, M.T.: ResilienceCenter.

Wagnild, G. M., \& Collins, J. A. (2009). Assessing resilience. Journal of Psychosocial Nursing and Mental Health Services, 47(12), 28-33. doi:10.3928/02793695-20091103-01

Wagnild, G. M., \& Young, H. (1993). Development and psychometric evaluation of the Resilience Scale. Journal of Nursing Measurement, 1(2), 165-178. Retrieved from http://www.sapibg.org/attachments/article/1054/wagnild_1993_resilience_scale 2.pdf

Wagnild, G. M., \& Young, H. (2009). The 14-Item Resilience Scale (RS-14).Wordern, M.T.: Resilience Center.

Watson, J. (1913). Psychology as the behaviorist views it. Psychological Review, 20, 158-177. doi:10.1037/h0074428

Webster, J. D. (2011). A new measure of time perspective: initial psychometric findings for the Balanced Time Perspective Scale (BTPS). Canadian Journal of Behavioural Science, 43(2), 111-118. doi: 10.1037/a0022801

Wentzel, K., Battle, A., Russell, S., \& Looney, L. (2010). Social supports from teachers and peers as predictors of academic and social motivation. Contemporary Educational Psychology, 35(3), 193-202. doi:10.1016/j.cedpsych.2010.03.002

Wills, T. A., Sandy, J. M., \& Yaeger, A. M. (2001). Time perspective and early-onset substance use: A model based on stress-coping theory. Psychology of Addictive Behaviors, 15, 118-125. doi:10.1037/0893-164X.15.2.118

Winnicott, D. W. (1953). Transitional objects and transitional phenomena-A study of the first not-me possession 1. International Journal of Psycho-Analysis, 34, 89-97. Retrieved from http://icpla.edu/wp-content/uploads/2013/02/Winnicott-D.Transitional-Objects-and-Transitional-Phenomena.pdf

Wittmann, M. (2009). The inner experience of time. Philosophical Transactions Royal Society B, 36(1525), $1955-1967$. doi:10.1098/rstb.2009.0003

Wittmann, M., \& Paulus, M. P. (2008). Decision making, impulsivity and time perception. Trends in Cognitive Science, 12(1), 7-12. doi:10.1016/j.tics.2007.10.004

Wittmann, M., \& van Wassenhove, V. (2009). The experience of time: Neural mechanisms and the interplay of emotion, cognition and embodiment. Philosophical Transactions of the Royal Society Biological Sciences, 364, 1809-1813. doi:10.1098/rstb.2009.0025

Worrel, F. C., \& Mello, Z. R. (2007). The reliability and validity of Zimbardo Time Perspective Inventory scores in academically talented adolescents. Educational and Psychological Measurement, 67, 487-504. doi:10.1177/0013164406296985

Worrell, F. C., \& Mello, Z. R. (2009). Convergent and discriminate validity of time attitude scores on the Adolescent Time Perspective Inventory. Diskurs Kindheits- und Judgendforschung Heft, 2, 185-196. Retrieved from http://budrich-journals.de/ index.php/diskurs/article/viewArticle/79

Xie, X., Liu, H., \& Gan, Y. (2010). Belief in a just world when encountering the 5/12 Wenchuan earthquake. Environment and Behavior, 43, 566-586. doi:10.1177/0013916510363535

Yazici, H., Seyis, S., \& Altun, F. (2011). Emotional intelligence and self-efficacy beliefs as predictors of academic achievement among high school students. Procedia-Social and Behavioral Sciences, 15, 2319-2323. doi:10.1016/j.sbspro.2011.04.100

Yoo, J., Slack, K. S., \& Holl, J. L. (2010). The impact of health- promoting behaviors on low-income children's health: A risk and resilience perspective. Health \& Social Work, 35, 133-143. doi:10.1002/nur.20004

Zakay, D. (1989). Subjective time and attentional resource allocation: An integrated model of time estimation. In I. Levin, \& D. Zakay (Eds.), Time and human cognition: A life span prospective (pp. 365-398). Amsterdam, North-Holland: Elsevier Science. 
Zaleski, Z. (1996). Future anxiety: Concept measurement and preliminary research. Personality and Individual Differences, 21, 165-174. doi:10.1016/0191-8869(96)00070-0

Zaleski, Z. (1987). Behavioural effects of self-set goals for different time ranges. International Journal of Psychology, 22, 17-38. doi:10.1080/00207598708246765

Zampetakis, L. A., Bouranta, N., \& Moustakis, V. S. (2010). On the relationship between individual creativity and time management. Thinking Skills and Creativity, 5, 23-32. doi: 10.1016/j.tsc.2009.12.001

Zandieh, M., \& Habibi, M. (2011). The role of distance education in improving adult education. Journal of American Science, 7 , 327-331. Retrieved from http://www.jofamericanscience.org/journals/am-sci/am0706/58_5607am0706_327_331.pdf

Zautra, A. J., Hall, J. S., \& Murray, K. E. (2010). Resilience: A new definition of health for people and communities. In J. Reich, A., Zautra, \& J. Hall (Eds.), Handbook of adult resilience (pp. 3-34). New York, N.Y.: Guilford.

Zembylas, M. (2008). The politics of trauma in education. New York: Palgrave Macmillan.

Zhang, J. W., \& Howell, R. T. (2011). Do time perspectives predict unique variance in life satisfaction beyond personality traits? Personality and Individual Differences, 50(8), 1261-1266. doi:10.1016/j.paid.2011.02.021

Zhang, J. W., Howell, R. T., \& Stolarski, M. (2013). Comparing three methods to measure a balanced time perspective: The relationship between a balanced time perspective and subjective well-being. Journal of Happiness Studies, 14(1), 1-16. doi: $10.1234 / 12345678$

Zimbardo, P. G. (2007). The Lucifer effect. New York, N.Y.: Random House.

Zimbardo, P. G., \& Boyd, J. N. (1999). Putting time in perspective: A valid, reliable individual-differences metric. Journal of Personality and Social Psychology, 77, 1271-1288. Retrieved from http://www.timeperspective.com/Articles/1999Putting TimeinPerspective.pdf

Zimbardo, P., \& Boyd, J. N. (2008). The time paradox: The new psychology of time that will change your life. New York, N.Y.: Free Press.

Zimbardo, P. G., Keough, K. A., \& Boyd, J. N. (1997). Present time perspective as a predictor of risky driving. Personality and Individual Differences, 23, 1007-1023. doi:10.1016/S0191-8869(97)00113-X

Zimbardo, P. G., Marshall, G., \& Maslach, C. (1971). Liberating behavior from time-bound controls: Expanding the present through hypnosis. Journal of Applied Social Psychology, 1, 305-323. doi:10.1111/j.1559-1816.1971.tb00369.x

Zuckerman, M. (1994). Behavioral expressions and biosocial bases of sensation seeking. New York: Cambridge University Press.

Zuckerman, M., Eysenck, S. B. G., \& Eysenck, H. J. (1978). Sensation seeking in England and America: Cross-cultural, age and sex comparisons. Journal of Consulting and Clinical Psychology, 39, 308-321. doi:10.1037/0022-006X.46.1.139 


\section{Acknowledgements}

I would like to thank the following individuals for their support in the publication of this volume. In alphabetical order, they are: Dr. Andrew Carpenter, Peter Labarbera, Dr. Marilyn Maxwell, Dr. Robert Mollinger, and Dr. Philip Zimbardo. In addition, thank you to Freud, Lincoln, and Poe. 